DEPARTAMENTO DE MATEMÁTICA APLICADA A LA INGENIERÍA AEROESPACIAL

ESCUELA TÉCNICA SUPERIOR DE INGENIERÍA AERONÁUTICA Y DEL ESPACIO

Doctoral Thesis

\title{
Adaptation Strategies for Discontinuous Galerkin Spectral Element Methods by means of Truncation Error Estimations
}

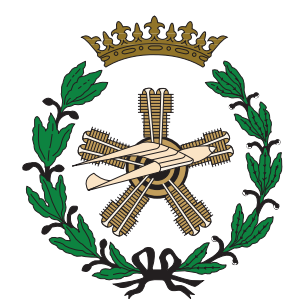 \\ by \\ Moritz Kompenhans \\ Computer and Aeronautical Engineer \\ Advisors \\ Esteban Ferrer Vaccarezza \\ Ph.D. in Aeronautical Engineering \\ Eusebio Valero Sánchez \\ Ph.D. in Aeronautical Engineering
}

MAdRID, MARCH 2016 



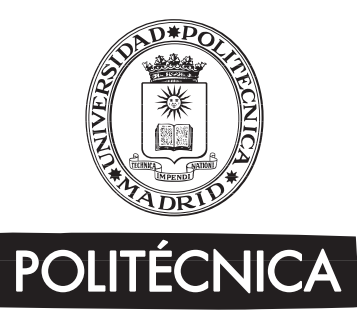

El tribunal nombrado por el Sr. Rector Magnífico de la Universidad Politécnica de Madrid, el día de de

Presidente: JUAN ANTONIO HERNANDEZ RAMOS

Vocal: FRANÇOIS FRAYSSE

Vocal: JORGE PONSÍN ROCA

Vocal: ANTONIO PASCAU BENITO

Secretario: GONZALO RUBIO CALZADO

Suplente: DANIEL RODRÍGUEZ ÁLVAREZ

Suplente: MARIO MARTIN BURGOS

Realizado el acto de defensa y lectura de la Tesis el día de de en la E.T.S.I./Facultad Aeronáuticos

Calificación:

EL PRESIDENTE

LOS VOCALES

EL SECRETARIO 



\title{
Adaptation Strategies for Discontinuous Galerkin Spectral Element Methods by means of Truncation Error Estimations
}

\begin{abstract}
In this work a p-adaptation (modification of the polynomial order) strategy based on the minimization of the truncation error is developed for high order discontinuous Galerkin methods.

The truncation error is approximated by means of a truncation error estimation procedure and enables the identification of mesh regions that require adaptation. Three truncation error estimation approaches are developed and termed a posteriori, quasi- $a$ priori and quasi- $a$ priori corrected. Fine solutions, which are obtained by enriching the polynomial order, are required to solve the numerical problem with adequate accuracy. For the three truncation error estimation methods the former needs time converged solutions, while the last two rely on non-converged solutions, which lead to faster computations.

Based on these truncation error estimation methods, algorithms for mesh adaptation were designed and tested. Firstly, an isotropic adaptation approach is presented, which leads to equally distributed polynomial orders in different coordinate directions. This first implementation is improved by incorporating a method to extrapolate the truncation error. This results in a significant reduction of computational cost. Secondly, the employed high order method permits the spatial decoupling of the estimated errors and enables anisotropic p-adaptation. The incorporation of anisotropic features leads to meshes with different polynomial orders in the different coordinate directions such that flow-features related to the geometry are resolved in a better manner. These adaptations result in a significant reduction of degrees of freedom and computational cost, while the amount of improvement depends on the test-case. Finally, this anisotropic approach is extended by using error extrapolation which leads to an even higher reduction in computational cost.

These strategies are verified and compared in terms of accuracy and computational cost for the Euler and the compressible Navier-Stokes equations. The main result is that the two quasi-a priori methods achieve a significant reduction in computational cost when compared to a uniform polynomial enrichment. Namely, for a viscous boundary layer flow, we obtain a speedup of a factor of 6.6 and 7.6 for the quasi- $a$ priori and quasi- $a$ priori corrected approaches, respectively.

Keywords: discontinuous Galerkin, spectral methods, $\tau$-estimation, truncation error, a posteriori error estimation, compressible Navier Stokes, incompressible Navier Stokes, hp-adaptation
\end{abstract}





\section{Estrategias de adaptación para Discontinuous Galerkin Spectral Element Method mediante estimaciones del error de truncación}

\section{Resumen}

En este trabajo se ha desarrollado una estrategia de adaptación-p (modificación del orden polinómico) para métodos Galerkin discontinuo de alto orden basada en la minimización del error de truncación.

El error de truncación se estima utilizando el método tau-estimation. El estimador permite la identificación de zonas de la malla que requieren adaptación. Se distinguen tres técnicas de estimación: a posteriori, quasi a priori y quasi a priori con correción. Todas las estrategias requieren una solución obtenida en una malla fina, la cual es obtenida aumentando de manera uniforme el orden polinómico. Sin embargo, mientras que el primero requiere que esta solución esté convergida temporalmente, el resto utiliza soluciones no convergidas, lo que se traduce en un menor coste computacional.

En este trabajo se han diseñado y probado algoritmos de adaptación de malla basados en métodos tau-estimation. En primer lugar, se presenta un algoritmo de adaptacin isótropo, que conduce a discretizaciones con el mismo orden polinómico en todas las direcciones espaciales. Esta primera implementación se mejora incluyendo un método para extrapolar el error de truncación. Esto resulta en una reducción significativa del coste computacional. En segundo lugar, el método de alto orden permite el desacoplamiento espacial de los errores estimados, permitiendo la adaptación anisotropica. Las mallas obtenidas mediante esta técnica tienen distintos órdenes polinómicos en cada una de las direcciones espaciales. La malla final tiene una distribución óptima de órdenes polinómicos, los cuales guardan relación con las características del flujo que, a su vez, depenen de la geometría. Estas técnicas de adaptación reducen de manera significativa los grados de libertad y el coste computacional. Por último, esta aproximación anisotrópica se extiende usando extrapolación del error de truncación, lo que conlleva un coste computational aún menor.

Las estrategias se verifican y se comparan en téminors de precisión y coste computacional utilizando las ecuaciones de Euler y Navier Stokes. Los dos métodos quasi a priori consiguen una reducción significativa del coste computacional en comparación con aumento uniforme del orden polinómico. En concreto, para una capa límite viscosa, obtenemos una mejora en tiempo de computación de 6.6 y 7.6 respectivamente, para las aproximaciones quasi-a priori y quasi-a priori con corrección.

Keywords: Galerkin discontinuo, métodos espectrales, Estimación tau, error de truncación, estimación del error a posteriori, Navier Stokes compresible , Navier Stokes incompresibles, adaptación hp 



\section{Acknowledgements}

The author would like to thank the European Commission for the financial support of the ANADE project (Advances in Numerical and Analytical tools for DEtached flow prediction) under grant contract PITN-GA-289428 and the collaboration with the NNATAC project (New Numerical and Analytical Tools for Aerodynamic flow Control) under grant agreement PIAP-GA-2012-324298. 


\section{Nomenclature}

\section{General}

$\begin{array}{ll}\rho & \text { Density } \\ p & \text { Pressure } \\ M & \text { Mach number } \\ R e & \text { Reynolds number } \\ C_{D} & \text { Drag coefficient } \\ C_{L} & \text { Lift coefficient }\end{array}$

\section{Discretisation}

$\Omega \quad$ Computational domain

$P_{k}^{(\alpha, \beta)}(x) \quad$ Jacobi Polynomials

$T_{k} \quad$ Chebyshev polynomial of degree $k$

$L_{k} \quad$ Legendre polynomial of degree $k$

$w_{i} \quad$ Quadrature weight at point $x_{i}$

$\xi, \eta \quad$ Computational coordinates on the unit square

$\xi_{i} \quad$ Nodal points

$l_{j}(\xi) \quad$ Lagrange interpolating polynomials

$u \quad$ Exact solution

$u^{N} \quad$ Approximate discrete solution

$\tilde{u} \quad$ Non time-converged solution

$\mathcal{R} \quad$ Partial differential operator

$\hat{\mathcal{R}} \quad$ Isolated partial differential operator

$\mathcal{R}^{N} \quad$ Discrete partial differential operator

$\hat{\mathcal{R}}^{N} \quad$ Isolated discrete partial differential operator

$\mathbf{f}^{*} \quad$ Approximation of the Riemann problem

$\mathcal{J}^{N}(u) \quad$ Functional output

\section{Error estimation}

$\begin{array}{ll}\varepsilon^{N} & \text { Discretisation error } \\ \varepsilon_{u}^{N} & \text { Interpolation error } \\ \varepsilon_{i t}^{N} & \text { Iteration error } \\ \tau^{N} & \text { Truncation error } \\ \hat{\tau}^{N} & \text { Isolated truncation error } \\ \tau_{P}^{N} & \text { Truncation error estimation (fine order } P \text { to coarse order } N \text { ) } \\ \tau_{\text {max }} & \text { Maximal allowed truncation error (for adaptation) }\end{array}$


$P \quad$ Polynomial order of fine solution

$N_{x} \quad$ Coarse polynomial order in x-direction

$N_{y} \quad$ Coarse polynomial order in y-direction

\section{Acronyms}

CFD Computational Fluid Dynamics

FVM Finite Volume Method

FEM Finite Element Method

DG Discontinous Galerkin

DGSEM Discontinous Galerkin Spectral Element Method

DoF Degrees of Freedom

LDoF Local Degrees of Freedom

AoA Angle of Attack 



\section{Contents}

1 Introduction $\quad 1$

1.1 Content . . . . . . . . . . . . . . . . . . . . 1

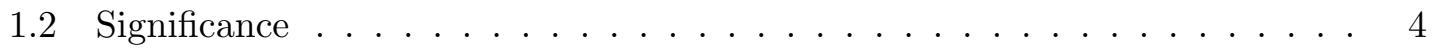

1.3 Publication and Outreach . . . . . . . . . . . . . . 6

1.4 Structure ........................... 6

2 Spectral Methods $\quad 9$

2.1 The Fourier Expansion . . . . . . . . . . . . . . . . . . . . . . . . 10

2.1.1 The Continuous Fourier Expansion . . . . . . . . . . . . . . . 10

2.1 .2 Discrete Truncated Series . . . . . . . . . . . . . . . . 11

2.1 .3 Orders of Convergence . . . . . . . . . . . . . . . . . 11

2.1.4 Fourier Series Convergence . . . . . . . . . . . . . . . . 13

2.1.5 Fourier Quadrature . . . . . . . . . . . . . . . . 15

2.1 .6 Fourier Interpolation . . . . . . . . . . . . . . . . . 16

2.1 .7 Fourier Interpolation Error _ . . . . . . . . . . . . . 16

2.1.8 Differentiation through Fourier Interpolation . . . . . . . . . . . . . . 18

2.2 Polynomial Basis Functions . . . . . . . . . . . . . . . . . . . . 18

2.2 .1 Polynomial Series . . . . . . . . . . . . . . . . . 20

2.2.2 Polynomial Quadrature . . . . . . . . . . . . . . . . 20

2.2 .3 Orthogonal Polynomial Interpolation . . . . . . . . . . . . . . . . 22

2.2.3.1 Polynomial Interpolation Error . . . . . . . . . . . . 23

2.2.3.2 Polynomial Interpolation based Differentiation . . . . . . 25

2.3 Approximation of PDE Solutions . . . . . . . . . . . . . . . . . 25

2.4 Discontinuous Galerkin Spectral Element Method . . . . . . . . . . . . 27

2.5 Implementation and Verification . . . . . . . . . . . . . . . . 30

3 Error Estimation $\quad 31$

3.1 Definitions of Errors . . . . . . . . . . . . . . . . . 33

3.2 DGSEM Error Analysis . . . . . . . . . . . . . . . . . . . . 34

3.2 .1 Discretization Error . . . . . . . . . . . . . . . . . 34

3.2 .2 Iteration Error . . . . . . . . . . . . . . . . . 35

3.2 .3 Truncation Error . . . . . . . . . . . . . . . . . 35

3.2.3.1 Isolated Truncation Error . . . . . . . . . . . . . . 35 
3.2.3.2 Error Dependencies . . . . . . . . . . . . . 36

3.2.3.3 Anisotropic Behaviour of the Truncation Error . . . . . . . 37

3.2.4 Truncation Error Limitations . . . . . . . . . . . . . . . . 37

3.2.5 Truncation Error and Functionals . . . . . . . . . . . 38

3.3 Error Estimation . . . . . . . . . . . . . . . . . . . . . . . . 39

4 Adaptation $\quad 43$

4.1 Isotropic Adaptation . . . . . . . . . . . . . . . . . . . . . . 44

4.1.1 Truncation / Isolated Truncation Error Adaptation Algorithm . . . 44

4.1 .2 Error Extrapolation . . . . . . . . . . . . . . . . . 46

4.1 .3 Discretisation Error _. . . . . . . . . . . . . . . . 48

4.1.3.1 Discretisation Error Estimation _. . . . . . . . . . . 49

4.1.3.2 Discretisation Error Adaptation Algorithm . . . . . . . . . 50

4.2 Anisotropic Adaptation . . . . . . . . . . . . . . . . . . . 51

4.2.1 Truncation / Isolated Truncation Error Adaptation Algorithm . . . 51

4.2 .2 Minimization of DoF . . . . . . . . . . . . . . . . 51

4.2.3 Error Extrapolation for minimal DoF . . . . . . . . . . . . 52

4.3 Quasi-a priori and quasi-a priori corrected $\tau$-estimation . . . . . . . . . 56

4.3 .1 Memory Requirements . . . . . . . . . . . . . . . . 56

4.3.2 Theoretical Computational Cost . . . . . . . . . . . . . . . 57

5 Numerical Results $\quad 59$

5.1 Manufactured Solution Test Case . . . . . . . . . . . . . . . . . . . . 60

5.1 .1 Estimated Error vs. Exact Error . . . . . . . . . . . . . . . 61

5.1.2 A posteriori and quasi- a priori Estimates . . . . . . . . . . . 62

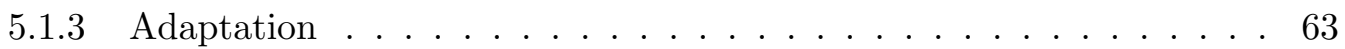

5.2 Inviscid NACA0012 Airfoil _ . . . . . . . . . . . . . . . . 67

5.3 Boundary Layer Test Case . . . . . . . . . . . . . . . . . . . . . . 72

5.3 .1 Adaptation . . . . . . . . . . . . . . . . . . 74

5.3.2 Adaptation Strategy Comparison . . . . . . . . . . . . . . 76

5.3 .3 Reynolds Number Variations . . . . . . . . . . . . . . . . 78

5.4 Cylinder Test Case . . . . . . . . . . . . . . . . . . . . 87

5.5 Computational Cost . . . . . . . . . . . . . . . . . . . . . 92

6 Conclusions and Outlook $\quad 95$

6.1 Conclusions . . . . . . . . . . . . . . . . . . . . 95

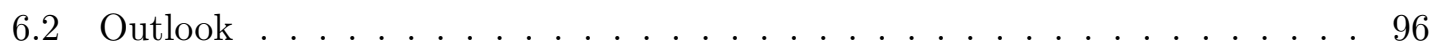

$\begin{array}{lr}\text { A Validation and Scaling } & 97\end{array}$

A.1 Comparison of Compressible and Incompressible Solver _ . . . . . . . 97

A.2 Numerical Experiments . . . . . . . . . . . . . . . . . . . . . . 98 
A.2.1 Laminar Boundary Layer on a Flat Plate . . . . . . . . . . . . . 99

A.2.2 Flow over a NACA0012 and NACA0015 Profile . . . . . . . . . . 100

A.2.2.0.1 NACA0012 . . . . . . . . . . . . 100

Bibliography 



\section{List of Figures}

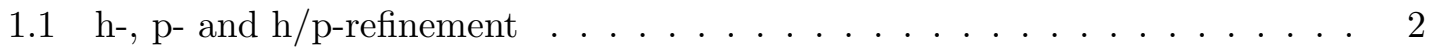

$2.1 \log \left|a_{k}\right|$ vs $k$. for four different examples of convergence. Algebraic convergence: $a_{k} \sim 1 / k^{2}$, Subgeometric convergence: $a_{k} \sim \exp \left(-1.5 k^{2 / 3}\right)$, Geometric convergence: $a_{k} \sim \exp (-\mu k)$ for any positive $\mu$, Supergeometric convergence: $a_{k} \sim \exp (-k \cdot \log (k))$ or faster. . . . . . . . . . . 13

2.2 Periodic extension of three different functions. . . . . . . . . . . . . . . . . 14

2.3 Fourier coefficients vs $k$ for the three different functions. Log-linear (left) and $\log -\log ($ right $) \ldots \ldots \ldots \ldots \ldots \ldots$

2.4 Subdivision of the physical domain into k elements. The DGSEM does not enforce continuity at the element boundaries. A Riemann problem is solved to determine the value of the fluxes at the boundaries. . . . . . . . . . 28

4.1 Error estimations for two cases: optimal scaling (square) and suboptimal scaling (triangle). . . . . . . . . . . . . . . . . . . 45

4.2 Example of the extrapolation process within the adaptation procedure, to determine the polynomial order based on the truncation error: optimal scaling (squares) and suboptimal scaling (triangles). Dashed horizontal line: truncation error threshold and black line: least square extrapolation. . . . . 48

4.3 Truncation error estimation for different combinations of polynomial orders (left) and corresponding LDoF (right) . . . . . . . . . . . . . . 53

4.4 Illustration of truncation error plane fitting used for 2D extrapolation, Polynomial orders $N_{x}$ and $N_{y}$ vs truncation error value $\log (T E)$ (different color

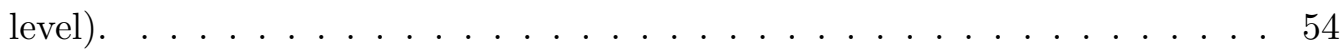

4.5 Truncation error estimation for different combinations of polynomial orders (left) with extrapolated values and corresponding $\mathrm{LDoF}$ (right). . . . . . . 55

4.6 Truncation error with without extrapolation (left) and extrapolated values (right) . . . . . . . . . . . . . . . . . . . 5 55

5.1 The function $\rho(x, y)=p(x, y)=e^{-5\left(4(x-0.5)^{2}+(y-0.5)^{2}\right)}$ is used for the source term of the manufactured solution. . . . . . . . . . . . . . . . 61 
5.2 The truncation error estimation $\tau_{8}^{7}$ (left) and the exact truncation error $\tau_{\text {exact }}^{7}$ (right) on a $4 \times 4$ mesh (top) and 10x10 mesh (bottom) for polynomial order $N_{x}=N_{y}=7$. Colored contours show logarithmic values for the errors.

5.3 Scatter plot (logarithmic scale) for the truncation error $\tau_{8}^{N}$ for varying polynomial orders $N_{x}, N_{y}$ in the $4 \times 4$ grid and the manufactured solution case, only showing element A of Figure 5.2: left shows the estimated error and right shows the exact error.

5.4 Scatter plot (logarithmic scale) for the truncation error $\tau_{8}^{N}$ for varying polynomial orders $N_{x}, N_{y}$ in the 10x10 grid and the manufactured solution case, only showing element B of Figure 5.2: left shows the estimated error and right shows the exact error. . . . . . . . . . . . . . . . . . . . 64

5.5 Logarithm of the estimated and exact truncation errors as a function of the polynomial order $N$ for $N=1, \ldots, 7$. The estimation is performed with a fine polynomial order $P=8$. Results are shown for the highlighted element A of the $4 \times 4$ mesh (left) and element B of the 10x10 mesh, see Figure 5.2. . 65

5.6 Truncation error estimation $\tau_{8}^{N}$ for $N=1, \ldots, 7$ : a posteriori, quasi-a priori without correction and quasi-a priori with correction for the manufactured solution test case $(10 \mathrm{x} 10 \mathrm{mesh}) \ldots \ldots \ldots \ldots \ldots$

5.7 Adaptation results for different required truncation errors (logarithmic scale); The computation Legendre-Gauss nodes are shown in each element. . . . . 66

5.8 NACA0012 simulation, $\rho u . \ldots \ldots \ldots$. . . . . . . . . . 67

5.9 Adapted meshes using the discretisation error ("feature based") strategy with thresholds $10^{-2}$ (top) and $10^{-3}$ (bottom) . . . . . . . . . . . 68

5.10 Adapted meshes based on truncation error, TE (left) and isolated truncation error, ITE (right) for thresholds $10^{-4}$ (top) and $10^{-5}$ (bottom). . . . .

5.11 Adapted meshes based on different refinement criteria with comparable final number of DoF. Top is anisotropic truncation error based $\left(10^{-4}\right.$ threshold, $20480 \mathrm{DoF})$. Middle is anisotropic isolated truncation error based $\left(10^{-5}\right.$ threshold, $21444 \mathrm{DoF})$. Bottom is isotropic discretisation error based $\left(10^{-2}\right.$ threshold, 19096 DoF) . . . . . . . . . . . . . . . . 70

5.12 Lift and drag coefficients for NACA0012 based on different adaptation algorithms. Uniform refinement, Discretisation error (DE), Truncation error (TE) and Isolated Truncation error (ITE) adaptation strategies are shown. 72

5.13 Boundary layer simulation $\rho u$ with singularity at $x=4 \ldots \ldots$. . . . . 73

5.14 Truncation error $\tau_{8}^{7}$ based on the density (logarithmic scale), for boundary layer simulation, singularity at $x=4 \ldots \ldots \ldots \ldots \ldots 74$

5.15 Adapted boundary layer meshes based on various truncation error thresholds: $10^{-1}$ (upper-left), $10^{-2}$ (lower-left) and $10^{-3}$ (upper-right). The computation Legendre-Gauss nodes are shown in each element. 
5.16 Error estimation and required error for elements A (top), B (middle) and C (bottom) of Figure 5.15, linear error extrapolation is used on elements B and C. Horizontal dashed line shows the $10^{-3}$ threshold. . . . . . . . . . . . 79

$5.17 c_{D}$ error based on different simulations on the corresponding DoF; uniform polynomial order solutions (blue with polynomial order in the box) and solutions obtained by the $\tau$-truncation error adaptation process (red with the adaptation criterion $\tau_{\text {max }}$ in the box $)$ P Plotted value $\frac{\left|c_{D}-c_{D(P=10)}\right|}{\left|c_{D(P=10)}\right|}$, where $c_{D(P=10)}$ is calculated on a uniformly refined mesh with $P=10 \ldots \ldots 80$

5.18 Adapted meshes based on discretisation error approach for the adaptation criteria $10^{-1}$ (top), $10^{-2}$ (middle), $10^{-3}$ (bottom). . . . . . . . . . . 8

5.19 Adapted meshes based on the truncation error (left) and the isolated trun-

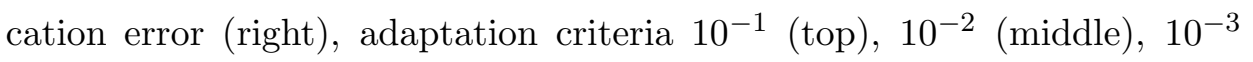

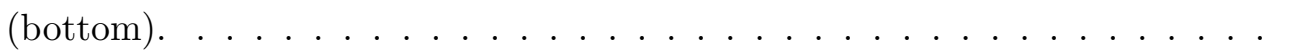

5.20 Drag value $C_{D}$ and DoF based on different adaptation strategies. Uniform refinement, Discretisation error (DE), Truncation error (TE) and Isolated Truncation error (ITE) strategies are shown. . . . . . . . . . . . . 83

5.21 Boundary layer profile and node positions for different truncation error thresholds. . . . . . . . . . . . . . . . . . . . . . 84

5.22 Adaptation based on the truncation error and different Reynolds numbers. . 85

5.23 Adaptation based on the isolated truncation error and different Reynolds numbers. . . . . . . . . . . . . . . . . 86

5.24 Cylinder simulation, $\rho u$ for $P=8 \ldots \ldots \ldots \ldots$. . . . . . . 88

5.25 Adaptation results based on a converged solution with $P=6$ with extrapolation (left) and based on a solution with $P=8$ with no extrapolation, $\tau_{\max }=10^{-1}$ was used as the adaptation criterion. . . . . . . . . 88

5.26 Adaptation based on the truncation error and different Reynolds numbers. . 90

5.27 Adaptation based on the isolated truncation error and different Reynolds numbers. . . . . . . . . . . . . . . . . . . 91

5.28 Relative error in drag coefficient $\frac{\left|c_{D}-c_{D(P=10)}\right|}{\left|c_{D(P=10)}\right|}$ and computational time (in seconds). The reference drag $c_{D(P=10)}$ is calculated on a uniformly refined mesh with $P=10$. The blue line shows uniform polynomial order (non-adapted meshes). Adapted simulations include: a posteriori approach (orange), quasi-a priori approach without correction (green) and quasi- $a$ priori corrected (red). The number of DoF for each simulation is shown in the boxes. . . . . . . . . . . . . . . . . . . . . . . . . 94

A.1 Comparison of compressible and incompressible solvers on a Boundary Layer; streamwise-velocity for $R e_{L_{p}}=600$.

A.2 Velocity profiles at a distance of $L=5.2$ downstream of the leading edge at a Reynolds number of $R e_{L}=520 \ldots \ldots \ldots 1$ 
A.3 Drag error for the flat plate at $R e_{L_{p}}=3600 \ldots \ldots \ldots \ldots \ldots$. . . . . 102

A.4 NACA0015 airfoil meshes using triangles for the incompressible and quadrilaterals for the compressible solver. . . . . . . . . . . . . . . . 102

A.5 h/p refinement for NACA0012. . . . . . . . . . . . . . . . . 104

A.6 Pressure (Cp) and skin friction (Cf) coefficients for a NACA0015 at Re $=100$ using compressible and incompressible solvers. . . . . . . . . . . . . 105 


\section{List of Tables}

4.1 Overview of algorithm properties. . . . . . . . . . . . . . . . . 44

4.2 Computational features of the estimation procedures. . . . . . . . . . . 58

5.1 NACA0012 simulation properties . . . . . . . . . . . . . 67

5.2 NACA0012 simulations and DoF . . . . . . . . . . . . . . 71

5.3 Boundary Layer Simulation overview. . . . . . . . . . . . . . . . 73

5.4 Boundary Layer simulation results. . . . . . . . . . . . . . . . . . 78

5.5 Cylinder Simulation overview. . . . . . . . . . . . . . . . 87

5.6 Runtime and speedup of the a posteriori and quasi- $a$ priori adaptation approaches, non-dimensionalized with respect to a homogeneous polynomial $P=10$ (no adaptation), time convergence until $\|\mathcal{R}(\tilde{u})\|_{L_{\infty}}<10^{-10} \ldots \ldots .93$

5.7 Computational cost of a posteriori and quasi- $a$ priori adaptation algorithms non-dimensionalized with respect to a homogeneous simulation with polynomial $P=10$ (no adaptation) . . . . . . . . . . . . . . . 93

A.1 Summary of test cases detailing Reynolds number, Mach number and Angle of Attack (AOA) . . . . . . . . . . . . . . . . . . . 98

A.2 Viscous drag-coefficients $c_{D_{v}}$ at $\operatorname{Re} R e_{L_{p}}=60$ and $R e_{L_{p}}=600$ for compressible, incompressible and Blasius solution. In addition, results for the displacement thickness $\delta_{1}^{R_{e_{L}}=520}$ are summarized. . . . . . . . . . . . 101

A.3 NACA0015 at $R e_{c}=100$ and $\alpha=0^{\circ}$. Drag-coefficients $c_{D}=c_{D_{p}}+c_{D_{v}}$, including pressure $c_{D_{p}}$ and viscous $c_{D_{v}}$ components, for the compressible and incompressible solvers. . . . . . . . . . . . . . . . . 103 



\section{Introduction}

\section{Contents}

$1.1 \quad$ Content . . . . . . . . . . . . . . . . . . . . . 1

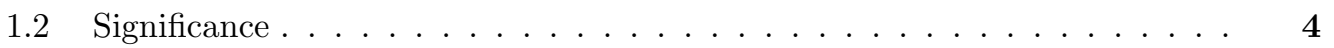

1.3 Publication and Outreach . . . . . . . . . . . . . . . 6

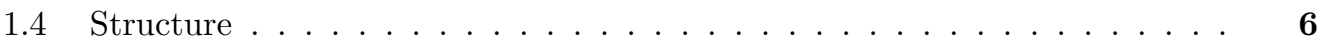

\subsection{Content}

Finite volume, finite difference and finite element numerical methods are regarded as the mainstream and well accepted techniques to solve the Navier-Stokes equations that govern fluid flow (see Peiro and Sherwin [Peiro 2005]). However, during the last decades the interest of the fluid dynamics community has shifted towards high order methods, such as spectral methods or Discontinuous Galerkin, see Wang et al. [Wang 2013] for recent advances. These methods enable the use of high degree polynomials inside each computational element to approximate the numerical solution. By doing so, the accuracy of the solution is improved and the numerical error is shown to decrease exponentially for smooth flows.

Discontinuous Galerkin (DG) methods were developed by the pioneering work of Reed and Hill [Reed 1973] in 1973 in the framework of hyperbolic partial differential equations for the neutron transport equation, and it was only in the late 1990's that the method was generalized to elliptic and convection-diffusion problems; e.g. Bassi and Rebay in 1997 [Bassi 1997], Baumann in 1997 [Baumann 1997] or Cockburn and Shu in 1998 [Cockburn 1998]. Since, DG methods have proven useful in solving the compressible, e.g. [Bassi 1997, Landmann 2008, Nguyen 2007, Oliver 2007, Kopriva 2009], and the incompressible NS equations, e.g. [Bassi 2006, Riviere 2008, Cockburn 2009, Shahbazi 2007, Ferrer 2011, Ferrer 2012b, Ferrer 2014].

The high order DG approach relaxes the continuity requirement needed in continuous methods and allows discontinuities between elements, e.g. non-conforming meshes with hanging nodes or varying polynomial orders. This characteristic enhances the flexibility of the method when dealing with complex flows and eases the incorporation of adaptation strategies. Since discontinuities are allowed in the numerical flow, local adaptation can 
be performed either by locally refining the mesh (h-refinement) or by a local increase in the polynomial order in certain elements (p-refinement), see Figure 1.1. Examples for both strategies may be found in Mavriplis [Mavriplis 1994], Van der Vegt et al. [van der Vegt 2002] or Roy et al. [Roy 2009].

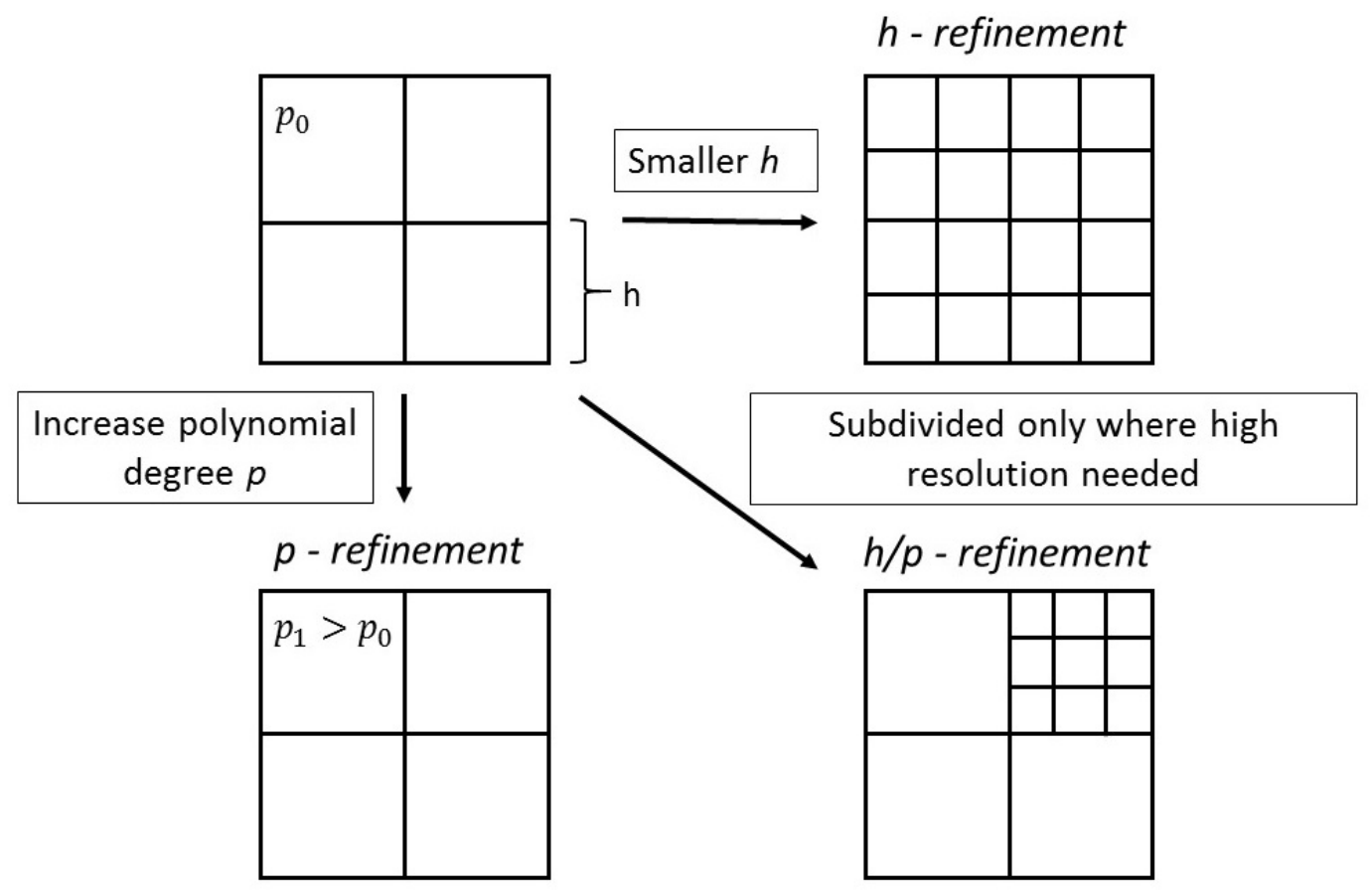

Figure 1.1: h-, p- and h/p-refinement

The flexibility for h- or p-adaptation raises the question of which strategy provides better solutions with a minimal cost. For a broad range of cases with smooth numerical solution, an increase of the polynomial order has shown to outperform h-refinement in terms of accuracy for a given number of degrees of freedom (DoF), e.g. Karniadakis and Sherwin [Karniadakis 1999], Ferrer et al. [Ferrer 2012a].

More important than the local accuracy of the method is to identify the flow regions that require refinement to obtain an optimal global accuracy. An efficient refinement strategy would guarantee high accuracy whilst keeping the computational cost and number of DoF low. The classical and almost naive approach considers creating denser meshes in the flow regions that present geometric complexities or high flow gradients to maximize the accuracy. To identify these zones, density or pressure gradients are often used as the criterion for refinement. This so called "feature based adaptation" has been broadly applied to improve the accuracy around shock waves, expansion fans, contact discontinuities and boundary layers, e.g. Dwight et al. [Dwight 2008], Ainsworth et al. [Ainsworth 2007]. However, the main limitation of this approach resides in the fact that there is no clear and direct relation between the computed feature (used as adaptation criterion) and the numerical errors and therefore the overall accuracy is generally difficult to predict and control. 
To overcome these drawbacks, adjoint based adaptation procedures were developed and have gained popularity during recent years, e.g. Hartmann [Hartmann 2006], Balasubramanian and Newman [Balasubramanian 2009]. These techniques require the selection of a functional target (e.g. lift or drag in airfoil computations) to provide information about the regions that require refinement.

An alternative to adjoint based methods is provided by the truncation error (see Fraysse et al. [Fraysse 2012b] or Derlaga et al. [Derlaga 2015] for comparisons to adjoints). This technique, which is the main topic of this work, is seen as a promising methodology to avoid the high computational cost associated to adjoint methods whilst keeping the favorable fast computation properties and accuracy inherited from error estimation methods. In addition, it should be noted that truncation error based methods do not require the selection of a particular functional and consequently targets the numerical accuracy of all functionals.

The truncation error is defined as the difference between the discrete partial differential equation (PDE) and the exact PDE operator, both applied to the exact solution of the problem, as detailed by Phillips and Roy [Phillips 2011]. Mathematically, this can be written as

$$
\tau^{N}=\mathcal{R}^{N}(u)-\mathcal{R}(u)
$$

where $\tau^{N}$ denotes the truncation error for a polynomial order $N, \mathcal{R}$ is the partial differential operator, $\mathcal{R}^{N}$ the discrete partial differential operator (of order $N$ ) and $u$ represents the exact solution.

The discretization error (i.e. the difference between the exact solution to the PDE and the exact solution to the discretized PDE) and the truncation error are related through the Discretization Error Transport Equation (DETE), see Roy [Roy 2010]. Roy has shown that the truncation error acts as a local source for the discretization error, which is convected and diffused through the domain with the flow. Hence, the resulting discretization error is a combination of the locally generated error and the error transported by flow advection and diffusion. This relationship provides a valid argument for the use of the truncation error as a sensor for a mesh adaptation algorithm, e.g. Syrakos et al. [Syrakos 2012], Frayssee et al. [Fraysse 2012a, Fraysse 2014]. In addition, in high order discretizations, an accurate estimate for the error also enables modification of the polynomial order via a procedure known as $\tau$-extrapolation to better capture the numerical solution (see Bernert [Bernert 1997]).

The truncation error can be estimated using evaluations of the discrete PDE operator on a hierarchy of meshes. Shih and Williams [Shih 2009] proposed a multiple grid method with interpolation from the coarse to the fine grid. More recently, Gao and Wang [Gao 2011] proposed a similar approach with interpolation from low to high polynomial order. The main drawback of the coarse to fine grid approaches is that they tend to over-predict the truncation error, Phillips [Phillips 2014]. The truncation error may be also estimated by means of the $\tau$-estimation method of Brandt [Brandt 2011]. This es- 
timate relies on the evaluation of the fine grid solution (e.g. higher order solution) on a coarse mesh (e.g. space spanned by lower order functions). The fine to coarse approach is more accurate but more costly than the coarse to fine one. The seminal works of Berger [Berger 1987], Bernert [Bernert 1997] and Fulton [Fulton 2003] posed the fundamentals of the $\tau$-estimation method and studied the conditions on the restriction operators to transfer solutions from fine to coarse and vice-versa, mainly for finite difference uniform meshes. Syrakos and Goulas [Syrakos 2006] successfully implemented the $\tau$-estimation method for a finite volume discretization and the incompressible Navier-Stokes equations. Fraysse et al. [Fraysse 2012a, Fraysse 2013] extended these analyses to finite volume discretizations on any kind of meshes, with an interesting extension to non-converged temporal solutions. More recently, Rubio et al. [Rubio 2013] extended this methodology to continuous high-order methods using a spectral collocation method. It was shown that some of the fundamental assumptions about the error tendency, that are well established for low-order methods, are no longer valid when dealing with high-order schemes.

The extensions of $\tau$-estimation to high order discontinuous Galerkin methods have recently been recently performed by Rubio et al. [Rubio 2014] for a simple advection equation. Additionally in [Rubio 2014], a quasi-a priori idea introduced by Fraysse et al. [Fraysse 2013] was adapted to high order discontinuous discretizations using scalar partial differential equations. This quasi- $a$ priori approach enables the computation of the truncation error using solutions that are not fully converged in time. Note that the solution is considered converged (or steady) when the iterative errors are below a certain threshold, normally close to machine roundoff. Obtaining the truncation error before the solution is totally converged saves computational resources while providing valuable information how to perform adaptation.

The objective of this work is to extend the achievements of Rubio et al. [Rubio 2014] for simple differential equations to the compressible Navier-Stokes equations in multiple dimensions. As a result, an efficient and robust algorithms for local p-adaptation using discontinuous Galerkin discretizations is proposed. Converged and non-converged solutions are considered in this analysis and named a posteriori, quasi- $a$ priori and quasi- $a$ priori corrected adaptation strategies, respectively. The accuracy, efficiency and computational costs of the different strategies are included. Finally, the high order technique used in this work enables the anisotropic treatment of the error in terms of the flow direction, hence allowing the p-adaptation refinement to be performed differently depending on the flow orientation.

\section{$1.2 \quad$ Significance}

This work was conducted within the Advances in Numerical and Analytical tools for DEtached flow prediction (ANADE) project (Initial Training Network, PITN-GA-289428) funded under the European Commission' Seventh Framework Programme (FP7) within 
the People work programme (Marie Curie actions). This project aims to contribute to a better understanding of separated flows, the underlying physics and the advancement of new numerical methods, that are more suitable for this kind of problems, see www.anadeitn.eu. ANADE comprises the combination of the predominantly theoretical/numerical capabilities developed by academics with state-of-the-art computational proficiencies available at present, to improve understanding of separated flow.

The overall objectives and significance of this work follows those of ANADE, which are to identify quantified aerodynamic benefits in terms of drag reduction, lifting surface, size reduction (weight and fuel consumption reduction), aircraft operational maneuvers (stall conditions), noise emissions (Green Air Transport Operations) and aircraft operational, safety (tail buffet alleviation), which are of particular importance in the future.

One advanced new numerical method, considered to have high potential to achieve the desired goals of ANADE, is the discontinuous Galerkin approach, based on spectral element methods. This approach containing higher order discretizations was applied in this work, because it is considered to be more suitable to solve complex flows with a high disparity of scales as present in separated flows than the second order schemes presently used. Significantly fewer degrees of freedom are needed to reach the same level of accuracy. On a given mesh, the applied method allows an improved prediction of crucial flow phenomena, such as boundary layers including laminar to turbulent transition, drag forces, wakes, vortical flows and interaction phenomena like the one between wing vortex and tail.

However, because of their computational complexity, these methods have not been competitive up to now and a substantial effort is required to improve their numerical performance.

In particular, this work deals with adaptation strategies for high order discontinuous Galerkin methods. In the past different adaptation methods were developed for high order methods achieving reasonable results with high accuracy. Nevertheless, the disadvantage of those methods are their very expensive cost in terms of computational cost and degrees of freedom in order to describe the flow with the needed accuracy. This is a drawback for large scale simulations as required in industrial applications. Hence, there is a continuous demand and interest for cheap adaptation procedures.

This work contributes to close this gap and presents newly developed techniques for adaptation approaches that are computationally cheaper.

The design of such methods is based on previous work where the truncation error has already been successfully used for low order methods (finite volume methods) as a criterion for mesh adaptation [Fraysse 2013]. Actually, these methods have recently been extended and validated for high order methods by [Rubio 2014], but no adaptation scheme for mesh adaptation has been designed relying on this theory yet. This works makes use of the previously developed theory to design a novel adaptation algorithm for high order methods based on the truncation error.

The newly developed algorithm has been applied to different test cases and, in conse- 
quence, was continuously revised and improved. Moreover, the algorithm was extended by using an error-extrapolation strategy which significantly reduces the computational cost. As an example, for a boundary layer simulation a speedup of a factor of 7.6 could be achieved in comparison to the conventional solution as well.

Further reduction of computational cost could be achieved by modifying the algorithm to be capable of non-converged solutions and minimizing of the DoF.

This led to a significant reduction of DoF for all test-cases. As an example, an $\sim 80 \%$ reduction of DoF can obtained for a NACA0012 airfoil simulation.

\subsection{Publication and Outreach}

During this $\mathrm{PhD}$ thesis various publications have been made in international journals and different presentations at international conferences have been given.

Publications:

- M Kompenhans, E Ferrer, M Chavez, E Valero, "Numerical study of three dimensional acoustic resonances in open cavities at high Reynolds numbers", Aerospace Science and Technology, Vol 45, p 501511, 2015

- M Kompenhans, G Rubio, E Ferrer, E Valero, Adaptation strategies for high order discontinuous Galerkin methods based on Tau-estimation", Journal of Computational Physics, Vol 306, p 216-236, 2016

- M Kompenhans, G Rubio, E Ferrer, E Valero, "Comparisons of p-adaptation strategies for high order discontinuous Galerkin methods", Computer and Fluids, Under review

Presentations:

- M Kompenhans, E Ferrer, "Comparisons of Compressible and Incompressible Solvers: Flat Plate Boundary Layer and NACA airfoils", 2nd international workshop on high order CFD methods, organised by DLR, May 2013, Cologne

- M Kompenhans, G Rubio, E Ferrer, E Valero, "Automatic HP adaptation for discontinuous Galerkin by means of Tau-estimation ", ECCOMAS Barcelona, 20 -25 July 2014, Barcelona

- M Kompenhans, G Rubio, E Ferrer, E Valero, "Adaptation Strategies for discontinuous Galerkin by Means of Tau-Estimation", 13th US National Congress on Computational Mechanics San Diego, July 27 - 30, 2015

\subsection{Structure}

This work is organized as follows 
Chapter 2 In chapter 2 the basic theory for Spectral Element Methods is introduced. General guidelines regarding the convergence are given for a discrete Fourier expansion and for Spectral Element Methods. Finally, the discontinuous Galerkin method is detailed. Additionally, some information about the scaling of computational cost and computational errors are given. Furthermore, a validation and comparison with other solvers are shown.

Chapter 3 Chapter 3 deals with different errors that occur in numerical methods. More precisely, discretisation error, iteration error and truncation error will be explained. These errors are of importance to develop the adaptation method presented in the following chapters.

Chapter 4 In chapter 4 the complete adaptation process is detailed. In the first part the isotropic algorithm is explained. Furthermore, this approach is extended by an extrapolation procedure. In the second part the algorithm is advanced to be capable of anisotropic refinement. The quasi a-priori estimation scheme is incorporated and strategies to minimize the DoF are shown.

Chapter 5 In chapter 5 results for the proposed adaptation algorithms are shown. First the algorithms are verified using a manufactured solution test case with the Euler equations. Then an inviscid NACA test case is used to present adaptation results and scaling. Furthermore, the adaptation process is applied on compressible Navier Stokes equations using the flat plate boundary layer test case. Finally, efficiency of the methods is quantified. The run-time of the different approaches is compared and the speedup of the proposed adaptation determined.

Chapter 6 Conclusions and an outlook are given in chapter 6 . 



\section{Spectral Methods}

\section{Contents}

2.1 The Fourier Expansion . . . . . . . . . . . . . . . . . . . . . . 10

2.1 .1 The Continuous Fourier Expansion . . . . . . . . . . . . . 10

2.1.2 Discrete Truncated Series . . . . . . . . . . . . . . . . 11

2.1 .3 Orders of Convergence . . . . . . . . . . . . . . . . . 11

2.1.4 Fourier Series Convergence _. . . . . . . . . . . . . . . . 13

2.1.5 Fourier Quadrature . . . . . . . . . . . . . . . . . . . 15

2.1.6 Fourier Interpolation . . . . . . . . . . . . . . . . . 16

2.1 .7 Fourier Interpolation Error $\ldots \ldots \ldots \ldots$

2.1.8 Differentiation through Fourier Interpolation $\ldots \ldots \ldots \ldots$

2.2 Polynomial Basis Functions . . . . . . . . . . . . . . . . . . . 18

2.2 .1 Polynomial Series . . . . . . . . . . . . . . . . . . . 20

2.2 .2 Polynomial Quadrature . . . . . . . . . . . . . . . . . 20

2.2 .3 Orthogonal Polynomial Interpolation . . . . . . . . . . . . . . 22

2.3 Approximation of PDE Solutions _ . . . . . . . . . . . . . . . 25

2.4 Discontinuous Galerkin Spectral Element Method . . . . . . . . . . . . 27

2.5 Implementation and Verification . . . . . . . . . . . . . . . 30

This chapter contains a review about of the basic spectral element theory. Most of the examples and the presented theory can be found in established references for spectral methods [Boyd 1989, Canuto 2006, Kopriva 2009]. Furthermore, this chapter is based on the theory presented in [Rubio 2015], which have been the starting point of the work for this thesis.

The final goal of this thesis is to develop an accurate and efficient adaptation algorithm based on truncation errors for spectral element methods. Therefore, the errors that are relevant in this process have to be introduced. Hereby the focus lies on the convergence of those errors in spectral methods.

The underlying idea of spectral methods is to approximate any function (i.e. the solution of a PDE) as a sum of terms composed of orthogonal functions multiplied by a coefficient. So called basis functions are chosen to ensure an accurate approximation of the corresponding function with a minimum number of summands. 
The most established spectral basis is the Fourier basis, which is only applicable to approximate periodic functions. Later on other bases are introduced, which can deal with non periodic problems in finite domains as well.

This chapter is organized as follows. The first part, section 2.1.1, explains how to approximate functions with a continuous Fourier approach. The classifications of different convergence rates, which will be of importance in the following chapters, are explained. In the second part 2.1.1, the properties of the Fourier continuous expansion are explained for the discrete form. Afterwards, in section 2.2, it will be explained how to use general and non-periodic basis functions. The complete Discontinuous Galerkin Spectral Element Method (DGSEM), which will be used throughout this work, is derived in section 2.4. Finally, some information about the scaling and verification of this method is given in section 2.5 .

\subsection{The Fourier Expansion}

\subsubsection{The Continuous Fourier Expansion}

It is possible to represent any square integrable complex-valued function, $f \in L^{2}(0,2 \pi)$, by its Fourier series

$$
f(x)=\sum_{k=-\infty}^{\infty} c_{k} e^{i k x}
$$

where $c_{k}$ are the complex Fourier coefficients defined by

$$
c_{k}=\frac{1}{2 \pi} \int_{0}^{2 \pi} f(x) e^{-i k x} d x
$$

Equations (2.1-2.2) are a consequence of the orthogonality property of the set of functions $\phi_{k}(x)=e^{i k x}$ in the $L^{2}(0,2 \pi)$ space. $L^{2}(0,2 \pi)$ describes a complex Hilbert space on which the inner product

$$
(u, v)=\int_{0}^{2 \pi} u(x) \overline{v(x)} d x
$$

and norm

$$
\|u\|=\left(\int_{0}^{2 \pi}|u(x)|^{2} d x\right)^{1 / 2} .
$$

are defined. Accordingly, the orthogonality relation between $\phi_{j}(x)=e^{i j x}$ and $\phi_{k}(x)=e^{i k x}$ is described by

$$
\left(\phi_{j}(x), \phi_{k}(x)\right)=\int_{0}^{2 \pi} e^{i j x} e^{-i k x} d x=2 \pi \delta_{j k} .
$$

Equation (2.3) denotes that each of the terms of the Fourier series, i.e. $c_{k} e^{i k x}$, is the projection of the function onto the corresponding Fourier polynomial.

Based on Euler's formula $e^{i x}=\cos (x)+i \sin (x)$, a Fourier cosine transform and a 
Fourier sine transform can be introduced

$$
\begin{aligned}
& a_{n}=\frac{1}{2 \pi} \int_{0}^{2 \pi} f(x) \cos (n x) d x, \\
& b_{n}=\frac{1}{2 \pi} \int_{0}^{2 \pi} f(x) \sin (n x) d x .
\end{aligned}
$$

Because of the Fourier expansion, a periodic extension of the function is assumed outside of the interval $[0,2 \pi]$. This originates from the periodicity of the basis functions and the chosen scalar product. Consequently the result of the projection of the function $f(x)$ using (2.2) results not in the projection of the function, but the projection of the periodic extension of the function. This fact influences the rate of convergence of the Fourier series.

\subsubsection{Discrete Truncated Series}

Due to the infinite character of the Fourier series (2.1) it is necessary to truncate the series for practical problems,

$$
f(x)=\sum_{k=-\infty}^{\infty} c_{k} e^{i k x}=\sum_{k=-N / 2}^{N / 2} c_{k} e^{i k x}+\sum_{|k|=N / 2+1}^{\infty} c_{k} e^{i k x}=P_{N} f(x)+\tau,
$$

where $\tau$ is the truncation error resulting from the truncation of the high order terms of the series. Considering the orthogonality of the basis functions and with $\| e^{i k x}||^{2}=2 \pi$, it can be shown that

$$
\|\tau\|^{2}=\left\|f(x)-P_{N} f(x)\right\|^{2}=2 \pi \sum_{|k|=N / 2+1}^{\infty}\left|c_{k}\right|^{2}
$$

holds for the truncation error of the Fourier series.

Not only the convergence of Fourier series is of high importance, furthermore one would like to know how rapidly the coefficients decay to zero $\left(c_{k} \rightarrow 0\right)$. As a general rule it holds that the smoother the function, the more rapidly its spectral coefficients converge [Boyd 1989]. Since Fourier approximations imply periodic extension of an approximated function $f(x)$, only periodic functions will produce smooth periodic extensions. That means that the Fourier series shows exponential convergence primarily for analytic and periodic functions. The different rates of convergence of the Fourier series are explained in the following section.

\subsubsection{Orders of Convergence}

In the following the different orders of convergence are explained. 


\section{Algebraic Convergence}

The algebraic index of convergence $\mathrm{m}$ is the largest number for which

$$
\lim _{k \rightarrow \infty}\left|a_{k}\right| k^{m}<<\infty \quad, k>>1 .
$$

where the $a_{k}$ are the coefficients of the series (in case of a Fourier series the limitations holds for $a_{n}$ and $b_{n}$ Alternative definition: if the coefficients of a series are $a_{k}$ and if the relation

$$
a_{k} \backsim O\left(1 / k^{m}\right) \quad, k>>1
$$

holds, then $m$ is the algebraic index of convergence.

\section{Exponential Convergence}

If the algebraic index of convergence $m$ is unbounded (which means that the coefficients $a_{k}$ decrease faster than $1 / k^{m}$ for any finite power of $\mathrm{m}$ ) then the series has the property of infinite order, exponential or spectral convergence. Alternative definition: the series has infinite order or exponential convergence when

$$
a_{k} \backsim O\left(\exp \left(-q k^{r}\right)\right) \quad, k>>1
$$

with $q$ a constant for some $r>0$.

\section{Exponential Index of Convergence}

The exponential index of convergence $r$ is given by an alternative definition:

$$
r=\lim _{k \rightarrow \infty} \frac{\log \left|\log \left(\left|a_{k}\right|\right)\right|}{\log (k)}
$$

An equivalent definition is that if $s$ and $q>0$ are constants and

$$
a_{k} \backsim O\left(s \exp \left(-q k^{r}\right)\right) \quad, k>>1
$$

then the exponential index of convergence is the exponent $r$.

\section{Rates of exponential convergence}

A series whose coefficients are $a_{k}$ is said to have the property of supergeometric, geometric or subgeometric convergence depending upon whether

$$
\lim _{k \rightarrow \infty} \log \left(\left|a_{k}\right|\right) / k= \begin{cases}\infty & \text { supergeometric } \\ \text { const. } & \text { geometric } \\ 0 & \text { subgeometric }\end{cases}
$$



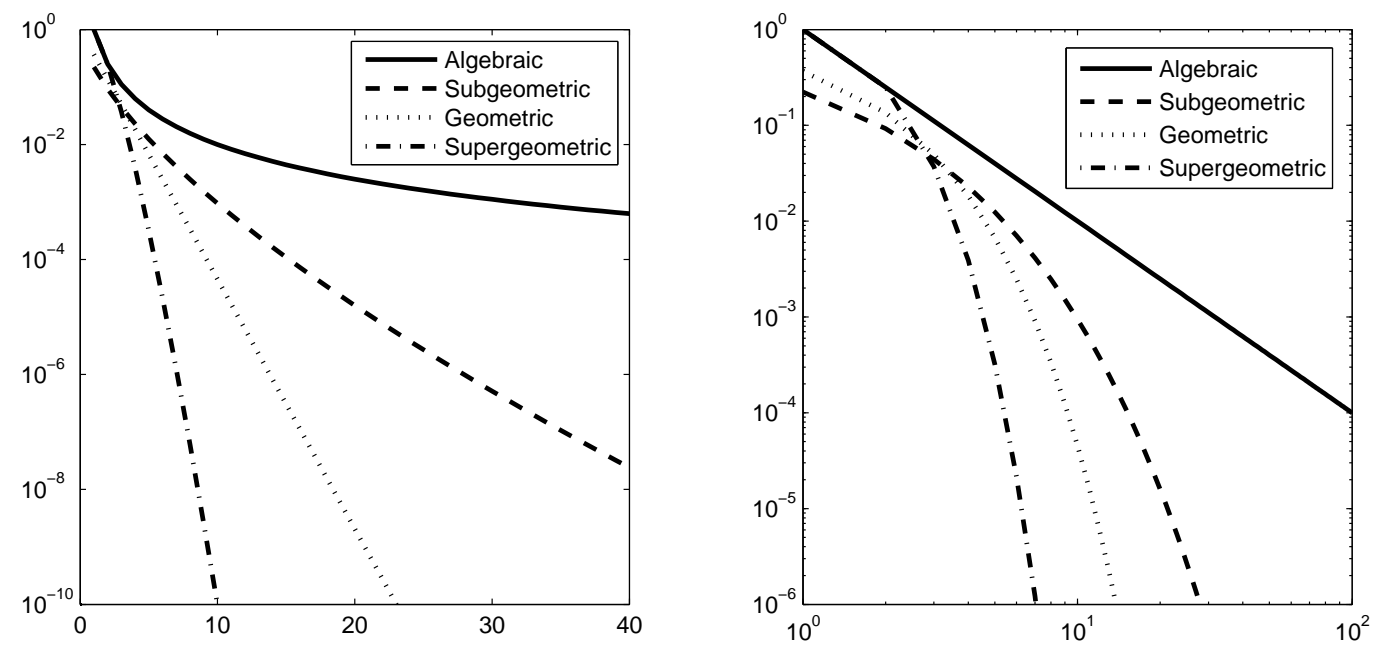

Figure 2.1: $\log \left|a_{k}\right|$ vs $k$. for four different examples of convergence. Algebraic convergence: $a_{k} \sim 1 / k^{2}$, Subgeometric convergence: $a_{k} \sim \exp \left(-1.5 k^{2 / 3}\right)$, Geometric convergence: $a_{k} \sim \exp (-\mu k)$ for any positive $\mu$, Supergeometric convergence: $a_{k} \sim \exp (-k \cdot \log (k))$ or faster.

\section{Asymptotic rate of geometric convergence}

According to [Boyd 1989], if a series has geometric convergence, that means an expansion has the exponential index of convergence $r=1$, so that

$$
a_{k} \backsim \alpha \exp (-k \rho)
$$

where $a_{k}$ are the spectral coefficients, $\rho$ a constant and $\alpha$ an unspecified factor, that varies more slowly with $k$ than the exponential (such as $k^{m}$ for some $m$ ), then the asymptotic rate of geometric convergence is $\rho$. Equivalently,

$$
\rho=\lim _{k \rightarrow \infty}\left(-\log \left|a_{k}\right| / k\right) .
$$

This definition is meaningful only for geometrically converging series; it does not apply if the algebraic index of convergence is smaller than infinity nor if the exponential index of convergence is smaller than $r$.

\subsubsection{Fourier Series Convergence}

To illustrate a given convergence rate of the Fourier Series and the influence of the smoothness of a function three functions are shown as a demonstration. The coefficients of the 


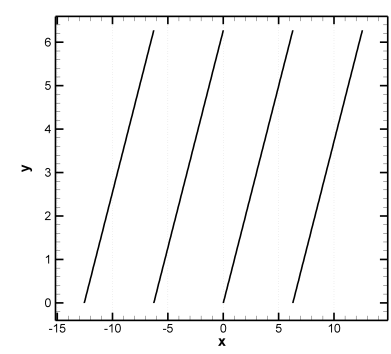

(a) $f_{1}=x$

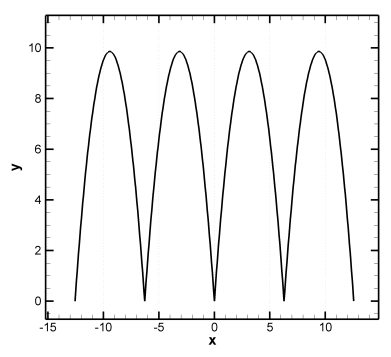

(b) $f_{2}=x(2 \pi-x)$

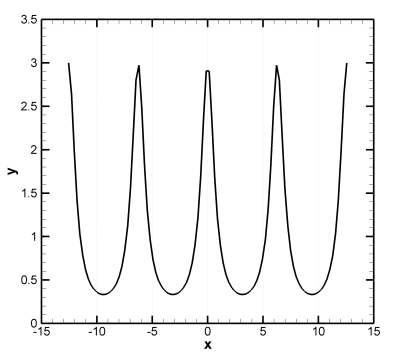

(c) $f_{3}=3 /(5-4 \cos (x))$

Figure 2.2: Periodic extension of three different functions.

Fourier series are calculated for the following functions.

$$
\begin{aligned}
& f_{1}=x \\
& f_{2}=x(2 \pi-x), \\
& f_{3}=\frac{3}{5-4 \cos (x)} .
\end{aligned}
$$

The same example can be found in [Canuto 2006] [Kopriva 2009] or [Rubio 2015]. Fig. 2.2 shows the periodic expansions of the three functions. The functions present different degrees of smoothness. The first function, $f_{1}$, is continuous within the interval $(0,2 \pi)$, however it is non periodic and its periodic expansion is not continuous in the whole domain. In contrast, the periodic expansion of the second one, $f_{2}$, is continuous in $\mathcal{C}^{0}$ (where $\mathcal{C}^{r}$ denotes the set of continuous functions that have $r$ continuous derivatives). Finally, the periodic expansion of the function $f_{3}$ is $\mathcal{C}^{\infty}$.

Fig. 2.3 shows the decay rate of the coefficients of the Fourier expansions for the three functions (absolute value in log-linear (left) and log-log (right)). As it can be seen, $f_{1}$ and $f_{2}$ result in an algebraic convergence displayed by the straight line in the log-log plot, with slope $m=1$ and $m=2$. On the other hand, $f_{3}$ converges exponentially represented by the straight line in the log-linear plot.

The orders of convergence are only relevant and representative asymptotically (for a large number of basis functions, $k$ ). For low $k$ the slope of the decay might be not be significantly better (see $f_{3}$ in comparison to $f_{2}$ or $f_{1}$ in Figure 2.3. Furthermore, the spectral convergence means how rapidly the coefficients decrease for $k \rightarrow \infty$ for a fixed approximated function $f(x) \in \mathcal{C}^{m}$, and not how rapidly the coefficients decrease for $m \rightarrow \infty$ with fixed $k$. If the coefficients are computed numerically, the asymptotic order may be defeated by the Roundoff Plateau.

The importance of this result is highlighted in the following sections, where it is shown that the convergence of spectral methods is linked to the convergence of the coefficients of the series. 

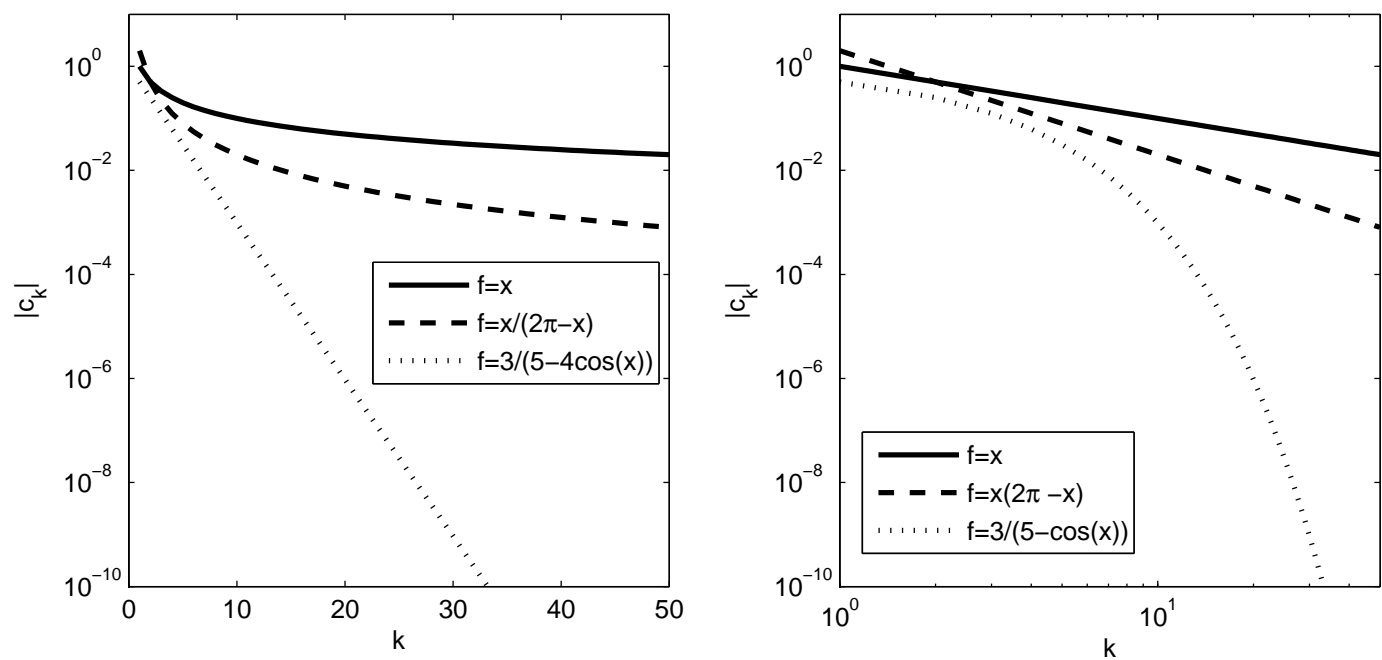

Figure 2.3: Fourier coefficients vs $k$ for the three different functions. Log-linear (left) and $\log -\log$ (right).

\subsubsection{Fourier Quadrature}

In most practical cases it is impossible to apply the continuous Fourier approach (2.2), which can involve the solution of complicated or not solvable integrals. Anyhow, is still necessary to numerically calculate the series coefficients. To evaluate the integrals a quadrature rule can be used. Nevertheless, the quadrature rule has to be appropriate to reproduce the inherent properties of spectral methods. Particularly this means to maintain the spectral accuracy and orthogonality in order to obtain a fast convergence and a coefficient expansion by orthogonal projection.

A quadrature rule approximates an integral of a function based on a sum expression. The sum consists of function values at certain points multiplied by the corresponding weights,

$$
\int_{a}^{b} u(x) d x=Q[u]+E=\sum_{j=0}^{N} u\left(x_{j}\right) w_{j}+E .
$$

To minimize the error $E$ in $(2.18)$ the nodes $x_{j}$ and the weights $w_{j}$ can be optimized.

A quadrature rule under which as many Fourier basis functions as possible remain orthogonal, is the composite trapezoidal rule which has $N$ equispaced nodes. The composite trapezoidal rule integrates exactly the complex exponentials $e^{i k x}$ for $k=0, \pm 1, \pm 2 \ldots, \pm(N-$ 1). On the other hand, the exact result of the integral cannot be assured for $|k|>N-1$, since the product of $e^{i m x}$ and $e^{i n x}$ is nonzero for $n-m= \pm p N$. The quadrature error for higher $k^{\prime} s$ results in an aliasing error (described later). 
The Fourier quadrature rule in the interval $[0,2 \pi]$ is

$$
Q_{F}[u]=\frac{2 \pi}{N} \sum_{j=0}^{N-1} u\left(x_{j}\right), \quad x_{j}=2 j \pi / N .
$$

which is the composite trapezoidal rule with equispaced nodes for periodic functions on the interval $[0,2 \pi]$. A more detailed explanation can be found in [Kopriva 2009].

\subsubsection{Fourier Interpolation}

Based on the Fourier quadrature rule (2.19) the Fourier interpolant can be introduced as

$$
I_{N} f(x)=\sum_{k=-N / 2}^{N / 2-1} \tilde{c}_{k} e^{i k x}
$$

The coefficients of the interpolant can be computed using (2.2)

$$
\tilde{c}_{k}=\frac{2 \pi}{N} \sum_{j=0}^{N-1} f\left(x_{j}\right) e^{-i k x_{j}}, \quad x_{j}=2 j \pi / N .
$$

It can be shown that (2.20) fulfills

$$
I_{N} f\left(x_{j}\right)=f\left(x_{j}\right), \quad j=0,1, \ldots, N-1,
$$

which is the trigonometric interpolant of $f(x)$, see [Kopriva 2009] for more details. It is useful to rewrite $(2.20)$ as

$$
I_{N} f(x)=\sum_{j=0}^{N-1} f\left(x_{j}\right) h_{j}(x)
$$

where $h_{j}$ are the Cardinal functions $\left(h_{j}\left(x_{n}\right)=\delta_{j, n}\right.$ for $\left.|n-j|<N\right)$

$$
h_{j}(x)=\frac{1}{N} \sum_{k=-N / 2}^{N / 2-1} e^{i k\left(x-x_{j}\right)}, \quad j=0,1, \ldots, N-1 .
$$

It should be noted that (2.23) is equivalent to $(2.20)$, where the unknowns are the function values in a set of nodes instead of the coefficients of the modes. The former approach is known as modal in the literature and the latter as nodal.

\subsubsection{Fourier Interpolation Error}

Most certainly the quadrature rule of the previous section introduces an additional source of error due to the fact that it is only an approximation. Consequently, the Fourier interpolation, (2.23), results in a less accurate approximation than the truncated Fourier series with the exact coefficients, (2.2). Comparing the accuracy of the exact Fourier series 
of a function $f(x)$

$$
f(x)=\sum_{k=-\infty}^{\infty} c_{k} e^{i k x}
$$

to the Fourier interpolant of order $N / 2$

$$
I_{N} f(x)=\sum_{k=-N / 2}^{N / 2-1} \tilde{c}_{k} e^{i k x}
$$

leads to

$$
f(x)-I_{N} f(x)=\sum_{|k|=N / 2}^{\infty} c_{k} e^{i k x}+\sum_{k=-N / 2}^{N / 2-1} \sum_{\substack{j=-\infty \\ j \neq 0}}^{\infty} c_{k+j N} e^{i k x}=\tau+R_{N} f .
$$

where the Fourier interpolant coefficients and the Fourier series coefficients have been obtained by substituting the definition of the Fourier series, (2.1), into the definition of the Fourier interpolant coefficients (2.21), see [Rubio 2015] for a detailed derivation. In $2.27 \tau$ is the truncation error defined in (2.8) and $R_{N} f$ is known as aliasing error. It should be noted that the truncation error $\tau$ and the aliasing error $R_{N} f$ are orthogonal, due to the orthogonality of the Fourier series, accordingly

$$
\left\|f-I_{N} f\right\|^{2}=\left\|f-P_{N} f\right\|^{2}+\left\|R_{N} f\right\|^{2} .
$$

Accordingly the approximation obtained using the Fourier interpolation will never achieve more accurate results than the one obtained by using the Fourier truncated series (see [Canuto 2006] for more details). Furthermore, considering (2.27) and that the Fourier basis functions $e^{i k x}$ are bounded by one,

$$
\left|f(x)-I_{N} f(x)\right| \leq 2\left\{\sum_{|k|=N / 2}^{\infty}\left|c_{k}\right|\right\} .
$$

which states that an upper bound for the interpolation error is twice the amount of the truncation error. Furthermore, for spectral accuracy and in the asymptotic range and for sufficiently smooth functions the coefficients behave like $\left|c_{n+1}\right|<<\left|c_{n}\right|$ and thus the error made in the Fourier interpolation is bounded.

$$
\left|f(x)-I_{N} f(x)\right| \sim c_{N+1} .
$$

Certainly, this result is only valid for smooth functions and within the asymptotic range of convergence. 


\subsubsection{Differentiation through Fourier Interpolation}

The approximaton of a derivative of a function can be obtained by differentiating its interpolant (2.23). For the Fourier expansion this results in a multiplication by $(i k)^{l}$ where $l$ is the order of differentiation,

$$
\left(I_{N} f\right)^{(l)}=\sum_{k=-N / 2}^{N / 2-1}(i k)^{l} \tilde{c}_{k} e^{i k x}
$$

Comparing the differentiation error made in the approximation of the derivative with the error made in the approximation of the function leads to

$$
f^{(l)}(x)-\left(I_{N} f(x)\right)^{(l)}=\sum_{|k|=N / 2}^{\infty}(i k)^{l} c_{k} e^{i k x}+\sum_{k=-N / 2}^{N / 2-1} \sum_{\substack{j=-\infty \\ j \neq 0}}^{\infty}(i k)^{l} c_{k+j N} e^{i k x}
$$

Considering the orthogonality of the Fourier basis, the following statement holds

$$
\left|f^{(l)}(x)-\left(I_{N} f(x)\right)^{(l)}\right| \leq 2\left\{\sum_{|k|=N / 2}^{\infty} k^{l}\left|c_{k}\right|\right\} .
$$

Comparing (2.29) with (2.33) shows that an increase of $l$, the order of differentiation, downgrades the approximation by an algebraic factor of $k^{l}$, see [Rubio 2015] and [Canuto 2006] for more details.

\subsection{Polynomial Basis Functions}

Nevertheless the Fourier series provides exponential convergence for periodic functions. These feature cant be guranteed for non-periodic functions. Alternative approaches exist to prevent the exponential convergence characteristic for non-periodic functions.

One approach are Jacobi polynomials, $P_{k}^{(\alpha, \beta)}(x)$, which are a group of polynomials of the form

$$
P_{k}^{(\alpha, \beta)}(x)=\frac{1}{2^{k}} \sum_{i=0}^{k}\left(\frac{k+\alpha}{i}\right)\left(\frac{k+\beta}{k-i}\right)(x-1)^{i}(x+1)^{k-i} .
$$

These functions are orthogonal with respect to the weight $w(x)=(1-x)^{\alpha}(1+x)^{\beta}$ on the interval $(-1,1)$,

$$
\int_{-1}^{1} P_{k}^{(\alpha, \beta)}(x) P_{m}^{(\alpha, \beta)}(x) w(x) d x=\delta_{k m}
$$

Jacobi polynomials can be found as a solution of the Sturm-Liouville problem or applying the Gram-Schmidt orthogonalization method with respect to the weight. This group of polynomials is complete in the space $L_{w}^{2}(-1,1)$ and forms an orthogonal basis with the 
inner product

$$
(u, v)_{w}=\int_{-1}^{1} u(x) v(x) w(x) d x
$$

and norm

$$
\|u\|_{w}=\left(\int_{-1}^{1}|u(x)|^{2} w(x) d x\right)^{1 / 2} .
$$

Furthermore, the decay rate of the coefficients of the truncated series depends only on the smoothness of the function. No requirement for periodicity at the boundaries is needed [Canuto 2006]. Consequently, they are a good alternative to Fourier based approaches for non periodic functions. The most important ones are Legendre polynomials with $\alpha=0$ and $\beta=0, L_{k}(x)=P_{k}^{(0,0)}(x)$ and Chebyshev polynomials, where $\alpha=-1 / 2$ and $\beta=-1 / 2, T_{k}(x)=P_{k}^{(-1 / 2,-1 / 2)}(x)$. For Legendre polynomials the associated weight function is $w(x)=1$, which results in an easy evaluation of integrals such as (2.36). Chebyshev polynomials have powerful approximation properties and the theory for Fourier polynomials also holds for these kind of polynomials (with small changes).

\section{Chebyshev Polynomials}

As stated above, Chebyshev polynomials are a special case of Jacobi polynomials for $\alpha=\beta=-1 / 2$ and satisfy the three-term recursion rule,

$$
T_{k+1}(x)=2 x T_{k}(x)-T_{k-1}(x),
$$

with $T_{0}=1$ and $T_{1}=x$. Their $L^{2}$ norms are

$$
\left\|T_{k}\right\|_{w}^{2}=c_{k} \frac{\pi}{2}
$$

where $c_{k}=2$ for $k=0$ and $c_{k}=1$ for $k>0$.

\section{Legendre Polynomials}

Legendre polynomials are a special case of Jacobi polynomials for $\alpha=\beta=0$ and satisfy the three-term recursion rule,

$$
L_{k+1}(x)=\frac{2 k+1}{k+1} x L_{k}(x)-\frac{k}{k+1} L_{k-1}(x)
$$

with $L_{0}(x)=1$ and $L_{1}(x)=x$. Their $L^{2}$ norms are

$$
\left\|L_{k}\right\|^{2}=\frac{2}{2 k+1}
$$




\subsubsection{Polynomial Series}

Since the Jacobi polynomials form a basis for $L_{w}^{2}(-1,1)$, any square integrable function, $f(x)$, can be represented as an infinite series in them

$$
f(x)=\sum_{k=0}^{\infty} \hat{f}_{k} P_{k}^{(\alpha, \beta)}(x) .
$$

Furthermore, because the orthogonal characteristic of the basis functions, the coefficients can be found as the projection

$$
\hat{f}_{k}=\frac{\left(f(x), P_{k}^{(\alpha, \beta)}(x)\right)_{w}}{\left\|P_{k}^{(\alpha, \beta)}\right\|_{w}^{2}} .
$$

Introducing the notation $\phi_{k}(x)=P_{k}^{(\alpha, \beta)}(x)$ the truncated series can be described by

$$
f(x)=\sum_{k=0}^{N} \hat{f}_{k} \phi_{k}(x)+\sum_{k=N+1}^{\infty} \hat{f}_{k} \phi_{k}(x)=\sum_{k=0}^{N} \hat{f}_{k} \phi_{k}(x)+\tau,
$$

resulting in a truncation error. Due to the orthogonality of the basis functions, the norm of the truncation error follows

$$
\|\tau\|_{w}^{2}=\sum_{k=N+1}^{\infty}\left|\hat{f}_{k}\right|^{2}\left\|\phi_{k}\right\|_{w}^{2}
$$

It should be noted that $\left\|\phi_{k}\right\|_{w}^{2}$, from (2.39) and (2.41), is constant or algebraically decreasing. Accordingly, the rate of convergence of the approximation depends only on the decay rate of the coefficients (2.43) (like for the Fourier series).

\subsubsection{Polynomial Quadrature}

The quadrature rules are introduced for polynomial basis functions (with respect to an orthogonal function). The quadrature rule which approximates an integral of a function is defined as

$$
\int_{a}^{b} f(x) w(x) d x \approx \sum_{i=0}^{N} w_{i} f\left(x_{i}\right),
$$

where $w_{i}$ and $x_{i}$ are the quadrature weights and nodes. The quadrature rule for Jacobi polynomials satisfies

$$
\sum_{j=0}^{N} \phi_{k}\left(x_{j}\right) \phi_{l}\left(x_{j}\right) w_{j}=\int_{-1}^{1} \phi_{k}(x) \phi_{l}(x) w(x) d x
$$

over the widest possible range of $k$ and $l$. The quadrature rules with the maximum accuracy is the Gauss quadrature. Given a set of nodes $\left\{x_{0}, x_{1}, \ldots, x_{N}\right\}$ the weights 
$\left\{w_{0}, w_{1}, \ldots, w_{N}\right\}$ are calculated to make the formula exact for the polynomials $\left\{1, x, \ldots, x^{N}\right\}$.

$$
w_{i}=\int_{a}^{b} x^{i} w(x) d x \quad i=0,1, \ldots N .
$$

The Gauss integration formula establishes that if $\left\{w_{0}, w_{1}, \ldots, w_{N}\right\}$ are calculated using (2.48) and $\left\{x_{0}, x_{1}, \ldots, x_{N}\right\}$ the zeros of the $(N+1)^{t h}$ polynomial in an orthogonal basis, the quadrature method is exact for any polynomial of degree $\leq 2 N+1$.

The Legendre Gauss quadrature is

$$
\begin{gathered}
x_{i}=\text { zeros of } L_{N+1}(x), \\
w_{i}=\frac{2}{\left(1-x_{i}^{2}\right)\left[L_{N+1}^{\prime}\left(x_{i}\right)\right]^{2}} .
\end{gathered}
$$

The Chebyshev Gauss quadrature reduces to

$$
x_{i}=\text { zeros of } T_{N+1}(x)
$$

which has the analytical expression

$$
\begin{gathered}
x_{i}=\cos \left(\frac{(2 i+1) \pi}{2 N+2}\right) \quad i=0,1, \ldots N, \\
w_{i}=\frac{\pi}{N+1} .
\end{gathered}
$$

Both Gauss quadratures are exact for any polynomial of degree $\leq 2 N+1$ because the zeros of the $(N+1)^{t h}$ polynomial in an orthogonal basis have been used.

To impose boundary conditions, it is useful to define quadrature rules that include the endpoints. The Gauss quadrature rules that include the endpoints $[-1,1]$ are known as the Gauss-Lobatto rules. As the position of the integration nodes has been restricted, the precision of these rules is lower. In fact, the Gauss-Lobatto rules are exact for polynomials of degree $\leq 2 N-1$. Instead of using the roots of the $(N+1)^{t h}$ Chebyshev polynomial, the zeros of the polynomial $\left(1-x^{2}\right) T_{N}^{\prime}(x)$ are the proper choice. The Chebyshev GaussLobatto quadrature reduces to

$$
x_{i}=\text { zeros of }\left(1-x^{2}\right) T_{N}^{\prime}(x),
$$

which has as an analytical expression

$$
x_{i}=\cos \left(\frac{\pi i}{N}\right) \quad i=0,1, \ldots, N
$$


Now the weights are

$$
w_{i}=\left\{\begin{array}{cc}
\frac{\pi}{2 N} & \text { if } i=0 \text { or } N \\
\frac{\pi}{N} & \text { if } i=1, \ldots, N-1 .
\end{array}\right.
$$

Using the quadrature rule (2.47), it is possible to define the discrete inner product for orthogonal polynomial approximations as

$$
(u, v)_{N}=\sum_{i=0}^{N} u\left(x_{i}\right) v\left(x_{i}\right) w_{i} .
$$

It is possible to calculate the norm of the Jacobi polynomials using the discrete inner product. As for the Gauss points, the quadrature is exact for $n \leq N$, so (2.39) and (2.41) are recovered,

$$
\left\|\phi_{n}\right\|_{N}^{2}= \begin{cases}\frac{2}{2 n+1} & \text { Legendre Gauss } \\ \frac{\pi}{2} c_{n} & \text { Chebyshev Gauss }\end{cases}
$$

where $c_{0}=2$ and $c_{n}=1$ for $n>0$.

For the Gauss-Lobatto points, the quadrature is exact for $n<N$, therefore (2.39) is valid. When $n=N$,

$$
\left\|\phi_{n}\right\|_{N}^{2}=\pi \quad \text { Chebyshev Gauss-Lobatto. }
$$

\subsubsection{Orthogonal Polynomial Interpolation}

As it was done for the Fourier series, it is possible to define the polynomial interpolant

$$
I_{N} f(x)=\sum_{k=0}^{N} \tilde{f}_{k} \phi_{k}(x)
$$

that satisfies the interpolation conditions

$$
I_{N} f\left(x_{j}\right)=f\left(x_{j}\right), \quad j=0,1, \ldots, N .
$$

The discrete coefficients $\tilde{f}_{k}$ can be calculated by means of the defined discrete quadrature rules. Furthermore, as the defined formulas are exact for polynomials of degree $\leq 2 N+1$ for Gauss points, it is possible to use the discrete orthogonal projection

$$
\tilde{f}_{n}=\frac{\left(I_{N} f, \phi_{n}\right)_{N}}{\left\|\phi_{n}\right\|_{N}^{2}}=\frac{\left(f, \phi_{n}\right)_{N}}{\left\|\phi_{n}\right\|_{N}^{2}}, \quad n=0,1, \ldots, N
$$


So it is possible to write $(2.60)$ as

$$
\begin{aligned}
I_{N} f(x) & =\sum_{k=0}^{N} \sum_{i=0}^{N} \frac{1}{\left\|\phi_{k}\right\|_{N}^{2}} f\left(x_{i}\right) \phi_{k}\left(x_{i}\right) w_{i} \phi_{k}(x) \\
& =\sum_{i=0}^{N} f\left(x_{i}\right) \sum_{k=0}^{N} \frac{1}{\left\|\phi_{k}\right\|_{N}^{2}} \phi_{k}\left(x_{i}\right) w_{i} \phi_{k}(x) .
\end{aligned}
$$

Furthermore, as interpolation is unique the polynomial interpolants will be often written in the Lagrange form

$$
I_{N} f(x)=\sum_{i=0}^{N} f\left(x_{i}\right) \ell_{i}(x),
$$

where the functions $\ell_{i}(x)$ are the Lagrange interpolating polynomials

$$
\ell_{i}(x)=\prod_{\substack{j=0 \\ i \neq j}}^{N} \frac{\left(x-x_{j}\right)}{\left(x_{i}-x_{j}\right)}
$$

Equations (2.63) and (2.64) are equivalent, through

$$
\ell_{i}(x)=\sum_{k=0}^{N} \frac{1}{\left\|\phi_{k}\right\|_{N}^{2}} \phi_{k}\left(x_{i}\right) w_{i} \phi_{k}(x)
$$

provided that the Lagrange interpolating nodes, $x_{i}, x_{j}$, are the quadrature nodes.

\subsubsection{Polynomial Interpolation Error}

Similarly to the Fourier interpolation, polynomial interpolation is less accurate than series truncation due to aliasing error. This is proved by a procedure similar to the one for the Fourier expansion: relating exact and discrete coefficients and substituting them into the expression of the interpolation. Then it is possible to separate truncation and aliasing errors.

First, the function $f(x)$ in $(2.62)$ is substituted by its polynomial truncated series $(2.44)$

$$
\tilde{f}_{n}=\frac{\left(\sum_{k=0}^{N} \hat{f}_{k} \phi_{k}(x), \phi_{n}\right)_{N}}{\left\|\phi_{n}\right\|_{N}^{2}}+\frac{\left(\sum_{k=N+1}^{\infty} \hat{f}_{k} \phi_{k}(x), \phi_{n}\right)_{N}}{\left\|\phi_{n}\right\|_{N}^{2}}, \quad n=0,1, \ldots, N
$$

and due to the orthogonality of the polynomials

$$
\tilde{f}_{n}=\hat{f}_{n}+\frac{\left(\sum_{k=N+1}^{\infty} \hat{f}_{k} \phi_{k}(x), \phi_{n}\right)_{N}}{\left\|\phi_{n}\right\|_{N}^{2}}, \quad n=0,1, \ldots, N .
$$

The last sum is exactly zero for the exact inner product. However, a quadrature error 
occurs for $k+m>2 N+1$ for Gauss quadrature (or $k+m>2 N-1$ for Gauss-Lobatto) and therefore it is not possible to assure that the last sum will vanish.

Now, replacing the new definition of the interpolation coefficients, (2.68), in the definition of the orthogonal polynomial interpolation (2.60)

$$
I_{N} f(x)=\sum_{n=0}^{N} \hat{f}_{n} \phi_{n}+\sum_{n=0}^{N}\left[\frac{1}{\left\|\phi_{n}\right\|_{N}^{2}}\left(\sum_{k=N+1}^{\infty} \hat{f}_{k} \phi_{k}(x), \phi_{n}\right)_{N}\right] \phi_{n} .
$$

Comparing (2.44) and (2.69) it is possible to see that the first term on the RHS in (2.69) is the truncated series, $P_{N} f$, while the second is, again, the aliasing error, $R_{N} f$,

$$
I_{N} f=P_{N} f+R_{N} f
$$

As it was done for the Fourier approach, it is possible to compare (2.69) with the complete orthogonal polynomial series (2.44) to see that

$$
\begin{aligned}
f(x)-I_{N} f(x) & =\sum_{n=N+1}^{\infty} \hat{f}_{n} \phi_{n}+\sum_{n=0}^{N}\left[\frac{1}{\left\|\phi_{n}\right\|_{N}^{2}}\left(\sum_{k=N+1}^{\infty} \hat{f}_{k} \phi_{k}(x), \phi_{n}\right)_{N}\right] \phi_{n} \\
& =\tau+R_{N} f .
\end{aligned}
$$

Due to the orthogonality of the polynomials, the truncation error, $\tau$, and the aliasing error, $R_{N} f$, are orthogonal so

$$
\left\|f-I_{N} f\right\|^{2}=\left\|f-P_{N} f\right\|^{2}+\left\|R_{N} f\right\|^{2}=\|\tau\|^{2}+\left\|R_{N} f\right\|^{2},
$$

which means that the orthogonal polynomial interpolation is never better than the orthogonal polynomial series. Finally, from (2.71), as Legendre and Chebyshev polynomials are bounded by one, the following expression is valid

$$
\left|f(x)-I_{N} f(x)\right| \leq 2\left\{\sum_{k=N+1}^{\infty}\left|\hat{f}_{k}\right|\right\} .
$$

Moreover, as a consequence of spectral accuracy, in the asymptotic range and for sufficiently smooth functions, $\left|\hat{f}_{k+1}\right|<<\left|\hat{f}_{k}\right|$, therefore

$$
\left|f(x)-I_{N} f(x)\right|=\mathcal{O}\left(\hat{f}_{N+1}\right) .
$$

This result is very important as it permits to estimate the interpolation error as the coefficient of the $N+1$ polynomial. 


\subsubsection{Polynomial Interpolation based Differentiation}

As in Fourier approach, it is possible to approximate the derivative of a function by differentiating its interpolant. Taking the derivative of (2.60),

$$
\left(I_{N} f\right)^{\prime}=\sum_{k=0}^{N} \tilde{f}_{k} \phi_{k}^{\prime}(x) .
$$

However, the result of this operation is more complicated than in the Fourier series. As a result, it is required to go into more details in order to get estimates of the accuracy of the derivatives of the interpolations. For a complete derivation, see [Canuto 2006].

For the Chebyshev interpolation, with Gauss or Gauss-Lobatto nodes, if the approximated function $u$ has a regularity ellipse whose sum of semi-axes equals $e^{\eta}>1$, the following estimates hold in the $L_{w}^{2}$ norm

$$
\left\|u^{(l)}-\left(I_{N} u\right)^{(l)}\right\|_{L_{w}^{2}} \leq C N^{2 l} e^{-N \eta}
$$

while in the maximum norm

$$
\left\|u^{(l)}-\left(I_{N} u\right)^{(l)}\right\|_{L_{\infty}} \leq C N^{1 / 2} N^{2 l} e^{-N \eta} .
$$

Notice that the maximum norm is defined as $\|u\|_{L_{\infty}}=\sup _{-1 \leq x \leq 1}|u(x)|$. Similar to what happened in Fourier approach, the approximation of the derivative is worse than the approximation of the function by an algebraic factor of $N^{2 l}$. As explained before, the convergence for the Legendre series is identical but for algebraic factors.

\subsection{Approximation of PDE Solutions}

Previously it was shown how to approximate functions, derivatives and integrals with high order orthogonal polynomials. In this section it is shown how these methods can finally be used to solve partial differential equations (PDE).

Any PDE with time dependency can be represented by

$$
\frac{\partial u}{\partial t}-\mathcal{L}(u)=f(x)
$$

where $\mathcal{L}$ is the spatial partial differential operator and $f(x)$ a source term. An approximation of the solution can be determined considering that the solution and its derivatives can be expressed as a truncated expansion by a sum of the basis functions:

$$
u \approx u^{N}=\sum_{k=1}^{N} a_{k} \phi_{k} .
$$


Accordingly, the choice of the basis functions, $\phi_{k}$ is the essential part of solving the PDE. The previously introduced spectral methods are characterized by the use of a high order orthogonal basis. In particular Fourier, Chebyshev and Legendre basis were shown. While Fourier bases are only applicable for periodic problems, Chebyshev bases and Legendre polynomials can be used on finite domains. After substituting the truncated approximation (2.79) into the PDE (2.78), the equation is no longer fulfilled,

$$
R=\frac{\partial u^{N}}{\partial t}-\mathcal{L}\left(u^{N}\right)-f^{N} \neq 0
$$

and a residual $R$ remains.

The methods how to approximate PDE solutions can be classified in two categories depending on the way how they minimize the residual. In the case of a weak formulation, the residual is treated in an integral way. The strong formulation forces the residual to be zero at a set of points.

Collocation methods are an example for strong formulations, while Galerkin type methods usually refer to weak formulations.

Collocation methods are based on the strong form of the equation (2.80). Furthermore, equation (2.80) has to be satisfied at certain number of nodes $x_{k}$, which determines the degrees of freedom.

$$
R\left(x_{k}\right)=0 .
$$

Collocation methods are easy to derive and to implement for a wide class of problems. The main drawback of this methodology is the lack of formal guidance to derive a stable approximation and the implementation of boundary conditions. The latter makes collocation methods difficult to apply in complex geometries.

Minimizing the residual leads to

$$
\int_{\Omega} R \psi_{k} d \Omega=0
$$

where the $\psi_{k}$ are called test functions. Galerkin methods are the most common class of spectral methods that are derived from the weak form of the equations. In a Galerkin method, the basis for the test functions, $\psi_{k}$ is chosen from the same set like the basis functions in order to approximate the solution (2.79).

Galerkin methods can be classified into modal or nodal approaches. In the modal Galerkin approach the integrals are solved analytically, which usually results in more accurate solutions, depending on the problem. The drawback is that they are harder to derive and more complex to implement. In contrast, nodal Galerkin methods approximate the integrals by means of quadrature. Accordingly they are less accurate but easier to implement. For various equations, e.g. systems of conservation laws, nodal Galerkin methods are generally easier to implement than collocation schemes, in particular for multiple space dimensions. In order to avoid confusion, it should be noted that in the literature the terms 
modal and nodal may refer to the basis used for numerical integrations. In particular, for a weak formulation they may refer to the modal basis (e.g. Legendre polynomial) or nodal basis (e.g. Lagrange polynomials) [Karniadakis 2013, Hesthaven 2008]. In the former, the unknowns are the coefficients of the modes whilst in the later these coefficients relate to physical nodes in the computational mesh. Both methods are equivalent and related through a Vandermonde matrix, further details may be found in [Karniadakis 2013, Hesthaven 2008].

In this work the Discontinuous Galerkin Spectral Element Method is used for the weak formulation. This method is introduced in more detail in the following.

\subsection{Discontinuous Galerkin Spectral Element Method}

Discontinuous Galerkin methods were first developed [Reed 1973] to solve conservation laws of the form

$$
u_{t}+\nabla \cdot \mathbf{f}=0
$$

A particular nodal variant of the discontinuous Galerkin technique is used here, the Discontinuous Galerkin Spectral Element Method (DGSEM), see Kopriva [Kopriva 2009], which solves Eq. 2.83 in general three-dimensional geometries in which the domain $\Omega$ is divided into $k$ non-overlapping quadrilateral elements $\Omega^{k}$. In this work, the approximation is restricted, without loss of generality, to two-dimensional problems. Each element in the domain is mapped individually onto a unit square by an iso-parametric transformation. This mapping between the unit square and the physical space is described generically by $\mathbf{x}=\mathbf{r}(\xi, \eta)$ where $\xi, \eta$ are the computational coordinates on the unit square. For a complete derivation of the DGSEM method, the reader is referred to Kopriva [Kopriva 2009].

On each element the solution is approximated by a series of orthogonal (w.r.t. the $L_{2}$ inner product) polynomials $P_{N}$ of degree $N$, for more information see Canuto et al. [Canuto 2010]. As a basis for this approximation, a set of Lagrange interpolating polynomials $l_{j}(\xi), j=0, \ldots, N$ is used and can be written as

$$
l_{j}(\xi)=\prod_{\substack{i=j \\ i \neq j}}^{N} \frac{\xi-\xi_{i}}{\xi_{j}-\xi_{i}}
$$

The nodal points $\xi_{i}$, which represent the grid points of the scheme, are chosen to be the nodes of the Legendre-Gauss quadrature. Multiple space dimensions are spanned by tensor products of these polynomials, so it can be written $P_{N, N}=P_{N} \times P_{N}$. For simplicity, the same polynomial order in each direction is taken in most of the cases, though this is not required in practice. As a matter of fact, different polynomial orders in each direction are used in other sections of this paper. From this point onwards and to simplify the notation, it is considered that no mapping is performed, i.e. only the reference element 


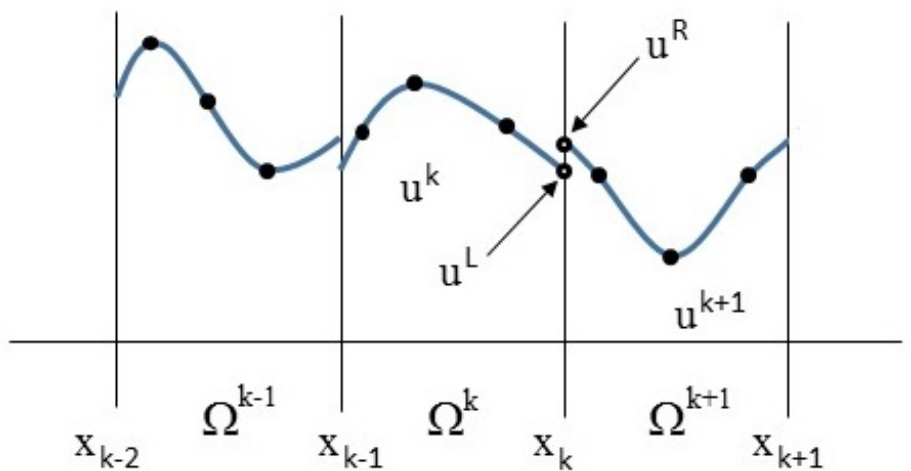

Figure 2.4: Subdivision of the physical domain into k elements. The DGSEM does not enforce continuity at the element boundaries. A Riemann problem is solved to determine the value of the fluxes at the boundaries.

in the computational space is used. In two dimensions, the spectral element method approximates the solution and the fluxes element-by-element by the polynomials

$$
u^{N}(\xi, \eta)=\sum_{\mu, \nu=0}^{N} u_{\mu, \nu}^{N} \phi_{\mu, \nu}, \quad \mathbf{f}^{N}(\xi, \eta)=\sum_{\mu, \nu=0}^{N} \mathbf{f}_{\mu, \nu}^{N} \phi_{\mu, \nu},
$$

where $\phi_{\mu, \nu}=\ell_{\mu}(\xi) \ell_{\nu}(\eta)$. The nodal (grid point) values of the fluxes are computed from the grid point values of the solution, i.e. $\mathbf{f}_{\mu, \nu}^{N}=\mathbf{f}\left(u_{\mu, \nu}^{N}\right)$. Note that $u_{\mu, \nu}^{N}$ is not the nodal value of $u(\xi, \eta)$, but the result of solving the discretized PDE. Therefore the following can be distinguished

$$
u^{N}(\xi, \eta)=\sum_{\mu, \nu=0}^{N} u_{\mu, \nu}^{N} \phi_{\mu, \nu}, \quad \text { and } \quad I_{N} u(\xi, \eta)=\sum_{\mu, \nu=0}^{N} u_{\mu, \nu} \phi_{\mu, \nu}
$$

The former is the solution of the discretized PDE while the latter is the spectral interpolation of the exact solution of the PDE. The same applies to the fluxes

$$
\mathbf{f}^{N}(\xi, \eta)=\sum_{\mu, \nu=0}^{N} \mathbf{f}_{\mu, \nu}^{N} \phi_{\mu, \nu}, \quad \text { and } \quad I_{N} \mathbf{f}(\xi, \eta)=\sum_{\mu, \nu=0}^{N} \mathbf{f}_{\mu, \nu} \phi_{\mu, \nu}
$$

As generally imposed in variational formulations, the PDE residual is required to be orthogonal to the approximation space locally within an element. Thus,

$$
\left(u_{t}^{N}, \phi_{i, j}\right)+\left(\nabla \cdot \mathbf{f}^{N}, \phi_{i, j}\right)=0 \quad i, j=0,1, \ldots, N
$$

where $(a, b)$ represents the usual $L_{2}(\Omega)$ inner product. Integration by parts of Eq. 2.88 
gives

$$
\left(u_{t}^{N}, \phi_{i, j}\right)+\sum_{e \in \partial \Omega} \int_{e} \mathbf{f}^{N} \cdot \mathbf{n} \phi_{i, j} d S-\left(\mathbf{f}^{N}, \nabla \phi_{i, j}\right)=0 \quad i, j=0,1, \ldots, N
$$

where $\mathbf{n}$ is the outward normal unit vector, $\partial \Omega$ represents the boundary of the element and the summation is extended over the edges $e$ of $\partial \Omega$.

To solve this set of equations, the integrals are replaced by Legendre-Gauss quadratures, which in two-dimensional rectangular domains become

$$
\int_{-1}^{1} \int_{-1}^{1} v(\xi, \eta) d \xi d \eta=\sum_{i, j=0}^{N} v\left(\xi_{i}, \eta_{j}\right) w_{i} w_{j} \quad \forall v \in P_{2 N+1,2 N+1}
$$

where $\xi_{i}, \eta_{j}$ are the nodes of the Legendre-Gauss quadrature and $w_{i}, w_{j}$ the corresponding weights. This replacement is exact provided that the element sides are straight. Finally, substituting Eq. 2.85 into Eq. 2.89, taking into account Eq. 2.90 and the discrete orthogonality of the Lagrange interpolating polynomials (Canuto et al. [Canuto 2010]) yields

$$
u_{t_{i, j}}^{N} w_{i} w_{j}+\sum_{e \in \partial \Omega} \int_{e}^{N} \mathbf{f}^{N} \cdot \mathbf{n} \phi_{i, j} d S-\sum_{\mu, \nu=0}^{N} \mathbf{f}_{\mu, \nu}^{N} \cdot \nabla \phi_{i, j} w_{\mu} w_{\nu}=0 \quad i, j=0,1, \ldots, N .
$$

In Eq. 2.91, $\sum_{e \in \partial \Omega} \int_{e}^{N} \mathbf{f}^{N} \cdot \mathbf{n} \phi_{i, j} d S$ is the sum of all the integrals over all the edges of the element approximated by quadrature. The boundary term can be written as follows

$$
\begin{aligned}
\sum_{e \in \partial \Omega} \int_{e}^{N} \mathbf{f}^{N} \cdot \mathbf{n} \phi_{i, j} d S & =\mathbf{f}^{N}\left(1, \eta_{j}\right) \phi_{i, j}\left(1, \eta_{j}\right) w_{j}-\mathbf{f}^{N}\left(-1, \eta_{j}\right) \phi_{i, j}\left(-1, \eta_{j}\right) w_{j} \\
& +\mathbf{f}^{N}\left(\xi_{i}, 1\right) \phi_{i, j}\left(\xi_{i}, 1\right) w_{i}-\mathbf{f}^{N}\left(\xi_{i},-1\right) \phi_{i, j}\left(\xi_{i},-1\right) w_{i}
\end{aligned}
$$

Finally, the discrete partial differential operator for each element can be defined as

$$
\mathcal{R}^{N}(u)=\sum_{e \in \partial \Omega} \int_{e}^{N} I_{N} \mathbf{f}^{*} \cdot \mathbf{n} \phi_{i, j} d S-\sum_{\mu, \nu=0}^{N} \mathbf{f}_{\mu, \nu} \cdot \nabla \phi_{i, j} w_{\mu} w_{\nu} \quad i, j=0,1, \ldots, N,
$$

where $\mathbf{f}^{*}$ is the approximation of the Riemann problem (e.g. Roe's method is selected in this work) [Toro 1997]. Having obtained a suitable discrete expression for each elemental contribution, it suffices to sum over all elements in the mesh and apply the boundary conditions weakly to finalize the DGSEM method, see details in [Kopriva 2009].

The $\tau$-estimation procedure requires to interpolate the solution from a fine (using a high polynomial order) to a coarse grid (using a low polynomial order). Since the DGSEM works with the values of polynomial expansions from a set of nodes, the interpolant from 
order $P$ to $N$ is

$$
I_{P}^{N} \mathbf{f}^{P}\left(\xi_{i}, \eta_{j}\right)=\sum_{\mu, \nu=0}^{P} \mathbf{f}\left(\xi_{\mu}, \eta_{\nu}\right) \phi_{\mu, \nu}\left(\xi_{i}, \eta_{j}\right), \quad i, j=0, \ldots, N
$$

where $\left(\xi_{i}, \eta_{j}\right)$ are the $(N+1) \times(N+1)$ Gauss-Legendre nodal points of order $N$ and $\left(\xi_{\mu}, \eta_{\nu}\right)$ the $(P+1) \times(P+1)$ Gauss-Legendre nodal points of order $P . I_{P}^{N} f^{P}$ is the polynomial of order $N$ whose values in the Gauss-Legendre nodes of order $N$ match $\mathbf{f}^{P}$. To apply the discrete operator $\mathcal{R}^{N}$ to a solution of different order $u^{P}$, it is necessary to evaluate this solution at the Gauss-Legendre nodes of order $N$, i.e. to interpolate to a lower polynomial order coarse grid. For compactness, the notation in this work omits the interpolant such that $\mathcal{R}^{N} u^{P}=\mathcal{R}^{N} I_{P}^{N} u^{P}$.

\subsection{Implementation and Verification}

The previously introduced nodal, explicit 2D high order DG code based on the DGSEM formulation [Kopriva 2009] has been used to provide results for compressible flows. To increase the flexibility of the solver a mortar element method is used to couple the element faces if hanging nodes or elements with varying polynomial orders are used in the computational mesh. Temporal advancement is provided by a 3rd Order Runge Kutta scheme. The code uses OpenMP parallelization with shared memory.

Information about scalability in terms of error and computational cost can be found in Annex A. Furthermore, a comparison of the implemented compressible solver to an incompressible approach will be shown. This comparison aims to show the limitation of the compressible solver in incompressible flow regimes. 


\section{Error Estimation}

\section{Contents}

3.1 Definitions of Errors . . . . . . . . . . . . . . . . . . 33

3.2 DGSEM Error Analysis . . . . . . . . . . . . . . . . . 34

3.2.1 Discretization Error . . . . . . . . . . . . . . 34

3.2 .2 Iteration Error . . . . . . . . . . . . . . . . . . 35

3.2 .3 Truncation Error . . . . . . . . . . . . . . . 35

3.2.4 Truncation Error Limitations . . . . . . . . . . . . . . 37

3.2.5 Truncation Error and Functionals . . . . . . . . . . . . . 38

3.3 Error Estimation . . . . . . . . . . . . . . . . . . . 39

In this chapter errors that are of importance for an adaptation process, are explained. Furthermore, it will be detailed how they can serve as an refinement criteria in an adaptation process. As stated in the introduction, one of the main difficulties when dealing with local mesh adaptation is to locate the flow regions that. when adapted, will improve the numerical solution.

On the one hand, a good refinement criterion will generate a mesh with minimum error for a given number of degrees of freedom. On the other hand, it is crucial that the computational cost of the refinement criterion is cheap such that the whole adaptation process will be efficient.

In this chapter definitions relevant to the adaptation process will be given. In the first part general errors that occur in context of numerical simulations are explained. Following this, information about how to obtain and determine these errors within the proposed Discontinuous Spectral Element Method of chapter 2 is given. This is a review of the theory given by Rubio in his work, more details can be found in [Rubio 2015].

Finally, in the third part, an efficient estimation approach for the truncation error will be explained. This will be an essential part for the adaptation algorithm theory which will be explained in the next chapters.

To determine these estimations, a posteriori error estimation methods will be used in this chapter. A posteriori methods require the computation of a converged approximate solution to compute the estimation of the error. It will be shown that this requirement can be relaxed for the truncation error and isolated truncation error estimations. This approximate solution is obtained by solving the same problem with a higher polynomial 
order on each element. Then, the error can be estimated for all polynomial orders lower than specified, e.g. $\tau_{P}^{N}$ is the truncation error estimation using a fine simulation with order $P$ to estimate the coarse error with polynomial order $N$, when $N<P$.

First, the spatial partial differential operator is introduced

$$
\mathcal{R}(u)=\mathcal{L}(u)-f=0 .
$$

$\mathcal{L}(u)$ can be any spatial partial differential operator (advection equation, diffusion equation, Navier Stokes equations...) while $f$ accounts for the source terms and the boundary conditions. Furthermore, the discrete spatial partial differential operator is introduced

$$
\mathcal{R}^{N}(u)=\mathcal{L}^{N}(u)-f^{N}=0 .
$$

The superscript $N$ means that the spatial partial differential operator has been discretized with a spectral method of order $N$.

The coupling between the different elements introduces additional complications in the DGSEM and therefore two different definitions arise. The discrete partial differential operator for each element is defined as

$$
\mathcal{R}^{N}(u)=\left.I_{N} f^{*} \ell_{j}\right|_{-1} ^{1}-\sum_{i=0}^{N} f_{i} \ell_{j}^{\prime}\left(x_{i}\right) w_{i}, \quad j=0,1, \ldots, N
$$

and the isolated discrete partial differential operator

$$
\hat{\mathcal{R}}^{N}(u)=\left.I_{N} f \ell_{j}\right|_{-1} ^{1}-\sum_{i=0}^{N} f_{i} \ell_{j}^{\prime}\left(x_{i}\right) w_{i}, \quad j=0,1, \ldots, N
$$

As can be seen, the difference between (3.3) and (3.4) is the use of the solution of the Riemann problem, $I_{N} f^{*}$, as numerical flux. While in the former equation the values at the interfaces of the element are substituted by the Riemann flux, $I_{N} f^{*}$, in the latter the flux is calculated using the interior values $I_{N} f$. This change deactivates the communication between the elements and also between the elements and the boundaries. The generalization of (3.3-3.4) to two dimensional geometries results in (see [Rubio 2015] for more details)

$$
\mathcal{R}^{N}(u)=\sum_{e \in \partial \Omega} \int_{e}^{N} I_{N} \mathbf{f}^{*} \cdot \mathbf{n} \phi_{i, j} d S-\sum_{\mu, \nu=0}^{N} \mathbf{f}_{\mu, \nu} \cdot \nabla \phi_{i, j} w_{\mu} w_{\nu} \quad i, j=0,1, \ldots, N,
$$

for the discrete partial differential operator and

$$
\hat{\mathcal{R}}^{N}(u)=\sum_{e \in \partial \Omega} \int_{e}^{N} I_{N} \mathbf{f} \cdot \mathbf{n} \phi_{i, j} d S-\sum_{\mu, \nu=0}^{N} \mathbf{f}_{\mu, \nu} \cdot \nabla \phi_{i, j} w_{\mu} w_{\nu} \quad i, j=0,1, \ldots, N,
$$


for the isolated discrete partial differential operator.

\subsection{Definitions of Errors}

In this section the errors that are relevant for an adaptation process will be presented. Accordingly discretisation, interpolation, iteration and truncation error are explained in detail.

\section{Discretization Error}

The discretization error is defined as the difference between the exact solution of the problem, $u(x)$, and the approximate solution, $u^{N}(x)$, and serves as an indicator for the quality of the solution

$$
\epsilon^{N}=u-u^{N}
$$

\section{Interpolation Error}

The interpolation error is the difference between a function $u(x)$, and its polynomial interpolant, $I_{N} u(x)$, where the function usually describes the solution of a problem

$$
\varepsilon_{u}^{N}=u-I_{N} u
$$

\section{Iteration Error}

The iteration error is the difference between the steady, time-converged approximate solution, $u^{N}$, and the current approximation of the solution (not time-converged), $\tilde{u}^{N}$,

$$
\epsilon_{i t}^{N}=u^{N}-\tilde{u}^{N}
$$

The iteration error is directly related to the residual of the time-iterative method (eg. Runge-Kutta) that is used to solve the set of discrete partial differential equations

$$
\mathcal{R}^{N}\left(\tilde{u}^{N}\right)=\mathcal{R}^{N}\left(u^{N}-\epsilon_{i t}^{N}\right)=\mathcal{R}^{N}\left(u^{N}\right)-\left.\frac{\partial \mathcal{R}^{N}}{\partial u^{N}}\right|_{u^{N}} \epsilon_{i t}^{N}+\mathcal{O}\left(\epsilon_{i t}^{N}\right)^{2} .
$$

\section{Truncation Error}

The truncation error is defined as the difference between the discrete operator applied to the exact solution and the exact operator applied to the exact solution,

$$
\tau^{N}=\mathcal{R}^{N}(u)-\mathcal{R}(u)
$$

When $u(x)$ is the steady exact solution, the exact operator vanishes such that $\mathcal{R}(u)=0$, and it remains

$$
\tau^{N}=\mathcal{R}^{N}(u) .
$$


The assumption of $u(x)$ being the steady exact solution of the problem $\mathcal{R}(u)=0$ means (3.12) is only valid for steady exact solutions. In this way the truncation error (3.12) can be seen as a quality measure for the spatial discretization to solve this steady problem. Temporal evolutions are considered as iterations, and are only a mathematical artifice to obtain the steady solution of the problem.

Furthermore, all errors in this paper are given in the infinity norm, which is defined as

$$
\|u\|_{L_{\infty}}=\sup _{-1 \leq x \leq 1}|u(x)|
$$

This notation is simplified to $\|u\|_{\infty} \equiv\|u\|_{L_{\infty}}$.

\subsection{DGSEM Error Analysis}

In this section the error analysis is explained in context of the DGSEM approach of chapter 2 .

\subsubsection{Discretization Error}

The discretization error is the difference between the exact solution of the problem, $u$, and the approximate solution, $u^{N}$,

$$
\epsilon^{N} \equiv u-u^{N}
$$

In the asymptotic range, for sufficiently smooth functions, it can be assumed that the convergence of this error is spectral (or exponential). This means that for a fixed size $h_{k}$ of the elements, the error on each element $\Omega_{k}$ with the polynomial order $N_{k}$ is bounded (in the $L_{\infty}$ norm) by

$$
\left\|\epsilon_{k}^{N}\right\|_{L_{\infty}} \leq \sum_{k=1}^{K} C_{k} \exp \left(-\eta_{k} N_{k}\right),
$$

where $C_{k}$ and $\eta_{k}$ are constants that depend on the smoothness of the functions (see Canuto et al. [Canuto 2010] or Hesthaven and Warburton [Hesthaven 2008]) and $K$ is the total number of elements. From Eq. 3.15 it may be conducted that, in order to obtain an accurate solution, it is not only necessary to properly resolve the element $k$, but also the surrounding neighbours. In this work the $L_{\infty}$ norm is considered as the maximum over all equations.

For several dimensions, a "tensor-product"-type error bound is valid for the discretization error

$$
\left\|\epsilon_{k}^{N}\right\|_{L_{\infty}} \leq \sum_{k=1}^{K} \sum_{i=1}^{N_{d i m}} C_{i k} \exp \left(-\eta_{i k} N_{i k}\right),
$$

where $N_{\text {dim }}$ is the number of spatial dimensions of the problem. See [Rubio 2014, Georgoulis 2003] for a more detailed explanation of anisotropic error bounds in discontinuous Galerkin methods. 


\subsubsection{Iteration Error}

The iteration error is defined as the difference between the steady, converged approximate solution $u^{N}$ and the current approximation of the solution (not-converged) $\tilde{u}^{N}$,

$$
\epsilon_{i t}^{N} \equiv u^{N}-\tilde{u}^{N}
$$

The iteration error is directly related to the residual of the iterative method, e.g. RungeKutta in a pseudo time iterative procedure, used to advance the solution of the discrete set of equations in time. Indeed, this can be seen using Taylor series:

$$
\mathcal{R}^{N}\left(\tilde{u}^{N}\right)=\mathcal{R}^{N}\left(u^{N}-\epsilon_{i t}^{N}\right)=\underline{\mathcal{R}^{N}}\left(u^{N}\right)-\left.\frac{\partial \mathcal{R}}{\partial u^{N}}\right|_{u^{N}} \epsilon_{i t}^{N}+\mathcal{O}\left(\epsilon_{i t}^{N}\right)^{2},
$$

where the iteration error is clearly linked to the residual of the discrete operator.

\subsubsection{Truncation Error}

In this section the truncation error in the DGSEM is analyzed. First, it is shown that the truncation error includes an interior and an interfacial contribution (or volume and surface, respectively). Furthermore, it is shown how to decouple both contributions, which results in the definition of the isolated truncation error. Second, the connections of the truncation error to the discretization and interpolation error are established. Third, the behavior of the truncation error for problems with anisotropic solutions is studied.

\subsubsection{Isolated Truncation Error}

The DGSEM allows a straightforward separation between interior and interfacial contributions to the truncation error, given as

$$
\tau^{N}=\mathcal{R}_{\partial \Omega}^{N}(u)+\mathcal{R}_{\Omega}^{N}(u),
$$

where $\mathcal{R}_{\partial \Omega}^{N}(u)$ is the interfacial discretization and $\mathcal{R}_{\Omega}^{N}(u)$ is the interior discretization. Furthermore, the interior contribution can be calculated using the isolated discrete partial differential operator (3.4),

$$
\hat{\mathcal{R}}^{N}(u)=\mathcal{R}_{\Omega}^{N}(u)
$$

From (3.20) the isolated truncation error is defined as the result of the isolated discrete partial differential operator applied to the exact solution,

$$
\hat{\tau}^{N}=\hat{\mathcal{R}}^{N}(u) .
$$


The difference between the truncation error and the isolated truncation error is the interfacial contribution to the truncation error,

$$
\tau^{N}-\hat{\tau}^{N}=\mathcal{R}_{\partial \Omega}^{N}(u)
$$

Further details and a proof can be found in [Rubio 2015].

\subsubsection{Error Dependencies}

The truncation error, Eq. 3.12, and the discretization error, Eq. 3.14, are linked through the Discretization Error Transport Equation (DETE) equation [Roy 2010]. This equation can be derived by substituting the definition of the discretization error, Eq. 3.14, into the definition of the truncation error, Eq. 3.12, and by expanding using Taylor series, to obtain

$$
\left.\tau^{N} \approx \frac{\partial \mathcal{R}^{N}}{\partial u^{N}}\right|_{u^{N}} \epsilon^{N}
$$

Eq. 3.23 is the general expression for $\mathcal{R}$ being a non-linear operator, however a similar result can be written for linear operators

$$
\tau^{N}=\tilde{\mathcal{R}}^{N}\left(\epsilon^{N}\right)
$$

where $\tau^{N}$, acts as the local source for the discretization error. The isolated truncation error, $\hat{\tau}^{N}$, (which is the volumetric contribution of the truncation error) is related to the interpolation error by

$$
\hat{\tau}^{N}=-\left(\nabla \cdot \varepsilon_{\mathbf{f}}^{N}, \phi_{i, j}\right)_{N}
$$

The proof can be found in [Rubio 2015]. Furthermore Rubio gives the following conclusions.

First, both the homogeneous discrete operator for linear problems, $\tilde{\mathcal{R}}^{N}$, and the discrete Jacobian for nonlinear problems, $\left.\frac{\partial \mathcal{R}^{N}}{\partial u^{N}}\right|_{u^{N}}$, establish connections between the elements through the Riemann problem. Equation 3.24 shows that the truncation error in each element is a function of the discretization error in it and, through the Riemann problem, on the discretization error of its neighbors.

Second, the isolated discrete partial differential operator, $\hat{\mathcal{R}}^{N}$, does not include connections between the elements. Therefore by (3.25) the isolated truncation error is a function of the interpolation error inside the element.

Third, although by (3.24-3.25) the truncation error and the isolated truncation error are functions of the discretization and interpolation error, this does not mean that the rate of convergence is the same. For discontinuous Galerkin methods, the truncation error predicts a lower order of accuracy than the actual rate, as a consequence of superconvergence [Zhang 2005]. 


\subsubsection{Anisotropic Behaviour of the Truncation Error}

One of the main topics of interest of this work is the behavior of the truncation error (and also the isolated truncation error) for anisotropic solutions, e.g. boundary layers. In these problems, the difference between the complexity (in the sense of nonlinearity) of the solution in each of the spatial dimensions varies. Without loss of generality, a two dimensional problem where $x$ is the direction of high complexity is supposed. Following (3.16) it is reasonable to describe the discretization, $\epsilon^{N}$, and the interpolation error, $\varepsilon^{N}$, as,

$$
\begin{aligned}
& \epsilon^{N}=\epsilon_{x}^{N}+\epsilon_{y}^{N} \\
& \varepsilon^{N}=\varepsilon_{x}^{N}+\varepsilon_{y}^{N},
\end{aligned}
$$

with $\epsilon_{x}^{N}>>\epsilon_{y}^{N}$ and $\varepsilon_{x}^{N}>>\varepsilon_{y}^{N}$. These two errors contribute to the whole error, which can be decreased by increasing the number of degrees of freedom (DoF) in each spatial direction.

Under these assumptions, the following results are obtained for the truncation error for linear problems

$$
\tau^{N}=\tilde{\mathcal{R}}^{N}\left(\epsilon_{x}^{N}\right)+\mathcal{O}\left(\left.\frac{\partial \mathcal{R}^{N}}{\partial u^{N}}\right|_{u^{N}} \epsilon_{y}^{N}\right)
$$

and for the truncation error for nonlinear problems

$$
\tau^{N}=\left.\frac{\partial \mathcal{R}^{N}}{\partial u^{N}}\right|_{u^{N}} \epsilon_{x}^{N}+\mathcal{O}\left(\max \left(\left(\epsilon^{N}\right)^{2},\left.\frac{\partial \mathcal{R}^{N}}{\partial u^{N}}\right|_{u^{N}} \epsilon_{y}^{N}\right)\right)
$$

Similarly, the following result is obtained for the isolated truncation error

$$
\hat{\tau}^{N}=-\left(\nabla \cdot \varepsilon_{x \mathbf{f}}^{N}, \phi_{i, j}\right)_{N}+\mathcal{O}\left(\varepsilon_{y \mathbf{f}}^{N}\right)
$$

Both the truncation error and the isolated truncation error preserve the anisotropy of the discretization and interpolation errors (see [Rubio 2015] for the proof).

\subsubsection{Truncation Error Limitations}

It is possible to use Eq. 3.23 to find a lower bound for the truncation error. Assuming that the problem is well posed (i.e. invertible Jacobian), the following holds

$$
\left(\left.\frac{\partial \mathcal{R}^{N}}{\partial u^{N}}\right|_{u^{N}}\right)^{-1} \tau^{N} \approx \epsilon^{N}
$$

Taking norms

$$
\left\|\left(\left.\frac{\partial \mathcal{R}^{N}}{\partial u^{N}}\right|_{u^{N}}\right)^{-1} \tau^{N}\right\|_{L_{\infty}} \approx\left\|\epsilon^{N}\right\|_{L_{\infty}}
$$


and using the Cauchy-Schwarz inequality, one can obtain

$$
\left\|\left(\left.\frac{\partial \mathcal{R}^{N}}{\partial u^{N}}\right|_{u^{N}}\right)^{-1}\right\|_{L_{\infty}}\left\|\tau^{N}\right\|_{L_{\infty}} \gtrsim\left\|\epsilon^{N}\right\|_{L_{\infty}}
$$

and rearranging leads to

$$
\left\|\tau^{N}\right\|_{L_{\infty}} \gtrsim \frac{\left\|\epsilon^{N}\right\|_{L_{\infty}}}{\left\|\left(\left.\frac{\partial \mathcal{R}^{N}}{\partial u^{N}}\right|_{u^{N}}\right)^{-1}\right\|_{L_{\infty}}}=\frac{\left\|\epsilon^{N}\right\|_{L_{\infty}}}{\left\|\left(\left.\frac{\partial \mathcal{R}^{N}}{\partial u^{N}}\right|_{u^{N}}\right)\right\|_{L_{\infty}} \kappa\left(\left.\frac{\partial \mathcal{R}^{N}}{\partial u^{N}}\right|_{u^{N}}\right)}
$$

where $\kappa\left(\left.\frac{\partial \mathcal{R}^{N}}{\partial u^{N}}\right|_{u^{N}}\right)=\left\|\left(\left.\frac{\partial \mathcal{R}^{N}}{\partial u^{N}}\right|_{u^{N}}\right)^{-1}\right\|_{L_{\infty}}\left\|\left(\left.\frac{\partial \mathcal{R}^{N}}{\partial u^{N}}\right|_{u^{N}}\right)\right\|_{L_{\infty}}$ denotes the condition number (in the $L_{\infty}$ norm) of the system Jacobian. Eq. 3.33 shows that there exists a direct relationship between truncation and discretisation errors. The latter equation may be used to set a threshold for the truncation error based adaptation.

\subsubsection{Truncation Error and Functionals}

It is possible to show that the truncation error controls functional errors. Let us consider a functional output $\mathcal{J}^{N}(u)$ (e.g. lift or drag coefficient) and expand it using Taylor series about $u$ :

$$
\mathcal{J}^{N}(u)=\mathcal{J}^{N}\left(u^{N}\right)+\left.\frac{\partial \mathcal{J}^{N}}{\partial u^{N}}\right|_{u^{N}}\left(u-u^{N}\right)+\mathcal{O}\left(u-u^{N}\right)^{2} .
$$

Rearranging Eq. (3.34) and taking norms one can obtain

$$
\left\|\mathcal{J}^{N}(u)-\mathcal{J}^{N}\left(u^{N}\right)\right\|_{L_{\infty}} \approx\left\|\left.\frac{\partial \mathcal{J}^{N}}{\partial u^{N}}\right|_{u^{N}}\left(u-u^{N}\right)\right\|_{L_{\infty}} .
$$

Applying the Cauchy-Schwarz inequality to the right hand side of Eq. (3.35) and rearranging, it can be found a lower bound for the discretisation error:

$$
\frac{\left\|\mathcal{J}^{N}(u)-\mathcal{J}^{N}\left(u^{N}\right)\right\|_{L_{\infty}}}{\left\|\left.\frac{\partial \mathcal{J}^{N}}{\partial u^{N}}\right|_{u^{N}}\right\|_{L_{\infty}}} \lesssim\left(u-u^{N}\right)\left\|_{L_{\infty}}=\right\| \epsilon^{N} \|_{L_{\infty}}
$$

Finally combining Eq. (3.33) and Eq. (3.36) it is easy to show that the truncation error controls not only the discretisation error but also the functional error

$$
\left\|\tau^{N}\right\|_{L_{\infty}} \gtrsim \frac{\left\|\epsilon^{N}\right\|_{L_{\infty}}}{\left\|\left(\left.\frac{\partial \mathcal{R}^{N}}{\partial u^{N}}\right|_{u^{N}}\right)^{-1}\right\|_{L_{\infty}}} \gtrsim \frac{\left\|\mathcal{J}^{N}(u)-\mathcal{J}^{N}\left(u^{N}\right)\right\|_{L_{\infty}}}{\left\|\left(\left.\frac{\partial \mathcal{R}^{N}}{\partial u^{N}}\right|_{u^{N}}\right)^{-1}\right\|_{L_{\infty}}\left\|\left.\frac{\partial \mathcal{J}^{N}}{\partial u^{N}}\right|_{u^{N}}\right\|_{L_{\infty}} .}
$$

This brief proof shows that by controlling the truncation error, one can control any derived functional. Therefore an adaption process based on the truncation error should enhance the accuracy of all functionals. 
Additionally, it should be noted that introducing the adjoint vector field $\left.\left(\psi^{N}\right)^{T} \frac{\partial \mathcal{R}^{N}}{\partial u^{N}}\right|_{u^{N}}=$ $\left.\frac{\partial \mathcal{J}^{N}}{\partial u^{N}}\right|_{u^{N}}$ (and using again the Cauchy-Schwarz inequality) one may simplify Eq. (3.37) to

$$
\left\|\tau^{N}\right\|_{L_{\infty}} \gtrsim \frac{\left\|\mathcal{J}^{N}(u)-\mathcal{J}^{N}\left(u^{N}\right)\right\|_{L_{\infty}}}{\left\|\left(\psi^{N}\right)^{T}\right\|_{L_{\infty}} \kappa\left(\left.\frac{\partial \mathcal{R}^{N}}{\partial u^{N}}\right|_{u^{N}}\right)}
$$

where the previously introduced condition number (in the $L_{\infty}$ norm) of the system Jacobian is used again.

Finally, it should be noted that the definition of the truncation error, Eq. 3.12, includes the exact solution of the problem. Since the exact solution is not available in general, an estimation for Eq. 3.12 is necessary. This is covered in the next section.

\subsection{Error Estimation}

In this section the a posteriori and the quasi- $a$ priori methodology to estimate the error is introduced. The former relates to the estimation of the error for a converged solution, while the latter shows an approach to estimate the error based on a solution that is not converged in time.

Since the exact solution, that is required to calculate the truncation error in Eq. 3.12, is not available in general, the $\tau$-estimation method uses an approximate solution instead to estimate the error. This approximate solution is obtained by solving the same problem with a higher polynomial order on each element. Then, the error can be estimated for all polynomial orders lower than specified, e.g. $\tau_{P}^{N}$ is the truncation error estimation using a fine simulation with order $P$ to estimate the coarse error with polynomial order $N$, when $N<P$. This estimation is called a posteriori since it requires a fully converged solution of order $P$.

This approach can be extended to non-converged solutions. The quasi-a priori method permits accurate estimations of the truncation error, $\tau^{N}$, on a fine mesh using a not necessarily converged solution, $\tilde{u}^{P}$. Furthermore, it is possible to derive a correction for this solution which can be incorporated into the method to overcome the lack of temporal convergence.

The expressions to estimate the truncation error were first deduced by Fraysse et al. [Fraysse 2012a] for finite volume schemes and extended later to spectral Chebyshev collocation methods and the DGSEM by Rubio et al. [Rubio 2013, Rubio 2014]. Here the main conclusions for non-converged solutions are summarized. The approximated truncation error becomes

$$
\tau_{P}^{N} \equiv \mathcal{R}^{N}\left(\tilde{u}^{P}\right)-\bar{I}_{P}^{N} \mathcal{R}^{P}\left(\tilde{u}^{P}\right)
$$


Here, $\bar{I}_{P}^{N}$ is the transfer operator of the residual of order $P$ to $N$, defined as

$$
\bar{I}_{P}^{N} \equiv \tilde{\mathcal{R}}^{N} I_{P}^{N}\left(\tilde{\mathcal{R}}^{P}\right)^{-1}
$$

for linear operators. Note that the operator $\mathcal{R}^{N}(u)=\tilde{\mathcal{R}}^{N}(u)+S^{N}$ may be decomposed in a sum of a homogeneous operator $\tilde{\mathcal{R}}^{N}$ that is a function of $u$, and an independent term $S^{N}$, that accounts for the source terms and the value of the boundary conditions. The full derivation can be found in Rubio et al. [Rubio 2014]. The difference between the exact, Eq. 3.11, and the approximate truncation error, Eq. 3.39, is (Rubio et al. [Rubio 2014]):

$$
\tau_{P}^{N}=\tau^{N}-\tilde{\mathcal{R}}^{N}\left(\epsilon^{P}\right)
$$

Likewise, for non-linear operators the transfer operator is defined as

$$
\left.\bar{I}_{P}^{N} \equiv \frac{\partial \mathcal{R}^{N}}{\partial u^{N}}\right|_{u^{N}} I_{P}^{N}\left(\left.\frac{\partial \mathcal{R}^{P}}{\partial u^{P}}\right|_{u^{P}}\right)^{-1}
$$

and the difference between the exact and the approximate truncation error reads

$$
\tau_{P}^{N}=\tau^{N}-\left.\frac{\partial \mathcal{R}^{N}}{\partial u^{N}}\right|_{u^{N}} \epsilon^{P}+\mathcal{O}\left(\epsilon^{P}\right)^{2}+\mathcal{O}\left(\epsilon_{i t}^{P}\right)^{2}
$$

In the case of non-converged solutions, the second term on the right hand side (RHS) of Eq. 3.39 acts as a correction term for the iteration error, $\epsilon_{i t}^{P}$. Indeed, from Eq. 3.18, it can be seen that

$$
\epsilon_{i t} \approx\left(\left.\frac{\partial \mathcal{R}^{P}}{\partial u^{P}}\right|_{u^{P}}\right)^{-1} \mathcal{R}^{N}\left(\tilde{u}^{P}\right)
$$

However, the computation of this correction term is computationally expensive, since it requires the solution of a linear system, therefore a quasi- $a$ priori estimations is performed with and without this correction term. If no correction term is used, i.e. $\bar{I}_{P}^{N} \mathcal{R}^{P}\left(\tilde{u}^{P}\right)$ is not computed, then the difference between the exact and the approximate truncation error becomes

$$
\tau_{P}^{N}=\tau^{N}-\left.\frac{\partial \mathcal{R}^{N}}{\partial u^{N}}\right|_{u^{N}} \epsilon^{P}-\underbrace{\left.\frac{\partial \mathcal{R}^{N}}{\partial u^{N}}\right|_{u^{N}} \epsilon_{i t}^{P}}_{\text {first order iteration error }}+\mathcal{O}\left(\epsilon^{P}\right)^{2}+\mathcal{O}\left(\epsilon_{i t}^{P}\right)^{2} .
$$

It should be noticed that to apply the discrete operator, $\mathcal{R}^{N}$, to a solution of different order, $u^{P}$, it is necessary to evaluate this solution at the Gauss-Legendre nodes of order $N$, i.e. to interpolate to a coarse grid. For compactness, the notation in this work omits the interpolant such that $\mathcal{R}^{N} u^{P}=\mathcal{R}^{N} I_{P}^{N} u^{P}$.

For the a posteriori method (with converged solution), $u^{P}=\tilde{u}^{P}$ is obtained, and the 
second term on the RHS of Eq. 3.39 reduces to:

$$
\tau_{P}^{N}=\mathcal{R}^{N}\left(u^{P}\right)
$$

\section{Isolated Truncation Error}

The isolated truncation error based refinement criterion is also based on the $\tau$-estimation method. Rubio et al. [Rubio 2014] shown that for non-converged solutions and non-linear partial differential operators, the approximated isolated truncation error becomes

$$
\hat{\tau}_{P}^{N} \equiv \hat{\mathcal{R}}^{N}\left(\tilde{u}^{P}\right)-\hat{I}_{P}^{N} \mathcal{R}^{P}\left(\tilde{u}^{P}\right)
$$

Besides $\hat{I}_{P}^{N}$, the transfer operator of the residual from order $P$ to $N$ for the isolated truncation error is,

$$
\left.\hat{I}_{P}^{N} \equiv \frac{\partial \hat{\mathcal{R}}^{N}}{\partial u^{N}}\right|_{\tilde{u}^{P}} I_{P}^{N}\left(\left.\frac{\partial \mathcal{R}^{P}}{\partial u^{P}}\right|_{\tilde{u}^{P}}\right)^{-1}
$$

and the difference between the exact and the approximate truncation error reads

$$
\hat{\tau}_{P}^{N}=\hat{\tau}^{N}-\left.\frac{\partial \hat{\mathcal{R}}^{N}}{\partial u^{N}}\right|_{u} \epsilon^{P}+\mathcal{O}\left(\epsilon_{i t}^{P}\right)^{2} .
$$





\section{Adaptation}

\section{Contents}

4.1 Isotropic Adaptation . . . . . . . . . . . . . . . . . . . . . 44

4.1.1 Truncation / Isolated Truncation Error Adaptation Algorithm . . . 44

$4.1 .2 \quad$ Error Extrapolation . . . . . . . . . . . . . . . . . . . . 46

4.1 .3 Discretisation Error _ . . . . . . . . . . . . . . . . . 48

4.2 Anisotropic Adaptation . . . . . . . . . . . . . . . . . . . . 51

4.2.1 Truncation / Isolated Truncation Error Adaptation Algorithm . . . 51

4.2 .2 Minimization of DoF . . . . . . . . . . . . . . . . . . 51

4.2 .3 Error Extrapolation for minimal DoF . . . . . . . . . . . . . . 52

4.3 Quasi- $a$ priori and quasi- a priori corrected $\tau$-estimation . . . . . . . . 56

4.3.1 Memory Requirements . . . . . . . . . . . . . . . . . . . 56

4.3.2 Theoretical Computational Cost _ . . . . . . . . . . . . 57

In this section the developed adaptation algorithms are explained based on the theory presented in the previous chapters. First, isotropic adaptation algorithms are presented. The common feature of these isotropic schemes is that the error in different coordinate directions are considered together and not independently. Each element is refined entirely, considering the inherent overall error. For p-refinement this results in equally distributed polynomial orders in the different coordinate directions. The isotropic adaptation is introduced using the discretisation error, the truncation error and the isolated truncation error.

In the second part of this chapter the isotropic adaptation procedure will be extended to an anisotropic version which is based on the truncation error estimates. Anisotropic approaches allow to decouple the error in different coordinate directions and enable a higher flexibility of mesh adaptation. In the best case this can lead to a significant reduction of DoF, especially for simulations with directional dominant flow features.

The common philosophy of all the presented algorithms is first to determine one of the errors detailed in chapter 3, obtain their magnitude and use this value as a refinement criterion. All algorithms start with the computation of a solution with a high order polynomial $P$ and use this solution to compute the criterion in all the coarser meshes $N<P$. The aim of all adaptation algorithms is to select the lowest possible polynomial 
order that satisfies the required refinement criterion. Once this new polynomial order is determined, the solution $P$ is interpolated into the new mesh and the simulation restarted.

In the last section it will be explained how the quasi-a priori $\tau$-estimation method can be used to estimate the truncation and the isolated truncation errors with non time converged solutions. The estimation with a non time converged solution allows a significant decrease of the computational cost to attain the same accuracy [Rubio 2014, Kompenhans 2016] which will be shown in more detail in chapter 5 .

Furthermore, the proposed procedures enable the determination of the convergence trend of the error: for example exponential convergence in elements with smooth solutions. Having determined this trend, it is possible to use extrapolation to find higher polynomials that result in a particularly lower error and save computational cost at the same time [Kompenhans 2016].

The following different algorithms are introduced and explained in detail. First, a general isotropic approach is presented for the truncation error, Algorithm 1. This algorithm will be improved by incooporating an error extrapolation scheme, Algorithm 2. Finally, an adaptation algorithm for the discretisation based adaptation is detailed, Algorithm 3 . In the second part, the anisotropic approach based on the truncation error is introduced using error extrapolation, Algorithm 4. This approach is further modified to guarantee a minimal DoF in order to save computational cost, Algorithm 5. In the third part, it is explained how to incorporate a quasi-a priori estimation method to use non-converged solutions for the estimation process. Table 4.1 gives an overview of all presented algorithms.

\begin{tabular}{|l||l|l|l|l|l|}
\hline Algorithm & $\# 1$ & $\# 2$ & $\# 3$ & $\# 4$ & $\# 5$ \\
\hline \hline Isotropic & $\mathrm{X}$ & $\mathrm{X}$ & $\mathrm{X}$ & & \\
\hline Anisotropic & & & & $\mathrm{X}$ & $\mathrm{X}$ \\
\hline Truncation Error & $\mathrm{X}$ & $\mathrm{X}$ & & $\mathrm{X}$ & $\mathrm{X}$ \\
\hline Isolated Truncation Error & $\mathrm{X}$ & $\mathrm{X}$ & & $\mathrm{X}$ & $\mathrm{X}$ \\
\hline Discretisation Error & & & $\mathrm{X}$ & & \\
\hline Extrapolation & & $\mathrm{X}$ & & & \\
\hline Minimal DoF & & & & & $\mathrm{X}$ \\
\hline
\end{tabular}

Table 4.1: Overview of algorithm properties.

\subsection{Isotropic Adaptation}

\subsubsection{Truncation / Isolated Truncation Error Adaptation Algorithm}

The isotropic truncation error based adaptation algorithm relies on the estimate Eq. 3.39 that is based on the $\tau$-estimation method. The complete algorithm is detailed in Algorithm 1. In part A, the simulation is converged until a steady state solution is obtained. Steady state usually is assumed if the solution tolerance is below $10^{-10}$. After this, the truncation error is estimated for all coarser meshes $N<P$. In part $\mathrm{B}$, the new polynomial order is selected. If the estimation fulfills the requirement $\left\|\tau_{P}^{N}\right\|_{L_{\infty}}$ for a certain polynomial order, 
then this polynomial order is set for the new adapted mesh. In part $\mathrm{C}$, the old solution is interpolated to the new mesh and the simulation restarted.

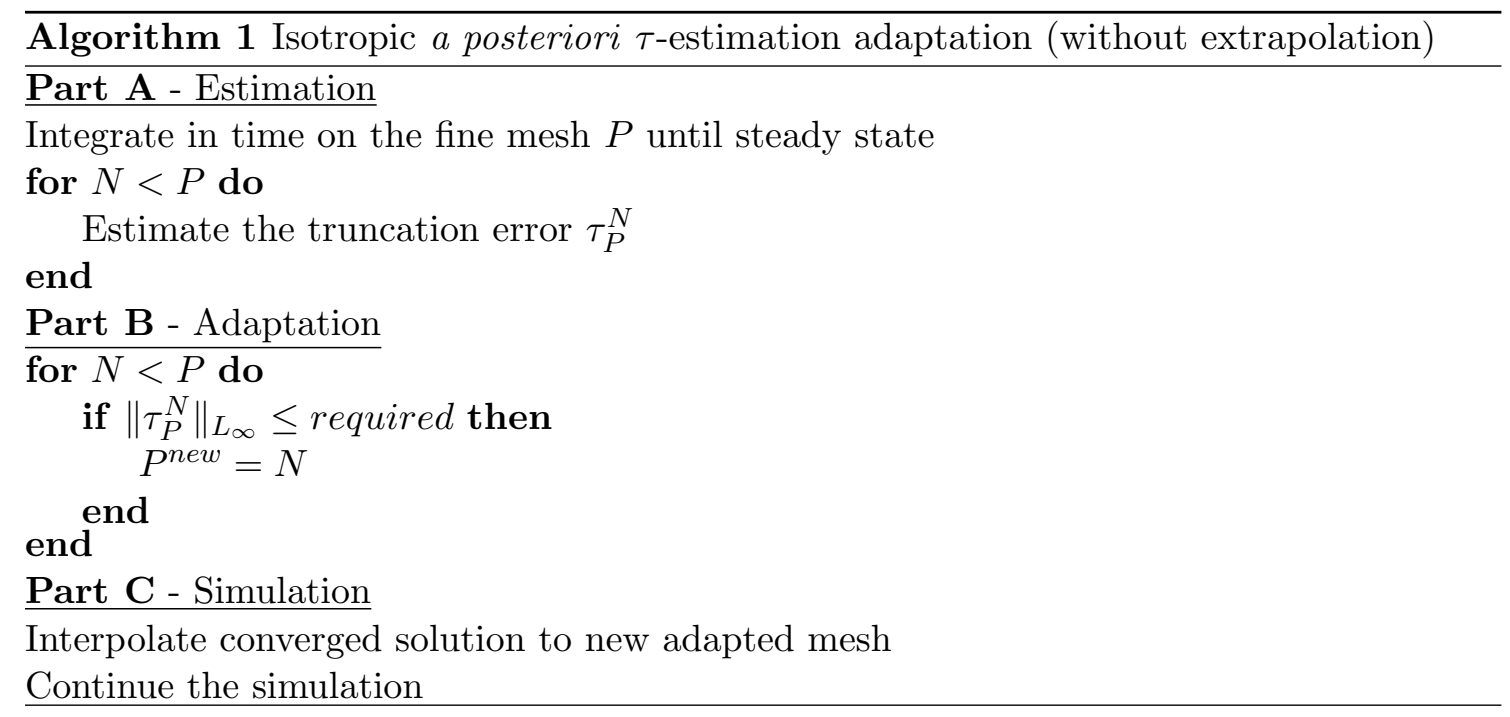

This adaptation algorithm can be modified to use the isolated truncation error by using Eq. 3.47 instead of Eq. 3.39. Using the isolated truncation error leads to different results that likely have better properties. More details are shown in chapter 5 .

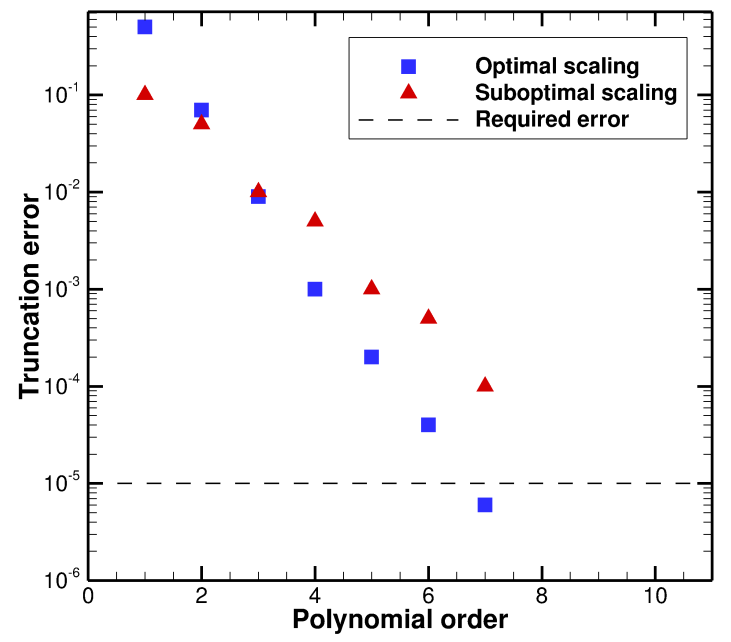

Figure 4.1: Error estimations for two cases: optimal scaling (square) and suboptimal scaling (triangle).

For illustration, Figure 4.1 shows a typical plot of the estimation of the truncation error in $\log$ scale. The error $\left\|\tau_{P}^{N}\right\|_{L_{\infty}}$ in each element is shown as a function of the polynomial order $P$. Now, for a desired error threshold $\tau_{\max }$ (e.g. $10^{-5}$, horizontal dashed line in Figure 4.1), two different possibilities appear depending on whether the finest solution $u^{P}$ has been chosen to be accurate enough. In the first case (squares), the required error can be reached with a polynomial of order $P=7$, which agrees with the estimation already 
performed in the element, this is known as optimal scaling. In the second case (triangles), the required error is not reached by any of the estimates with a lower order than $P$, and a higher polynomial order is needed (i.e. suboptimal scaling).

Suboptimal scaling can occur due to different reasons. In general it can be said that the problem is under-resolved in the related area and the flow features can not be captured in the desired accuracy with the predicted orders. Possibilities for suboptimal scaling could stem from flow features or geometries in that region that are difficult to compute or just a general under-resolution in terms of elements or order.

There are certain approaches to deal with this suboptimal scaling case. The first and most trivial approach would be to re-run the test case using a finer polynomial order $P$ than the previously selected order. Once the new simulation is converged until steady state one could calculate all error estimations and choose the appropriate order, presumed that the error criterion is fulfilled by one of the estimates. If none of the estimates fulfill the criterion an even finer initial simulation has to be considered.

The drawback of this approach certainly is the high computational cost to obtain the initial fine mesh solution. The new attempt unnecessarily prolongs the entire computational process and consequently is undesirable for real industrial problems.

The second approach to deal with a suboptimal scaling is to limit the maximum tolerated polynomial order. In this case the desired error is not guaranteed on the corresponding element with the suboptimal scaling. Nevertheless it can make sense for some cases. First, if the computational resources are limited one might not consider polynomial orders of very high degree which will slow down the computational process. Secondly it makes sense, if one is only interested in a global general view and not necessarily relies on strict error limitations. Especially for elements with critical features, like singularities or points at leading or trailing edges of a profile, it can be difficult to obtain a low error with a reasonable polynomial order. To overcome this drawback, it is more suitable to perform a h-refinement to reduce the error, in particular if the element encompasses singularities.

The third and most efficient approach is to use these existing estimations and extrapolate these error values to obtain the error estimations for the not calculated higher polynomial orders. Consequently it is probable that one of those extrapolated error estimates for the higher polynomial orders will guarantee an error lower than the threshold.

This error extrapolation is permitted since it is assumed that the solution is in the asymptotic range, in which the error decays exponentially. The incorporation of error extrapolation will be explained in detail, in the following section.

\subsubsection{Error Extrapolation}

Different convergence rates were explained in chapter 2. Furthermore, the scaling properties of the truncation error were shown in chapter 3. This part explains how to use this beneficial properties to extend the previously shown algorithm, such that less error estimates are needed and consequently the computational effort is reduced. 
As seen in Figure 4.1 in the previous section, to achieve a required truncation error of $\tau_{\max }=10^{-5}$ a polynomial order of $P=7$ is selected for the optimal scaling case, while the suboptimal scaling case fails to reach the desired error. Accordingly an order of $P>7$ has to be chosen to guarantee this error. To determine the polynomial order, that has to be selected, extrapolation can be used.

In the last case, one may extrapolate the estimate to determine the polynomial order that satisfies the error for the given threshold. Based on Rubio et al. [Rubio 2014], it can be proved that the locally generated truncation error follows an exponential law

$$
\left\|\tau_{k}^{N}\right\| \leq \sum_{i=1}^{N_{d i m}} C_{i k} \exp \left(-\eta_{i k} N_{i k}\right),
$$

where $N_{d i m}$ is the number of spatial dimensions of the problem while $C_{i k}$ and $\eta_{i k}$ are constants that depend on the smoothness of the function, see Canuto et al. [Canuto 2010] or Hesthaven and Warburton [Hesthaven 2008].

It is important to notice that, for two dimensional problems with isotropic solutions and assuming without loss of generality that $N_{x}>N_{y}$, Eq. 4.1 can be approximated by

$$
\left\|\tau_{k}^{N}\right\| \lesssim C_{i k} \exp \left(-\eta_{i k} N_{i k}\right)
$$

where the sub-index $i$ denotes the $x$ or $y$-direction. Taking logarithms in Eq. 4.2, the following asymptotic behavior may be inferred (for the element $k$ ):

$$
\log \left(\left\|\tau_{k}^{N}\right\|_{L_{\infty}}\right) \approx C_{i k}-\eta_{i k} N_{i k}
$$

The two constants, $C_{i k}$ and $\eta_{i k}$, depend on the element and can be easily approximated by least squares fitting in each spatial dimension, see Figure 4.2.

For anisotropic problems (which will be explained in more detail later), Eq. 4.2 is only valid for $N_{x}>>N_{y}$ (i.e. assuming that $N_{x}$ is the spatial dimension with the highest flow complexity, such as large flow gradients). For highly anisotropic problems, even with $N_{x}>N_{y}$ the truncation error may have contributions of both spatial dimensions. Therefore only the values of the truncation error where $N_{x}>>N_{y}$ should be used for the least square fitting.

The selection of suboptimal or suboptimal scaling is described in part B of Algorithm 2 and determines the appropriate polynomial order based on the estimation or the extrapolation. In particular, the desired truncation threshold, $\tau_{\max }$, is set before starting the adaptation procedure.

Finally, in part C, the simulation is continued using the locally p-adapted mesh. Note that the obtained converged solution on the non-adapted mesh can be interpolated to the p-adapted mesh and used as initial condition. In addition, it should be noted that besides the beneficial effect of an increase of the polynomial order in terms of accuracy, also a 


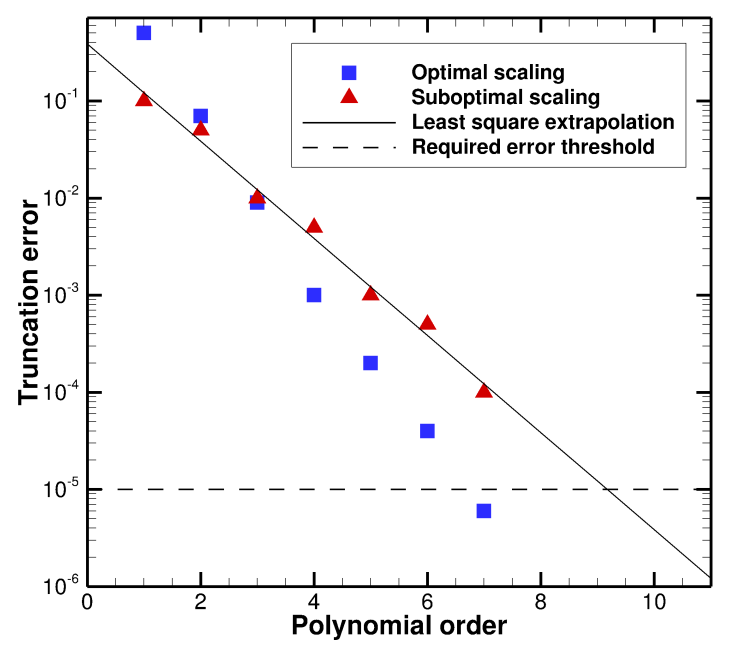

Figure 4.2: Example of the extrapolation process within the adaptation procedure, to determine the polynomial order based on the truncation error: optimal scaling (squares) and suboptimal scaling (triangles). Dashed horizontal line: truncation error threshold and black line: least square extrapolation.

decrease of the polynomial order in certain areas can be expected, which decreases the degrees of freedom and reduces the computational cost.

Assuming that the error estimates for the particular element are in the asymptotic range, a linear least mean square data fit can be used after a logarithmic transformation. The error estimates $\tau_{8}^{1: 7}$ are used to determine the slope of the extrapolant. Once the extrapolant is calculated the intersection with the error threshold line can be determined. Finally, the corresponding polynomial order can be selected.

Algorithm 2 shows the entire approach including error extrapolation.

\subsubsection{Discretisation Error}

The reduction of the discretisation error is an inherent goal of every simulation in order to improve its quality. Accordingly, the usage of the disretisation error as a refinement indicator is a logical consequence.

Different approaches have been made for a discretisation error based adaptation. Persson [Persson 2006] uses a shock capturing strategy for higher order Discontinuous Galerkin approximations based on the discretisation error. The solution on each element is expressed as,

$$
u=\sum_{i=1}^{P} u_{i} \psi_{i},
$$

where $N$ is the polynomial oder and $\psi_{i}$ the basis functions. The truncated expansion of 


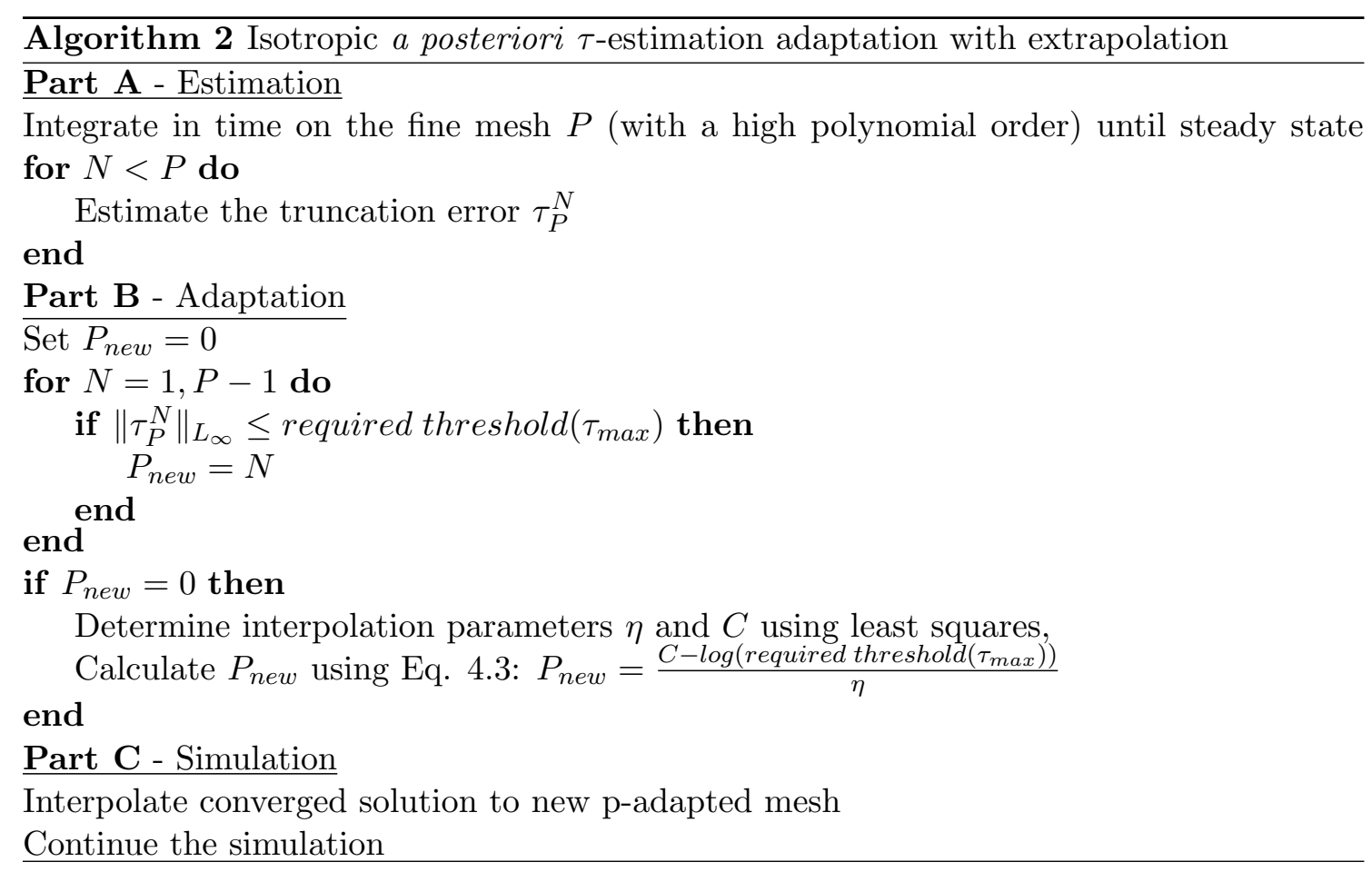

the same solution is only containing terms up to order of p-1

$$
\hat{u}=\hat{u}^{P-1}=\sum_{i=1}^{P-1} u_{i} \psi_{i} .
$$

Accordingly the following smoothness indicator is defined

$$
S_{e}=\frac{(u-\hat{u}, u-\hat{u})_{e}}{(u, u)_{e}}
$$

where $(., .)_{e}$ is the standard inner product in $L_{2}$ for each element e. Therefore, in $1 \mathrm{D}$, the Fourier coefficients would decay at least like $1 / k^{2}$.

While this approach is mainly used to determine the smoothness of a solution in order to detect shocks and conduct a refinement accordingly, similar approaches have been made and used for direct mesh adaptation. Barosan et al. [Barosan 2006] proposed an algorithm using the discretisation error in an adaptation algorithm. His scheme relies on the theory proposed by Mavriplis [Mavriplis 1990]. Further a posteriori estimators were introduced by [Babuska 1981, Berger 1984, Henderson 1994].

The adaptation approach of Barosan relies on the Legendre polynomials and will be explained in more detail in the following.

\subsubsection{Discretisation Error Estimation}

The discretisation error based refinement criterion relies on the theory proposed by Mavriplis [Mavriplis 1990] and was recently used by Barosan et al. [Barosan 2006]. It is based on the calculation and extrapolation of the spectrum of the Legendre discretisation. 
The approximate solution of order $P$ in each element can be written as

$$
u^{P}=\sum_{n=0}^{P} a_{n} L_{n},
$$

where $L_{n}$ is the Legendre polynomial of degree $n$ and $a_{n}$ is the spectral coefficient (i.e. modal energy). The estimation of the discretisation error for the Legendre polynomial approximation is

$$
\epsilon_{P}^{N}=\left(\frac{a_{N}^{2}}{\frac{1}{2}(2 N+1)}+\int_{N+1}^{\infty} \frac{[a(n)]^{2}}{\frac{1}{2}(2 N+1)}\right)
$$

where function $a(n)$ is a least squares best fit of the last six points of the spectrum. To perform the least squares best fit an exponential decay, $a(n) \sim c e^{-\sigma n}$, is assumed. Note that this method estimates the discretisation error, but may be viewed as an estimator for the smoothness (i.e. determines the order polynomial) and hence as a "feature based adaptation" method.

\subsubsection{Discretisation Error Adaptation Algorithm}

The discretisation error based adaptation algorithm uses the estimate Eq. 4.8 that is based on the spectral coefficients. The complete algorithm is detailed in Algorithm 3 and is performed on each mesh element. In part A, the simulation is converged until steady state and the coefficients $a_{k}$, and $\epsilon_{P}^{N}$ are estimated.

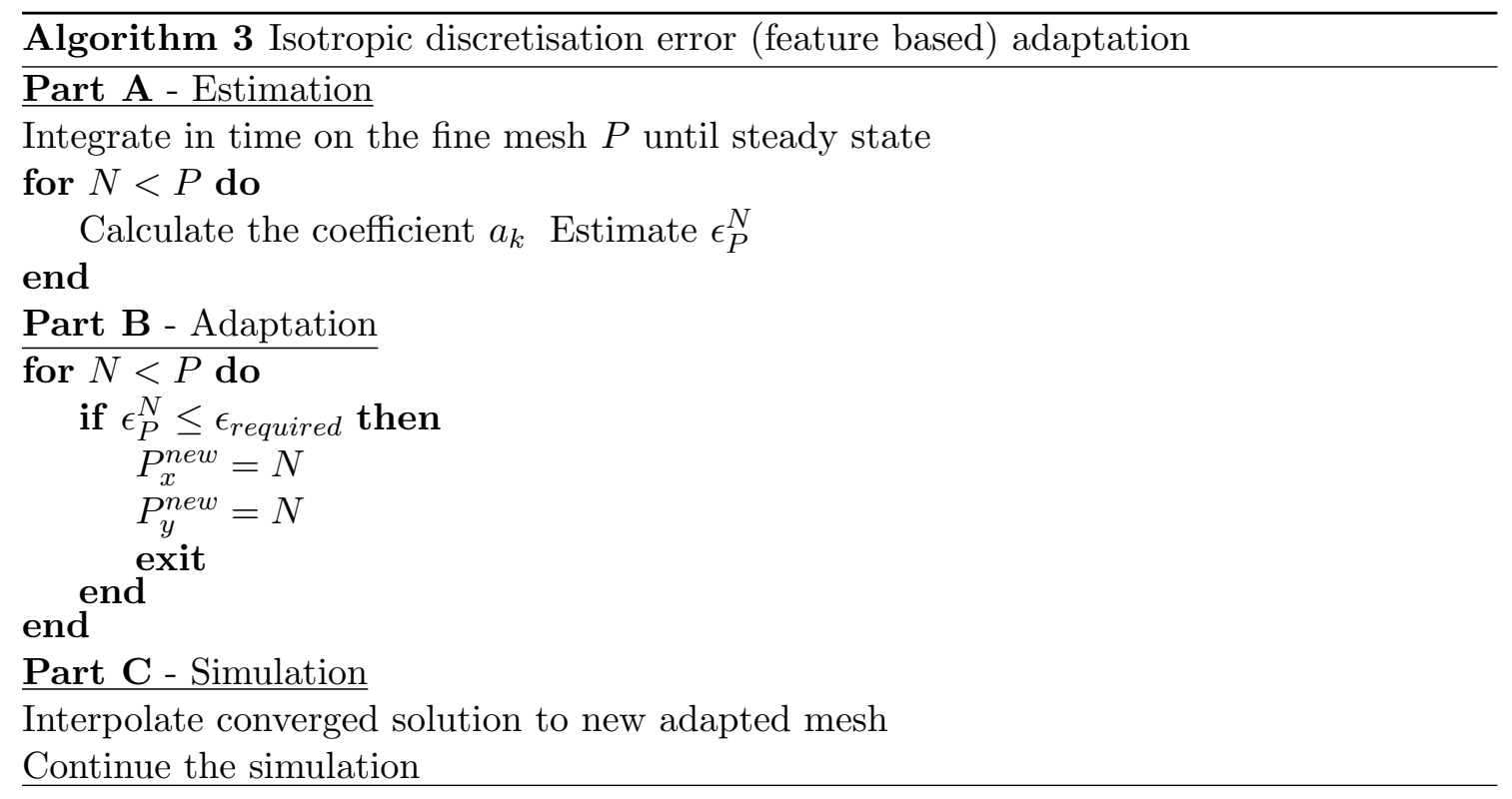

In part B, a new polynomial order is chosen such that the discretisation error estimation $\epsilon_{P}^{N}$ is below the desired threshold $\epsilon_{\text {required }}$. Having selected the polynomial that fulfills the threshold, the mesh is adapted by refining or coarsening the necessary element. In part $\mathrm{C}$, the solution is interpolated into the new adapted mesh and the simulation is restarted and continued until convergence. 


\subsection{Anisotropic Adaptation}

\subsubsection{Truncation / Isolated Truncation Error Adaptation Algorithm}

The anisotropic adaptation approach based on the truncation error is shown in Algorithm 4. The main difference to Algorithm 2 is that the estimates are decoupled and considered independently in each direction. For example, in the case of a two dimensional problem the polynomial order is fixed to the maximum in one direction and the estimates are determined for the other directions eg. $\tau_{P}^{1: N_{\max }}$. This is done in part $\mathrm{B}$ of the algorithm. Accordingly the required polynomial order is selected independently in each direction. If the error can not be obtained, extrapolation can be used to obtain the estimates for the missing values. This is done like in Algorithm 2 where a one-dimensional error extrapolation is used. To perform the extrapolation for a two dimensional problem, one coordinate direction is set to the maximum polynomial order while the values for the other directions will be used to extrapolate to higher orders (and vice versa).

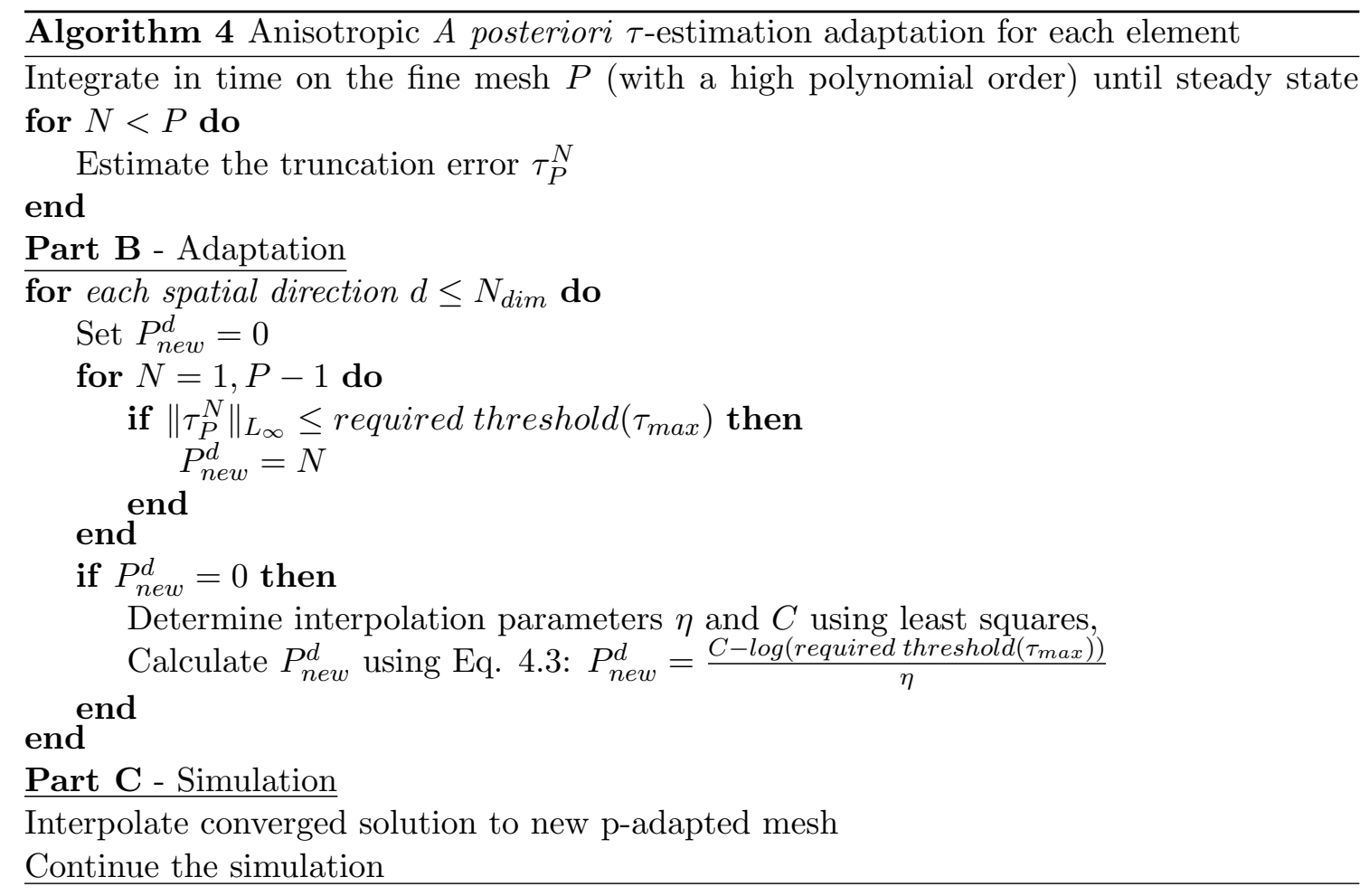

\subsubsection{Minimization of DoF}

In the previous section an anisotropic truncation error based adaptation algorithm, that makes use of the estimate Eq. 3.39 and error extrapolation, was introduced. This algorithm can be extended to minimize the DoF. A minimal DoF is advantageous to guarantee a small computational cost and a reduced memory usage.

The complete procedure that encompasses minimization of DoF is detailed in Algorithm 5. In part A, the simulation is converged until steady state and the truncation error 
is estimated for all coarser meshes $\left(N_{x}<P, N_{y}<P\right)$. In part $\mathrm{B}$, the new polynomial order is chosen. This part was modified such that the error threshold is satisfied, using the minimum local number of degrees of freedom (LDoF), for each mesh element.

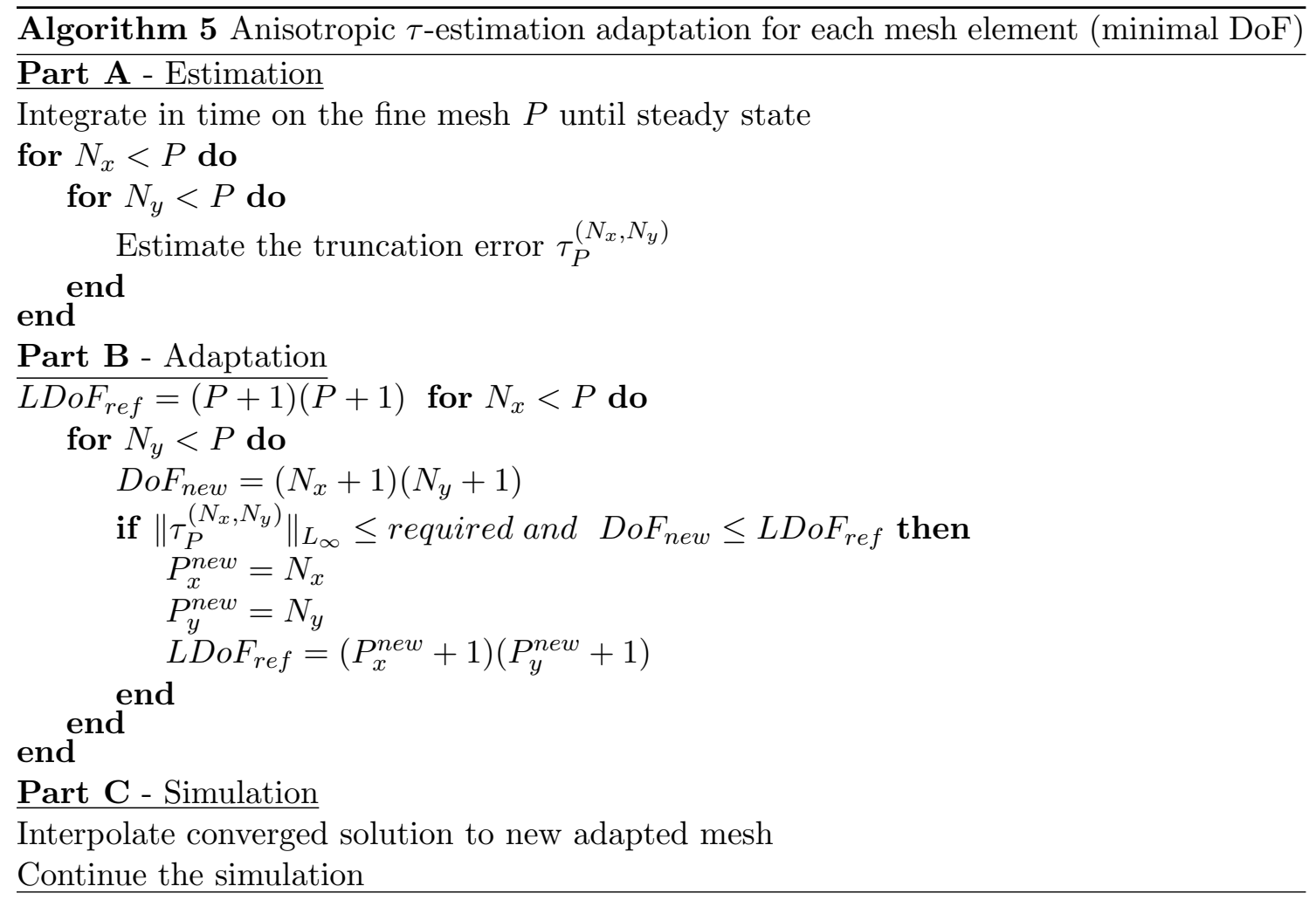

The adaptation algorithm for one mesh element is illustrated in Figure 4.5. Selecting $P=8$ as reference, the truncation error $\tau_{P}^{\left(N_{x}, N_{y}\right)}$ is calculated (presented in contour colors) using the lower polynomials $\left(N_{x}=1: 7 N_{y}=1: 7\right)$ in each direction. Figure 4.5 (left) shows the truncation error estimates. In this case, a required truncation error required $=10^{-2}$ cannot be achieved by polynomial combinations of low order (eg. red, yellow and light green areas). However, the required error is below the required threshold in the upper right (blue) area.

After these valid combinations are identified, the optimal one is selected as the combination with the lowest number of LDoF. Figure 4.3 (right) shows the number of LDoF for each of the combinations. It can be seen that $N_{x}=4$ and $N_{y}=5$ guarantees the required threshold error, while minimising the local number of degrees of freedom to $\mathrm{LDoF}=30$.

In part $\mathrm{C}$, the solution is interpolated into the new mesh and the simulation restarted. The algorithm can be used for the isolated truncation error by using Eq. 3.47 instead of Eq. 3.39 .

\subsubsection{Error Extrapolation for minimal DoF}

Using anisotropic refinement together with a minimisation of the DoF, the error extrapolation is changing due to the two dimensional character of the error estimation. Figure 

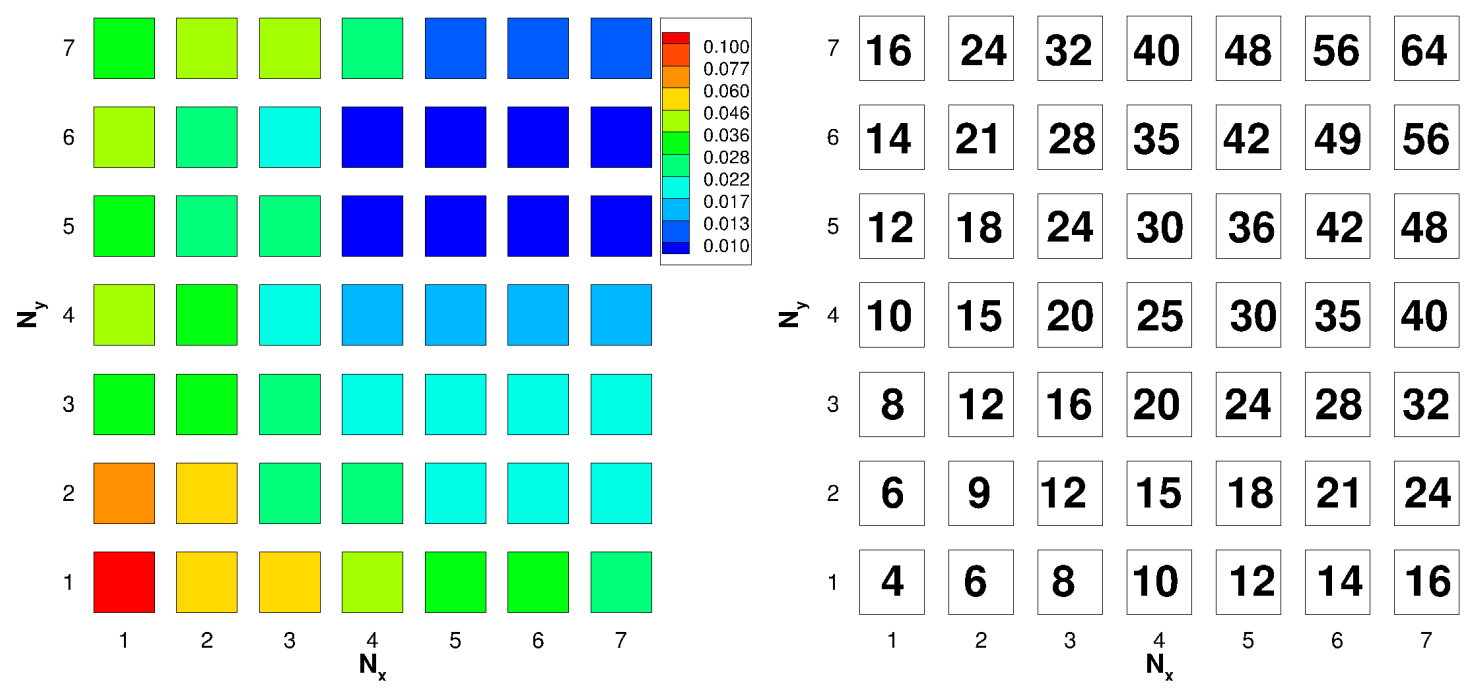

Figure 4.3: Truncation error estimation for different combinations of polynomial orders (left) and corresponding LDoF (right).

4.3 showed the estimates for each of the combinations of $\tau_{P}^{\left(N_{x}, N_{y}\right)}$ for $N_{x}=N_{y}=1, \ldots, 7$. To obtain an estimate outside of this area the values have to be extrapolated in a two dimensional manner. To obtain these results, a simple least square fitting of the form $z=a x+b$ has been presented in section 4.1.2 to obtain the truncation error estimates for the higher polynomial orders.

For this anisotropic two dimensional extrapolation, one dimension has to be added, see [Lancaster 1986, Lawson 1995] for more details on least square problems. The least square regression plane is now of the form $z=a x+b y+c$. Figure 4.4 illustrates the extrapolation in three dimensions. It can be seen that for a combination of high polynomial orders (eg. $N_{x}=10 N_{y}=9$ ), the error is lower than for a combination of lower orders (eg. $N_{x}=2 N_{y}=2$ ). It should be noted that this plane-fitting is only possible due to the exponential scaling of the truncation error and under the assumption of the asymptotic range. Furthermore, this plane can be tilted or rotated for elements with predominant features, for example a faster decay of the error in $N_{x}$ direction.

The optimal values a,b and c for least square regression plane are the values that minimize the equation

$$
G(a, b, c)=\sum\left(z_{i} a x_{i}-b y_{i}-c\right)^{2}
$$

and can be determined by solving the system $\partial G / \partial a=0, \partial G / \partial b=0, \partial G \partial c=0$. Due to the linearity, these values can be determined solving the following system

$$
\left[\begin{array}{ccc}
\sum N_{i x}^{2} & \sum N_{i x} N_{i y} & \sum N_{i x} \\
\sum N_{i x} N_{i y} & \sum N_{i y}^{2} & \sum N_{i y} \\
\sum N_{i x} & \sum N_{i y} & N_{x} N_{y}
\end{array}\right]\left[\begin{array}{c}
a \\
b \\
c
\end{array}\right]=\left[\begin{array}{c}
\sum N_{i x} \tau \\
\sum N_{i y} \tau \\
\sum \tau
\end{array}\right]
$$




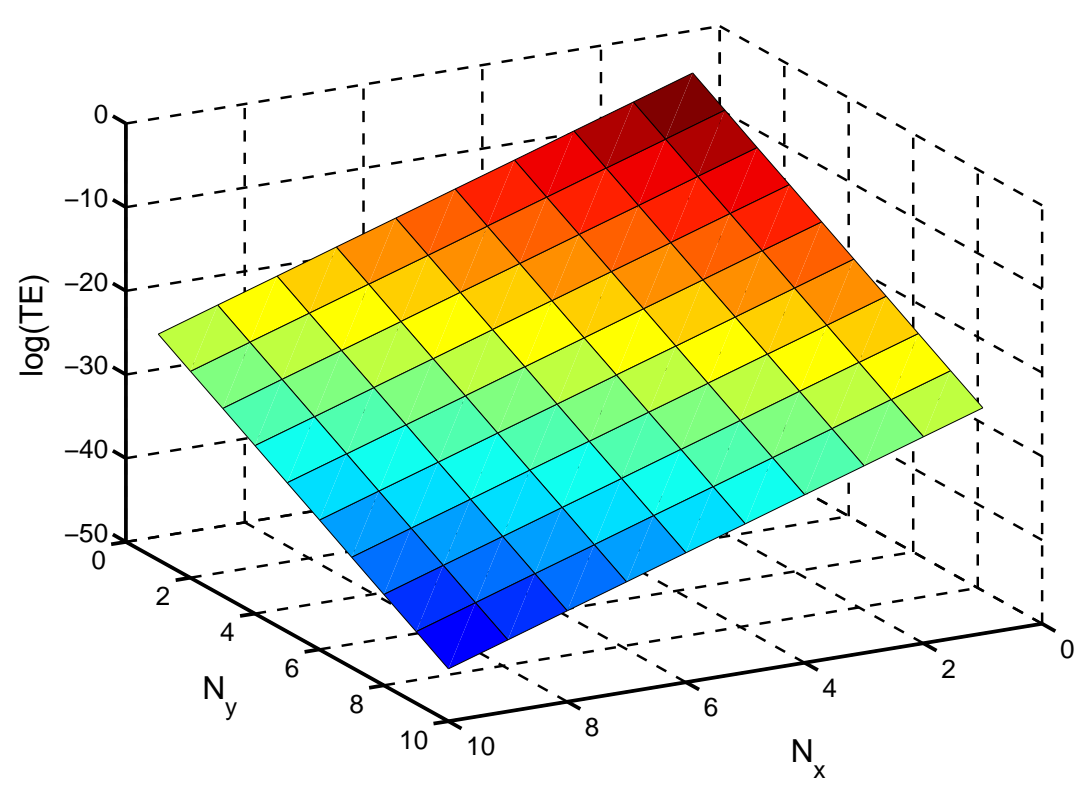

Figure 4.4: Illustration of truncation error plane fitting used for 2D extrapolation, Polynomial orders $N_{x}$ and $N_{y}$ vs truncation error value $\log (T E)$ (different color level).

After calculating the extrapolated error values, the scatter plot can be extended, see Figure 4.5

The properties of the extrapolation can be shown using a simulation with polynomial order $P=9$ on the fine mesh, without extrapolation and comparing them with a simulation using $P=7$ with extrapolation. The two dimensional estimates are shown in Figure 4.6. On the left side the estimates $\tau_{9}^{N_{x}, N_{y}}$ for the fine solution with $P=9$ are shown. On the right side the same simulation is shown using a fine solution with $P=7$ and extrapolating these values until $P=8$. It can be seen that these values generally agree well to with each other while some small differences remain.

In the case that the extrapolation does not work well, it might be necessary to exclude the low order estimates from the extrapolation procedure. It is possible that these values are not in the asymptotic convergence range and an exponential scaling can not be guaranteed. 

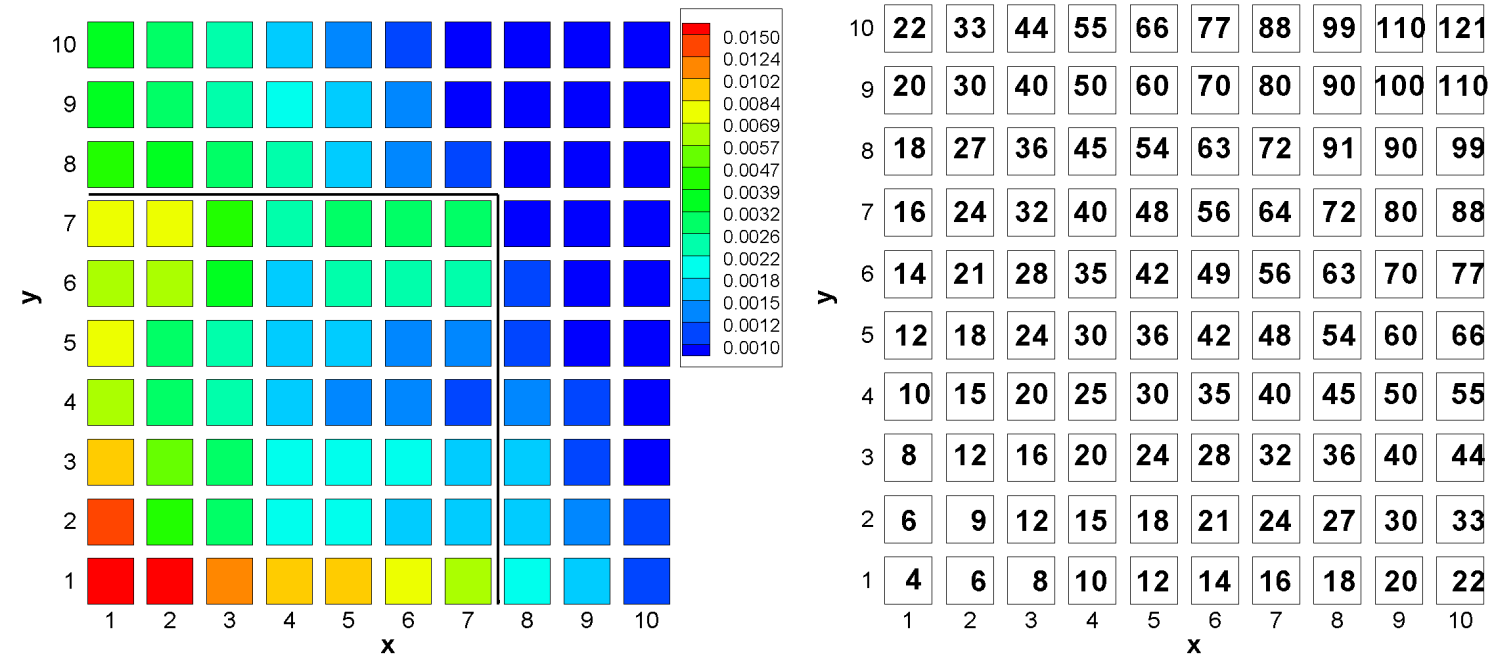

Figure 4.5: Truncation error estimation for different combinations of polynomial orders (left) with extrapolated values and corresponding LDoF (right).
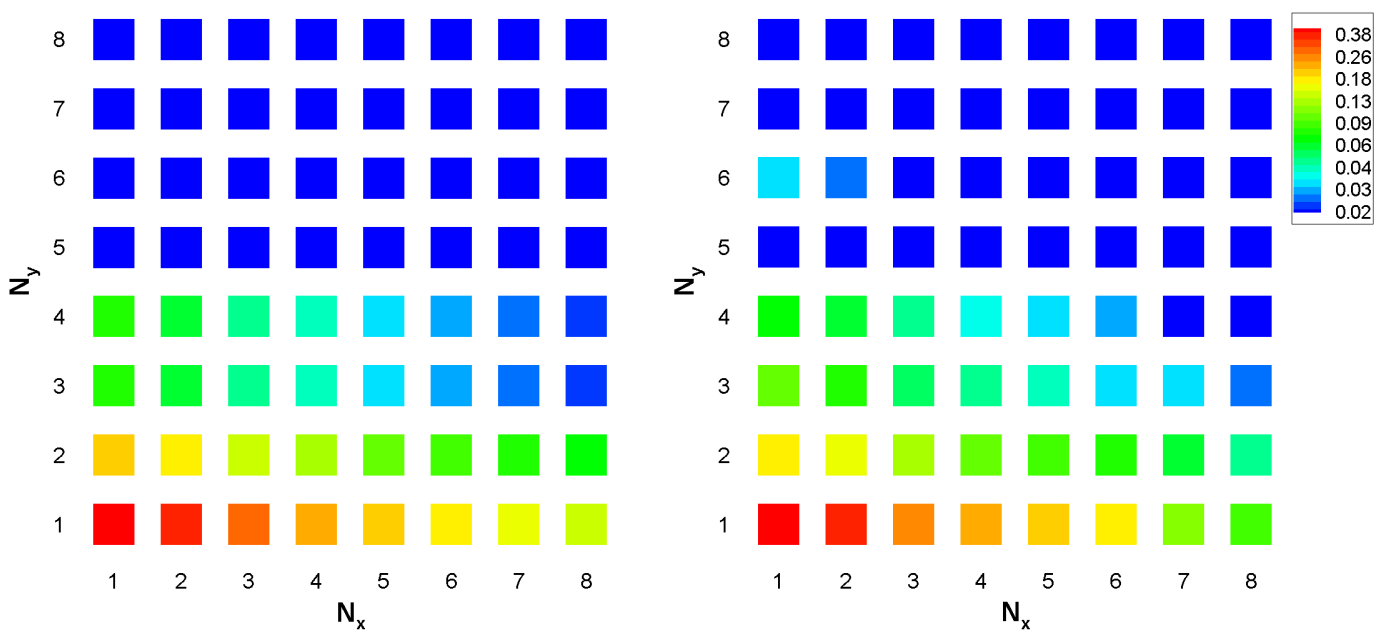

Figure 4.6: Truncation error with without extrapolation (left) and extrapolated values (right). 


\subsection{Quasi-a priori and quasi- a priori corrected $\tau$-estimation}

The adaptation process for the non-converged quasi- $a$ priori $\tau$-estimation, described in Algorithm 4, is similar to the a posteriori $\tau$-estimation. The main difference with respect to the previous algorithm is that the solution used for the estimation is only partially converged in time. In this work, the infinite norm of the residual (tolerance) is chosen as the criterion to stop the computation. According to Eq. 3.43, the difference between the estimated and the exact truncation error is caused by two different sources. The first term is proportional to the discretization error in a finer mesh $\epsilon^{P}$, which is negligible due to the spectral convergence of the method if $P>N$. The second term is proportional to the square of the iteration error (if the correction is applied) or to the iteration error (if no correction is applied). However, for smooth solutions, the iteration error is proportional to the residual, Eq. 3.44, which is the typical parameter monitored in the convergence process. With these considerations in mind and once the desired maximum error threshold is defined $\left(\tau_{\max }\right)$, the following criteria can be used:

1. Quasi- $a$ priori: If no correction term is considered (to reduce the computational cost), the maximum value of the residual is chosen such that the solution converges until tolerance $<\tau_{\max } / F$, namely, the residual is an order of magnitude lower than the desired maximum threshold. A typical value for $F$ is 10 . This guarantees that Eq. 3.43 holds when retaining first order terms and then Eq. 3.39 provides an accurate estimation of the truncation error.

2. Quasi- a priori corrected : If the correction term is applied, Eq. 3.43 applies with $\mathcal{O}\left(\epsilon_{i t}^{P}\right)^{2}$. Therefore, the value of the residual can be relaxed to tolerance $<\left(\tau_{\max } / F\right)^{1 / 2}$.

Once the tolerance is reached, the truncation error is estimated and the appropriate polynomial order is chosen in part B of the algorithm, as detailed in the previous section. In the final step the non-converged solution is interpolated onto the p-adapted mesh and the simulation is continued.

It should be noted, that when anisotropic p-adaptation is considered, the algorithms are performed for each cell in the computational domain. The principal directions in the computational domain correspond to the physical $x$ and $y$ - directions only when the mesh is aligned with the Cartesian coordinate system.

\subsubsection{Memory Requirements}

To estimate the truncation error estimation or the isolated truncation error on the fine mesh $P$, the memory requirements will at least double in comparison to no estimation and considering the non corrected approach. In [Hindenlang 2012], the generally low memory requirements of the DGSEM method are described (for the Navier-Stokes equations discretized with 1 million $\mathrm{DoF} \approx 0.5 \mathrm{~Gb}$ ). 
The computation of the correction term requires the additional storage of the Jacobian matrix for the fine and the coarse mesh. The memory requirements to store a square matrix of dimension DoF $=10^{6}$ is $\approx 8000 \mathrm{~Gb}$. Nevertheless, due to the compactness of the scheme the Jacobian can be stored in sparse form and the memory requirements will decrease drastically. According to [Rubio 2015], each DoF of the Jacobian only includes information about the element and its neighbours. Consequently, for a two dimensional problem it follows,

$$
\frac{\text { Jacobian non zeros }}{\text { Jacobian total }} \approx \frac{5 \times \operatorname{DoF} \times(N+1)^{2}}{\operatorname{DoF}^{2}}=\frac{5(N+1)^{2}}{\mathrm{~N}_{e l}(N+1)^{2}}=\frac{5}{\mathrm{~N}_{e l}} .
$$

An estimated memory requirement for a sparse Jacobian of a $2 \mathrm{D}$ problem with $\mathrm{DoF}=10^{6}$ and polynomial order $N=4$ is $\approx 1 \mathrm{~Gb}$.

\subsubsection{Theoretical Computational Cost}

In this subsection the computational cost is analysed and an estimation in terms of performed operations are given following the theory of [Rubio 2015, Hindenlang 2012]. A temporal cost analysis for a boundary layer test case will be presented later in section 5.5.

In equation 3.39 the error estimates for the truncation error are obtained based on a non converged quasi- $a$ priori estimation. The same method is used to obtain the estimates for the isolated truncation error in 3.47.

For a non-linear operator the following steps are necessary:

1. To obtain the terms $\mathcal{R}^{N}\left(\tilde{u}^{P}\right)$ or $\hat{\mathcal{R}}^{N}\left(\tilde{u}^{P}\right)$ :

$1.1 \tilde{u}^{P}$. Obtain non time-converged solution on a fine mesh

$1.2 \mathcal{R}^{N}\left(\tilde{u}^{P}\right)$ or $\hat{\mathcal{R}}^{N}\left(\tilde{u}^{P}\right)$. Evaluate the discrete partial differential operator with $\tilde{u}^{P}$ on the on the coarse mesh.

2. To obtain the terms $\bar{I}_{P}^{N} \mathcal{R}^{P}\left(\tilde{u}^{P}\right)$, the correction term for quasi-a priori $\tau$-estimation:

$2.1 \epsilon_{i t}^{P} \approx\left(\left.\frac{\partial \mathcal{R}^{P}}{\partial u^{P}}\right|_{\tilde{u}^{P}}\right)^{-1} \mathcal{R}^{P}\left(\tilde{u}^{P}\right)$. Approximate iteration error on the fine mesh, see the definition of the transfer operators (3.42) and (3.48).

$\left.2.2 \frac{\partial \mathcal{R}^{N}}{\partial u^{N}}\right|_{\tilde{u}^{P}} I_{P}^{N}$ or $\left.\frac{\partial \hat{\mathcal{R}}^{N}}{\partial u^{N}}\right|_{\tilde{u}^{P}} I_{P}^{N}$. Transfer of the residual from the fine to the coarse mesh.

For an explicit Euler time-iterative method step 1.1 requires approximately $\mathrm{N}_{i t} \times \operatorname{DoF} \times P$ operations, where $\mathrm{N}_{i t}$ is the number of iterations. Step 1.2 needs less than $2 \times \operatorname{DoF} \times P$ operations and is thereby negligible in comparison to 1.1 .

To calculate the estimate of the iteration error 2.1, a linear system has to be solved. This step highly depends on the efficiency of the algorithm that solves the linear system. As an example, the inversion of the Jacobian on a fine mesh by Gauss-Jordan algorithm is less than $(D o F)^{3}$ operations. Finally, for the transfer of the residual to the coarse mesh 


\begin{tabular}{|l||l|l|l|}
\hline & a posteriori & quasi- a priori corrected & quasi- a priori \\
\hline \hline Residual & machine error & ${\text { (tolerance })^{1 / 2}}^{\prime 2}$ & tolerance \\
\hline Correction term & No & Yes & No \\
\hline Operation count & $\mathrm{N}_{i t 1} \times$ DoF $\times P$ & $\mathrm{~N}_{i t 2} \times \operatorname{DoF} \times P+(\mathrm{DoF})^{3}$ & $\mathrm{~N}_{i t 3} \times \operatorname{DoF} \times P$ \\
\hline Memory requirements & Low & High & Low \\
\hline
\end{tabular}

Table 4.2: Computational features of the estimation procedures.

2.2, another matrix vector multiplication is necessary, which results in approximately $\left(D o F_{\text {coarse }}\right)^{2}$ operations.

For multiple evaluations of the correction term, in case of the non time-converged solution, the step 2.1 remains the same. That means that the step of solving the linear system, which is the most expensive operation, only has to be performed once and only the projections to the coarse meshes $N$ are carried out several times.

A full overview of the three estimation procedures can be seen in Table 4.2. Further information can be found in [Rubio 2015]. 


\section{Numerical Results}

\section{Contents}

5.1 Manufactured Solution Test Case . . . . . . . . . . . . . . . . . . 60

5.1.1 Estimated Error vs. Exact Error . . . . . . . . . . . . . . . . 61

$5.1 .2 \quad$ A posteriori and quasi-a priori Estimates . . . . . . . . . . 62

5.1 .3 Adaptation . . . . . . . . . . . . . . . 63

5.2 Inviscid NACA0012 Airfoil . . . . . . . . . . . . . . . . . . 67

5.3 Boundary Layer Test Case . . . . . . . . . . . . . . . . . . . . . 72

5.3 .1 Adaptation . . . . . . . . . . . . . . . . . . . . . 74

5.3.2 Adaptation Strategy Comparison . . . . . . . . . . . . . . 76

5.3.3 Reynolds Number Variations _. . . . . . . . . . . . . . 78

5.4 Cylinder Test Case . . . . . . . . . . . . . . . . . . . 87

5.5 Computational Cost . . . . . . . . . . . . . . . . . 92

In order to check the accuracy and efficiency of the described methodology, different test cases are presented.

First, the truncation error is estimated and the anisotropic adaptation algorithm is applied to a manufactured solution problem, where an exact solution is available. The error estimation procedure and the efficiency of the different strategies for adaptation ( $a$ posteriori, quasi-a priori and quasi- $a$ priori corrected approaches) are analyzed.

Second, an inviscid NACA problem is used to demonstrate the properties of the error estimation and adaptation for test cases with a higher number of degrees of freedom and to show the potential of the anisotropic p-adaptation and adaptation based on the different sensors.

Third, a boundary layer problem is presented to show the properties of the adaptation process for the Navier Stokes equations and when singularities are present.

In all test cases and unless otherwise specified, the desired threshold truncation error is chosen to be $\tau_{\max }=10^{-5}$. A solution is assumed to be converged when the residual of the solution (tolerance) is below $10^{-10}$. For non-converged solutions, a value of tolerance $=$ $10^{-6}$ is chosen, if no correction term is applied, and $10^{-3}$ for the cases with correction. 


\subsection{Manufactured Solution Test Case}

The manufactured solution technique can be used to determine the ability of an error estimation method, see Roy et al. [Roy 2002]. This technique requires forcing terms to drive the differential partial equations to a predetermined solution. These source terms are incorporated in the code, then the modified governing equations (including the source terms) are discretized and solved numerically and compared to the exact solution.

Based on Roy [Roy 2002], the six steps to implement the method of manufactured solutions are: 1 . Choose the form of the governing equations, 2. Choose the form of the manufactured solution, 3. Apply the governing equations to the manufactured solution to generate analytical source terms, 4. Discretize the equations using analytical boundary conditions and source terms from the manufactured solution, 5. Evaluate the truncation error in the numerical solution and 6. Determine whether the observed order of accuracy matches the formal order of accuracy.

In our case, the previous steps are applied to the 2D inviscid Euler equations

$$
\mathbf{Q}_{t}+\mathbf{F}_{x}^{a}+\mathbf{G}_{y}^{a}=\varphi(x, y) .
$$

where $\mathbf{Q}$ is $(\rho, \rho u, \rho v, \rho e)^{T}$ and $\rho, u, v, e$ denote the density, velocity components and energy. The inviscid flux vectors $\mathbf{F}^{a}$ and $\mathbf{G}^{a}$ are

$$
\mathbf{F}^{a}=\left[\begin{array}{c}
\rho u \\
p+\rho u^{2} \\
\rho u v \\
u(\rho e+p)
\end{array}\right], \mathbf{G}^{a}=\left[\begin{array}{c}
\rho v \\
\rho u v \\
p+\rho^{2} \\
v(\rho e+p)
\end{array}\right],
$$

where the pressure $p$ is assumed to follow an ideal gas equation. For the imposed source term the horizontal and vertical velocities are set constant $(u=v=1)$, while the pressure and density distribution are chosen to follow an exponential distribution:

$$
\begin{array}{r}
\rho(x, y)=e^{-5\left(4(x-0.5)^{2}+(y-0.5)^{2}\right),} \\
u(x, y)=1, \\
v(x, y)=1, \\
p(x, y)=e^{-5\left(4(x-0.5)^{2}+(y-0.5)^{2}\right)} .
\end{array}
$$

This exponential function is steep with large gradients in the $x$-direction while it is relatively flat in the $y$-direction, see Figure 5.1. This shape is used to test the error estimation as it is expected that a denser refinement in $x$-direction is needed while a less stringent refinement is required in the $y$-direction. This set of manufactured solutions is infinitely differentiable and no derivatives vanish. Furthermore the solutions are sufficiently smooth to guarantee a fast convergence towards the asymptotic rate. Clearly, this set of 


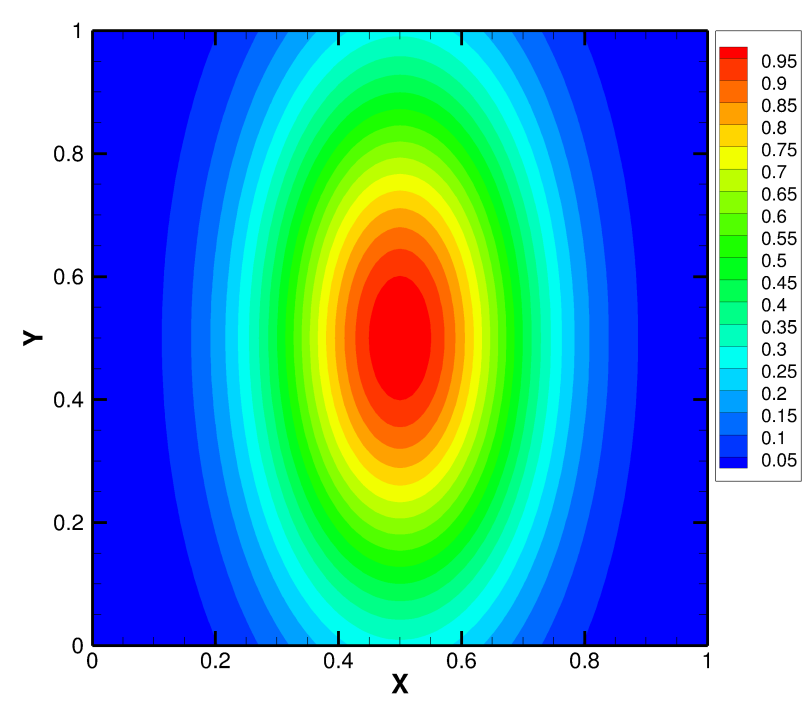

Figure 5.1: The function $\rho(x, y)=p(x, y)=e^{-5\left(4(x-0.5)^{2}+(y-0.5)^{2}\right)}$ is used for the source term of the manufactured solution.

equations is not satisfying the original Euler equations. Additional source terms have to be added according to the Manufactured solutions. One can use symbolic differentiation or automatic code generation, see [Roache 1998]. As an example, inserting 5.3 into the Euler equations, the following source terms can be obtained for the continuity equation:

$$
\begin{aligned}
S_{\rho}=-(40 x-20) / \exp (20 & \left.(x-1 / 2)^{2}+5(y-1 / 2)^{2}\right) \\
& -(10 y-5) / e^{20(x-1 / 2)^{2}+5(y-1 / 2)^{2}}
\end{aligned}
$$

$$
\begin{aligned}
S_{\rho u}=-(40 x-20) / \exp ( & \left.20(x-1 / 2)^{2}+5(y-1 / 2)^{2}\right) \\
-(40 x-20) / e^{20(x-1 / 2)^{2}+5(y-1 / 2)^{2}} & -(10 y-5) / e^{20(x-1 / 2)^{2}+5(y-1 / 2)^{2}}
\end{aligned}
$$

The test case is performed with a Mach number of $\mathrm{M}=0.8$.

\subsubsection{Estimated Error vs. Exact Error}

First, the estimation of the truncation error is calculated using the a-posteriori approach. As already mentioned, the manufactured solution test case is converged when the residual infinite norm falls below the prescribed tolerance: $\left\|\mathcal{R}^{N}\left(u^{N}\right)\right\|_{L_{\infty}}<$ tolerance $=10^{-10}$. The calculation is obtained with a polynomial order of $P_{x}=P_{y}=8$ on each element. 
Based on this calculation, and using Eq. 3.41 the truncation error is estimated for all combinations of the polynomial orders $N_{x}=1, \ldots, 7$ and $N_{y}=1, \ldots, 7$. Note that the DGSEM method enables different polynomial orders to be used in each spatial direction.

In Figure 5.2, the results obtained for the truncation estimates are depicted for a 4 x 4 (top) and $10 \times 10$ (bottom) grid. The left side of the picture shows the estimated map $\tau_{8}^{7}$ and the right side shows the exact truncation error $\tau_{\text {exact }}^{7}$ (i.e. the exact solution interpolated using a uniform polynomial order 7). It can be seen that the estimate agrees very well with the exact solution for both meshes and that the maximum relative error: $\left.\left\|\tau_{8}^{7}-\tau_{\text {exact }}^{7}||_{L_{\infty}} /\right\| \tau_{\text {exact }}^{7}\right|_{L_{\infty}}$, is below 0.106 for the $4 \times 4$ mesh and below $5.399 \cdot 10^{-2}$ for the 10x10 mesh.

In addition, two individual elements for each grid, A and B are selected, to explore how the polynomial order influences the error. Figure 5.3 shows the errors for Element A of the $4 \times 4$ mesh for different polynomial orders. As expected, the error decreases for higher orders. Furthermore, the distribution is not symmetric along the diagonal (i.e. $N_{x}=N_{y}$ ). This could have been expected since the truncation error is stretched in the $x$-direction and thus a more stringent refinement is needed in this direction when compared to the $y$-direction.

The same tendency can be observed for the element B of the 10x10 mesh, Figure 5.4. In this case, the error decreases significantly faster, with a difference of 8 orders of magnitude, when using polynomial orders $N_{x}, N_{y}$ ranging from 1 to 7 .

Finally, the truncation error dependence with the polynomial order is shown in Figure 5.5. The polynomial order in the $y$-direction is fixed to $N_{y}=7$ and only the error related to $N_{x}$ is considered. In both cases the estimates are in the asymptotic range, but due to a higher density of interpolation points, the error for the 10x10 mesh (right side) is lower for the same polynomial when compared to the $4 \mathrm{x} 4$ mesh (left side). The estimates are validated using the exact solution, Eq. 5.3, red line in Figure 5.5, which states that the estimates agree very well with the exact errors.

As described in the previous section, for a set truncation error threshold, these contours are used to estimated the polynomial order needed to obtain the desired accuracy.

\subsubsection{A posteriori and quasi-a priori Estimates}

In this section, the previously computed a posteriori approach is compared to the quasia priori one. Figure 5.6 shows the truncation error on one particular element for the manufactured solution test case $(10 \times 10$ mesh). For this plot, the truncation error was estimated in the a posteriori approach after the simulation was converged until a tolerance of $10^{-10}$. It can be seen that the truncation error for this simulation is of the same order of magnitude and that it decreases asymptotically with high polynomial orders. The quasia priori approach was converged until $10^{-3}$, while the truncation error was estimated once with and another time without applying the correction. Considering the estimations 


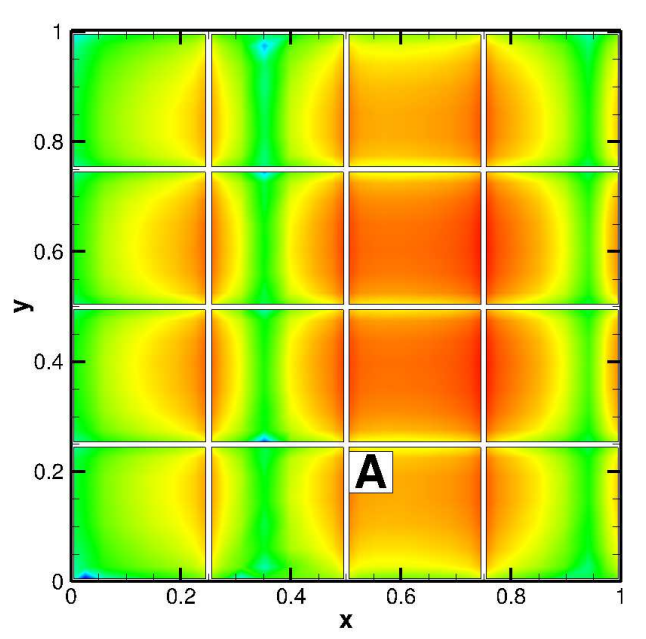

(a) $\tau_{8}^{7}$ on $4 \times 4$ mesh

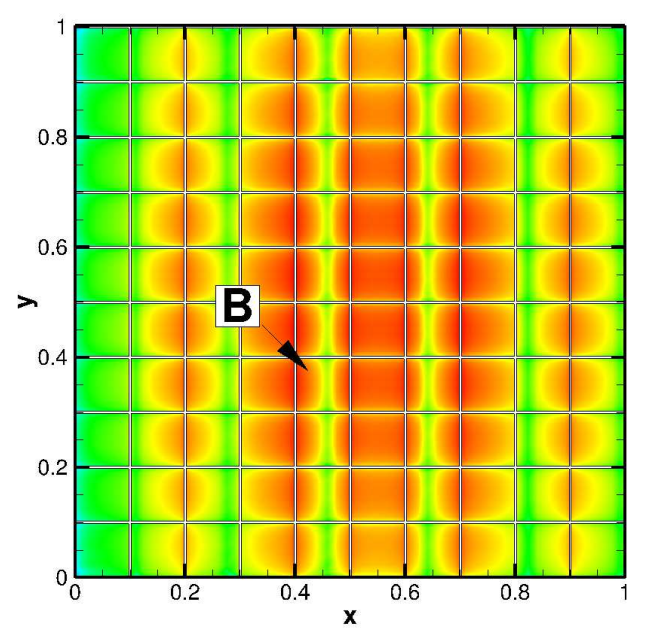

(c) $\tau_{8}^{7}$ on $10 \times 10$ mesh

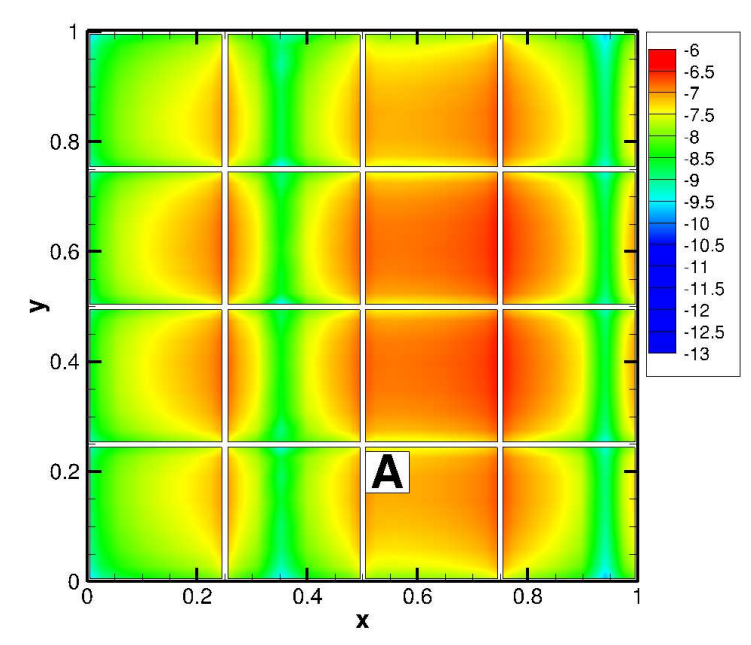

(b) $\tau_{\text {exact }}^{7}$ on $4 \mathrm{x} 4$ mesh

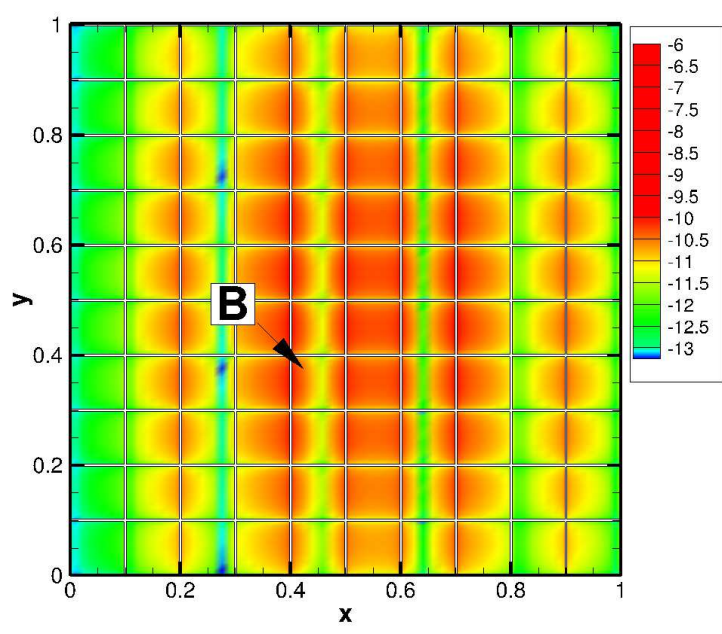

(d) $\tau_{\text {exact }}^{7}$ on $10 \times 10$ mesh

Figure 5.2: The truncation error estimation $\tau_{8}^{7}$ (left) and the exact truncation error $\tau_{\text {exact }}^{7}$ (right) on a $4 \times 4$ mesh (top) and 10x10 mesh (bottom) for polynomial order $N_{x}=N_{y}=7$. Colored contours show logarithmic values for the errors.

without the correction term, the error is stagnating around $10^{-3}$ while the estimations with the correction term flattens out around $10^{-6}$ to $10^{-7}$. Indeed, applying the correction term will cancel out the first order iteration error highlighted in Eq. 3.43.

\subsubsection{Adaptation}

Once the truncation error has been obtained, the a posteriori Algorithm 4, described in chapter 4 is applied, to adapt the mesh for different values of the truncation error threshold $\tau_{\text {max }}$. The required polynomial order in the $x$ and $y$ directions is obtained through interpolation (optimal scaling) or extrapolation (suboptimal scaling) of the truncation error 

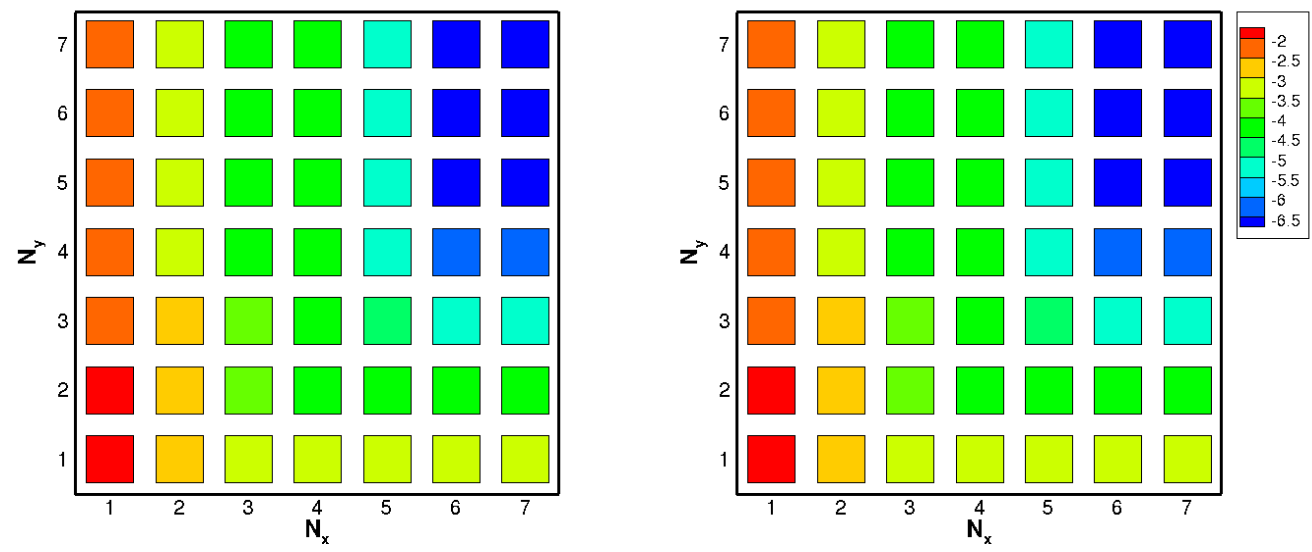

Figure 5.3: Scatter plot (logarithmic scale) for the truncation error $\tau_{8}^{N}$ for varying polynomial orders $N_{x}, N_{y}$ in the $4 \times 4$ grid and the manufactured solution case, only showing element A of Figure 5.2: left shows the estimated error and right shows the exact error.
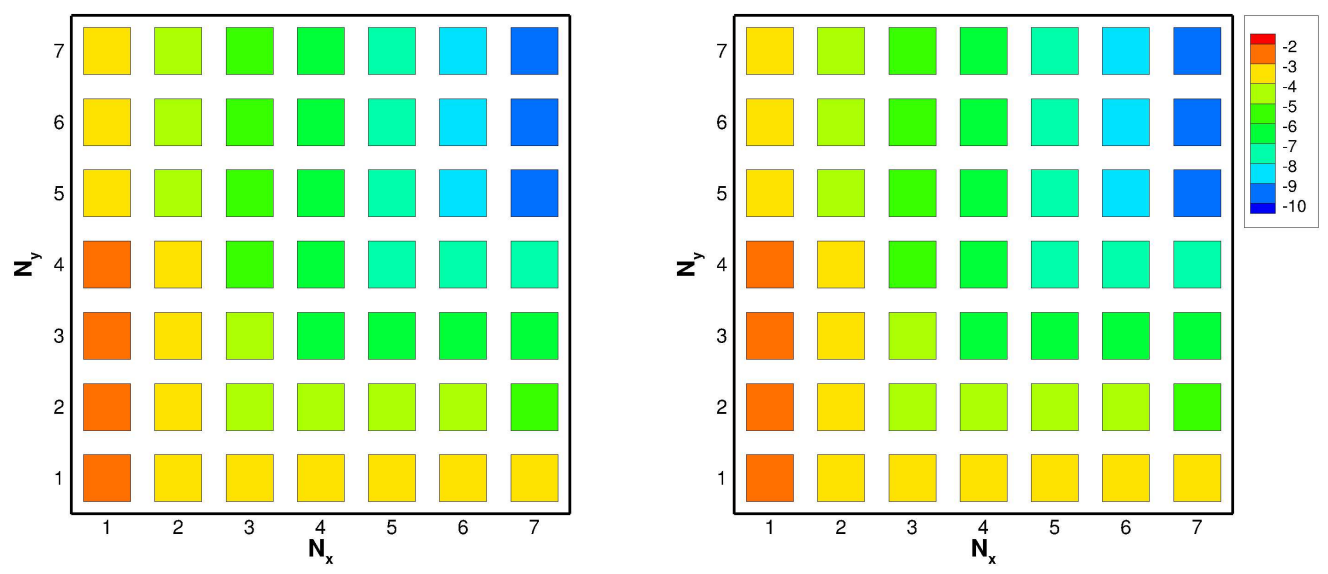

Figure 5.4: Scatter plot (logarithmic scale) for the truncation error $\tau_{8}^{N}$ for varying polynomial orders $N_{x}, N_{y}$ in the $10 \times 10$ grid and the manufactured solution case, only showing element B of Figure 5.2: left shows the estimated error and right shows the exact error.

estimates curves computed at each element (see the example in Figure 5.3).

Figure 5.7 shows the p-adapted meshes based on different threshold levels for the required truncation error. After the adaptation, the error is checked with the exact truncation error. The final error from the adapted mesh always achieves the required order of accuracy. It should be noted that the p-adapted mesh is finer in the $x$-direction than in the $y$-direction, which shows the potential of the method for anisotropic refinements. Note that the figure only shows interior element nodes. In the DGSEM formulation, these correspond to Legendre-Gauss nodes. Hence for a polynomial of order $P, P+1$ nodes per direction that do not include the edges of the element are shown. 

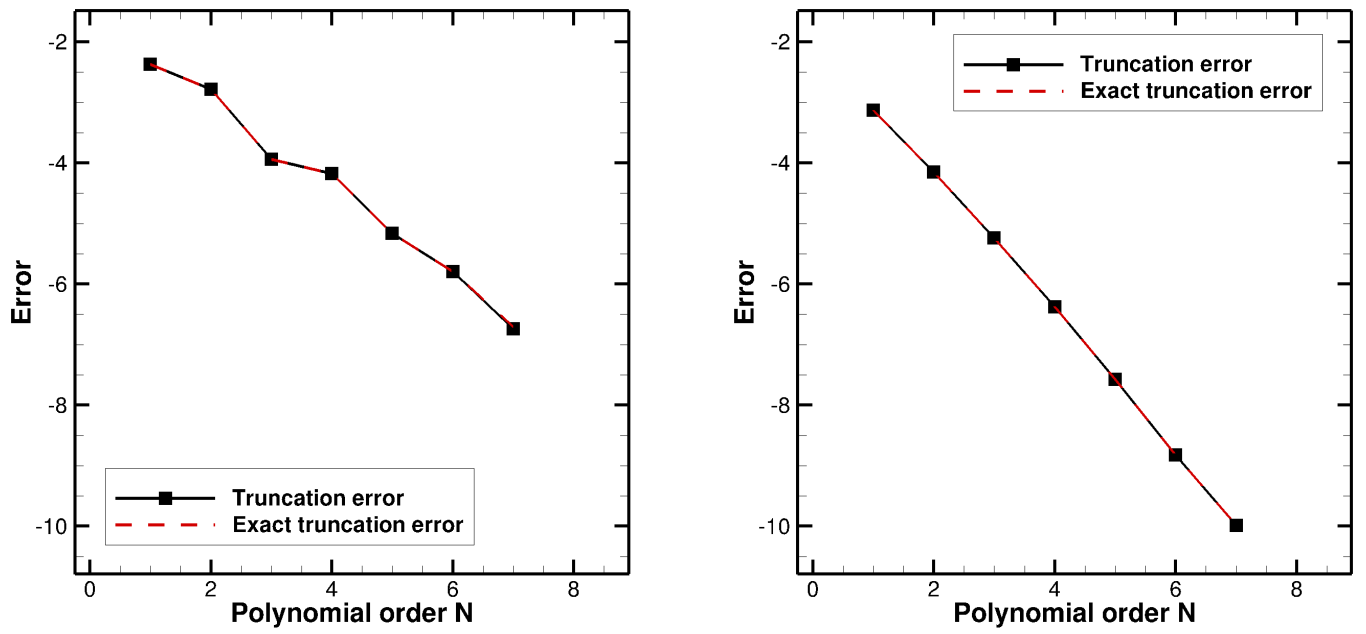

Figure 5.5: Logarithm of the estimated and exact truncation errors as a function of the polynomial order $N$ for $N=1, \ldots, 7$. The estimation is performed with a fine polynomial order $P=8$. Results are shown for the highlighted element A of the $4 \mathrm{x} 4$ mesh (left) and element B of the 10x10 mesh, see Figure 5.2.

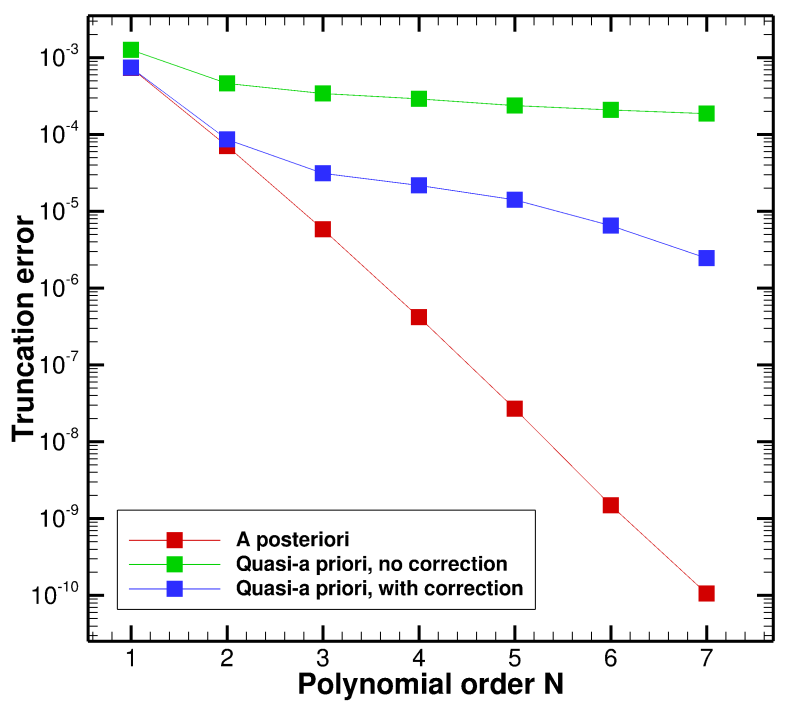

Figure 5.6: Truncation error estimation $\tau_{8}^{N}$ for $N=1, \ldots, 7$ : a posteriori, quasi- $a$ priori without correction and quasi-a priori with correction for the manufactured solution test case (10x10 mesh). 


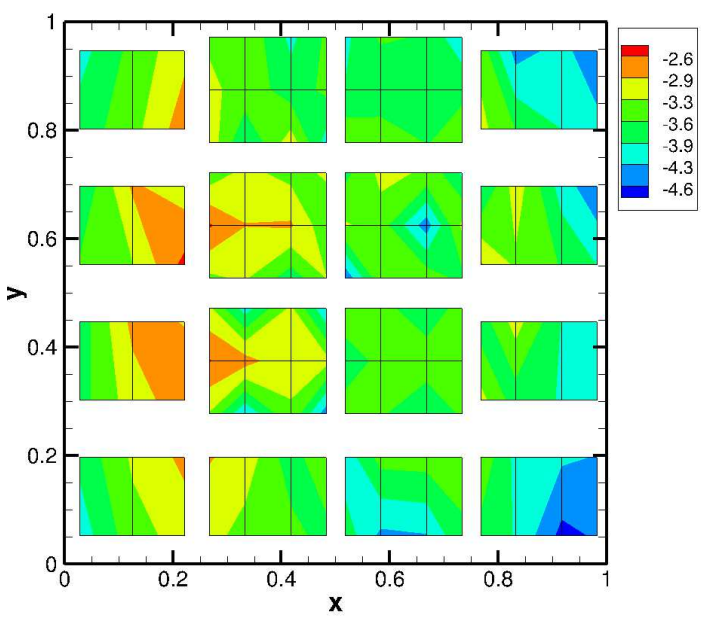

(a) $\tau_{\max }=10^{-3}$

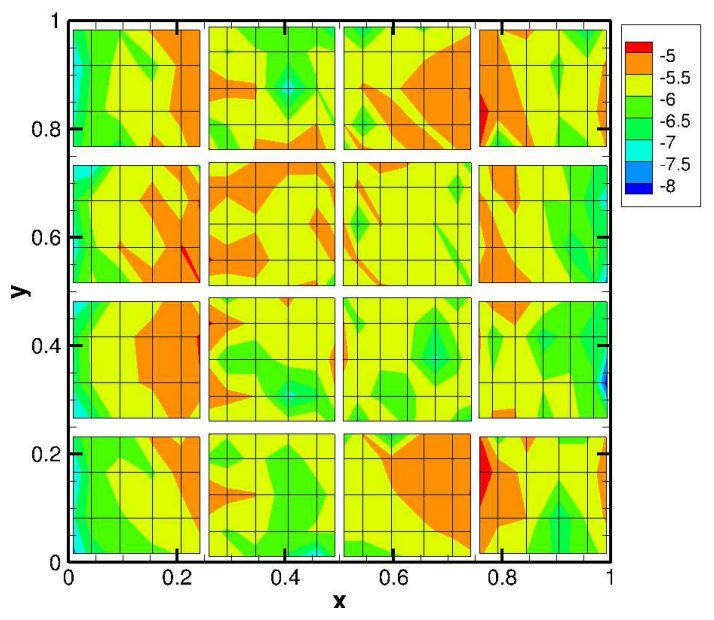

(c) $\tau_{\max }=10^{-5}$

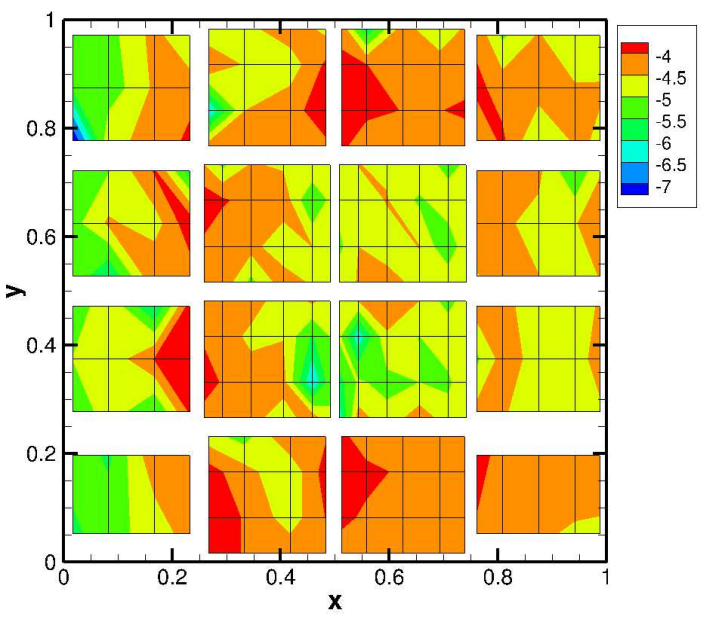

(b) $\tau_{\max }=10^{-4}$

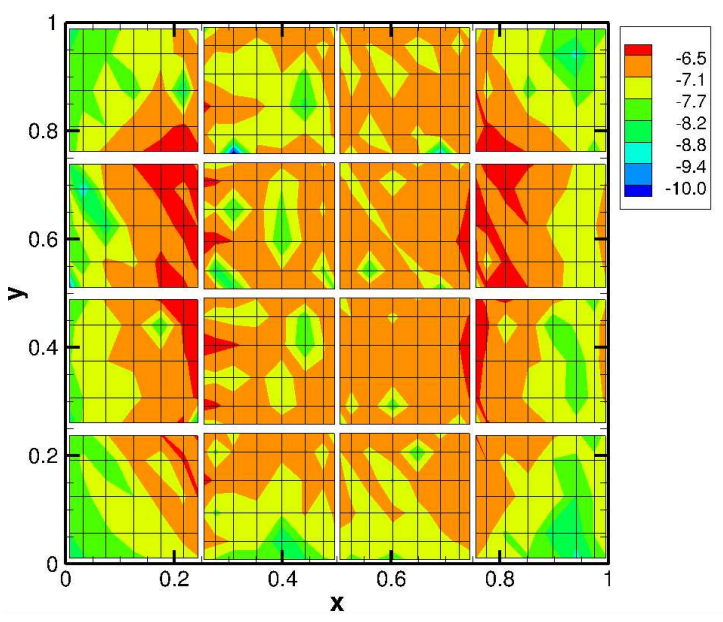

(d) $\tau_{\max }=10^{-6}$

Figure 5.7: Adaptation results for different required truncation errors (logarithmic scale); The computation Legendre-Gauss nodes are shown in each element. 


\subsection{Inviscid NACA0012 Airfoil}

A NACA0012 airfoil is simulated at an angle of attack $\alpha=0^{\circ}$ using the Euler equations and a Mach number $M=0.3$ (details in Table 5.1). Slip wall boundary conditions are set at all airfoil surfaces and start with meshes that are not symmetric. For unsymmetric meshes, the lift coefficient is not necessarily zero, but since a symmetric airfoil is considered at zero angle of attack, the numerical lift should tend towards zero as the mesh is refined. This test case reveals the advantages and limitations of the various adaptation strategies proposed. The NACA case is converged in time until the maximum norm of the residual equation falls below $10^{-8}$ using a uniform polynomial order $\mathrm{P}=8$, see Figure 5.8.

\begin{tabular}{|l|l|}
\hline Chord length & $L_{C}=1$ \\
Domain size (circular) & $L_{R}=20$ \\
Elements & $\# E l=407$ \\
Polynomial order & $P=8$ \\
Degrees of Freedom & $D o F=14652$ \\
Reynolds number & $R e=500$ \\
\hline
\end{tabular}

Table 5.1: NACA0012 simulation properties

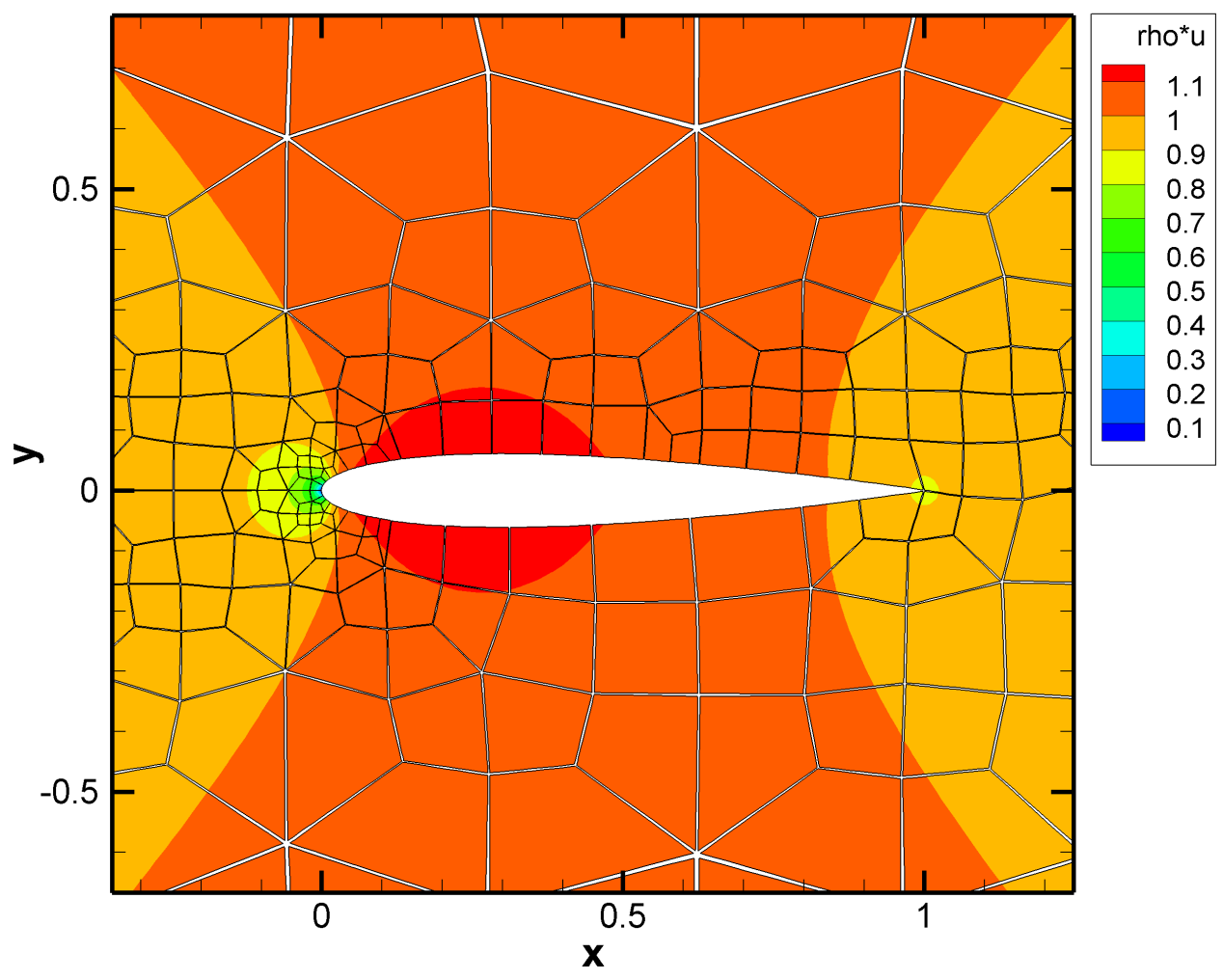

Figure 5.8: NACA0012 simulation, $\rho u$.

First, Figure 5.9 depicts the adapted meshes obtained when using the discretisation 
error based adaptation approach (or "feature based") with thresholds $10^{-3}$ (top) and $10^{-4}$ (bottom). It can be seen that the discretisation error provides a fine mesh at the leading edge and a coarser resolution at the trailing edge.
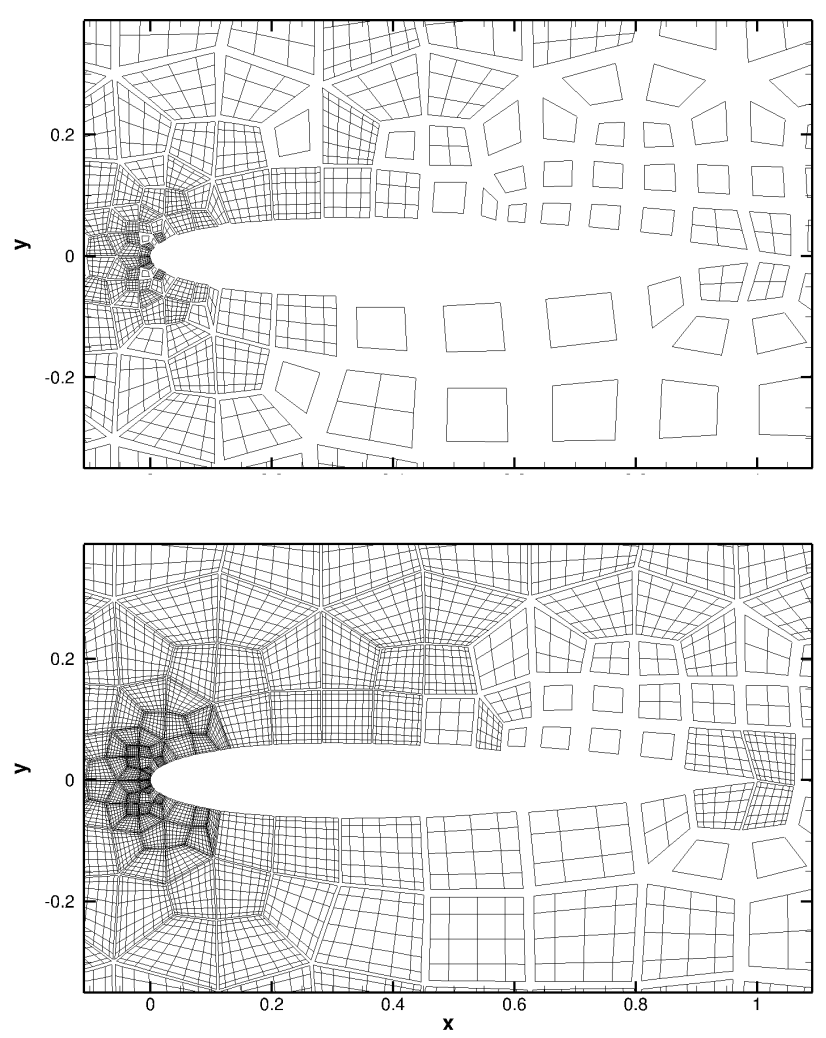

Figure 5.9: Adapted meshes using the discretisation error ("feature based") strategy with thresholds $10^{-2}$ (top) and $10^{-3}$ (bottom).

Second, the adapted meshes resulting from using the truncation error adaptations, Algorithm 5, are shown in Figure 5.10. The left side of the figure shows the adaptation result when using the truncation error while the right side shows the results based on the isolated truncation error for a threshold $10^{-4}$ (top) and $10^{-5}$ (bottom). It can be seen that both truncation based adaptation strategies (non-isolated and isolated) provide meshes with a high concentration of degrees of freedom near the leading and the trailing edge. In Figure 5.11 the three refinement criteria are compared using thresholds that provide similar number of DoF in the adapted meshes. It can be noticed that the discretisation error based adaptation provides a coarser mesh near the trailing edge whilst similar meshes are obtained near the leading edge.

Figure 5.12 summarises lift and drag coefficients against the global number of degrees of freedom (DoF) using the various adaptation strategies. The adaptation thresholds are maintained for all methods to $10^{-2}, 10^{-3}, 10^{-4}$ and $10^{-5}$. Uniformly adapted meshes are included as a reference. The figure shows convergence for the lift (left) and drag (right) in all cases once the asymptotic behaviour is reached (i.e. small enough required thresholds). 

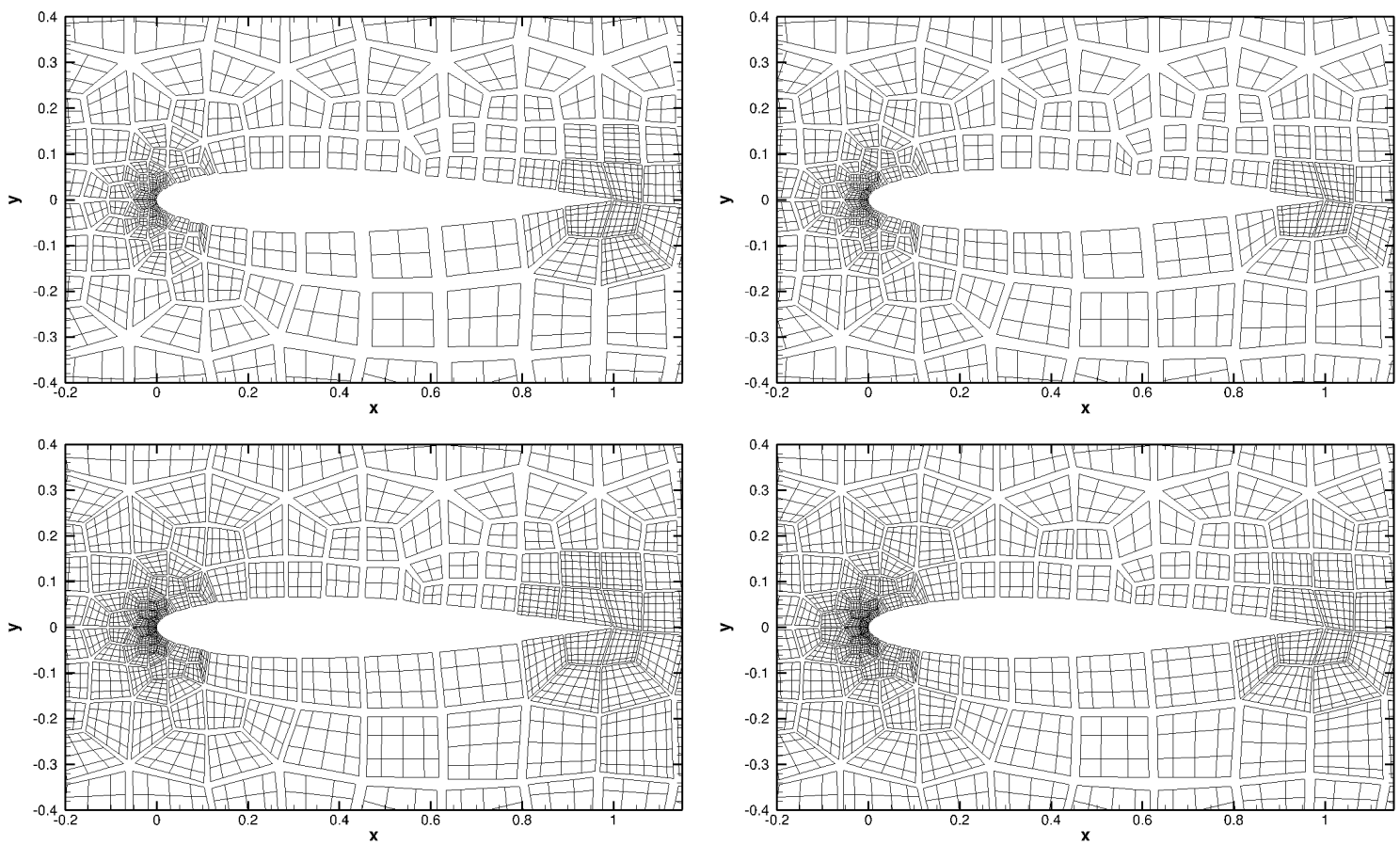

Figure 5.10: Adapted meshes based on truncation error, TE (left) and isolated truncation error, ITE (right) for thresholds $10^{-4}$ (top) and $10^{-5}$ (bottom).

Firstly, comparing the discretisation and truncation error adaptations shows that the truncation error requires fewer number of DoF to attain similar accuracy in the selected output functionals. Secondly, comparing truncation error to isolated truncation error adaptation shows that the isolated version requires fewer DoF than the non-isolated version. Thirdly, comparing isotropic to anisotropic adaptation strategies (for both truncation and isolated truncation) shows a clear reduction of overall number of degrees of freedom to attain similar levels of accuracy. In summary, for a similar level of accuracy, i.e. $\left|\frac{C_{D}^{\text {Adapted }}-C_{D}^{P=8}}{C_{D}^{P=8}}\right|<0.2$, the following results are obtained:

- For the discretisation error based adaptation $48 \%$ reduction of DoF compared to the uniform refinement

- For the truncation error based adaptation a 70\% reduction of DoF compared to the uniform refinement

- A $10 \%$ reduction of the anisotropic truncation error compared to the isotropic approach

- For the isolated truncation error based adaptation a $84 \%$ reduction of DoF compared to the uniform refinement

- A $16 \%$ reduction of the anisotropic isolated truncation error compared to the isotropic approach. 

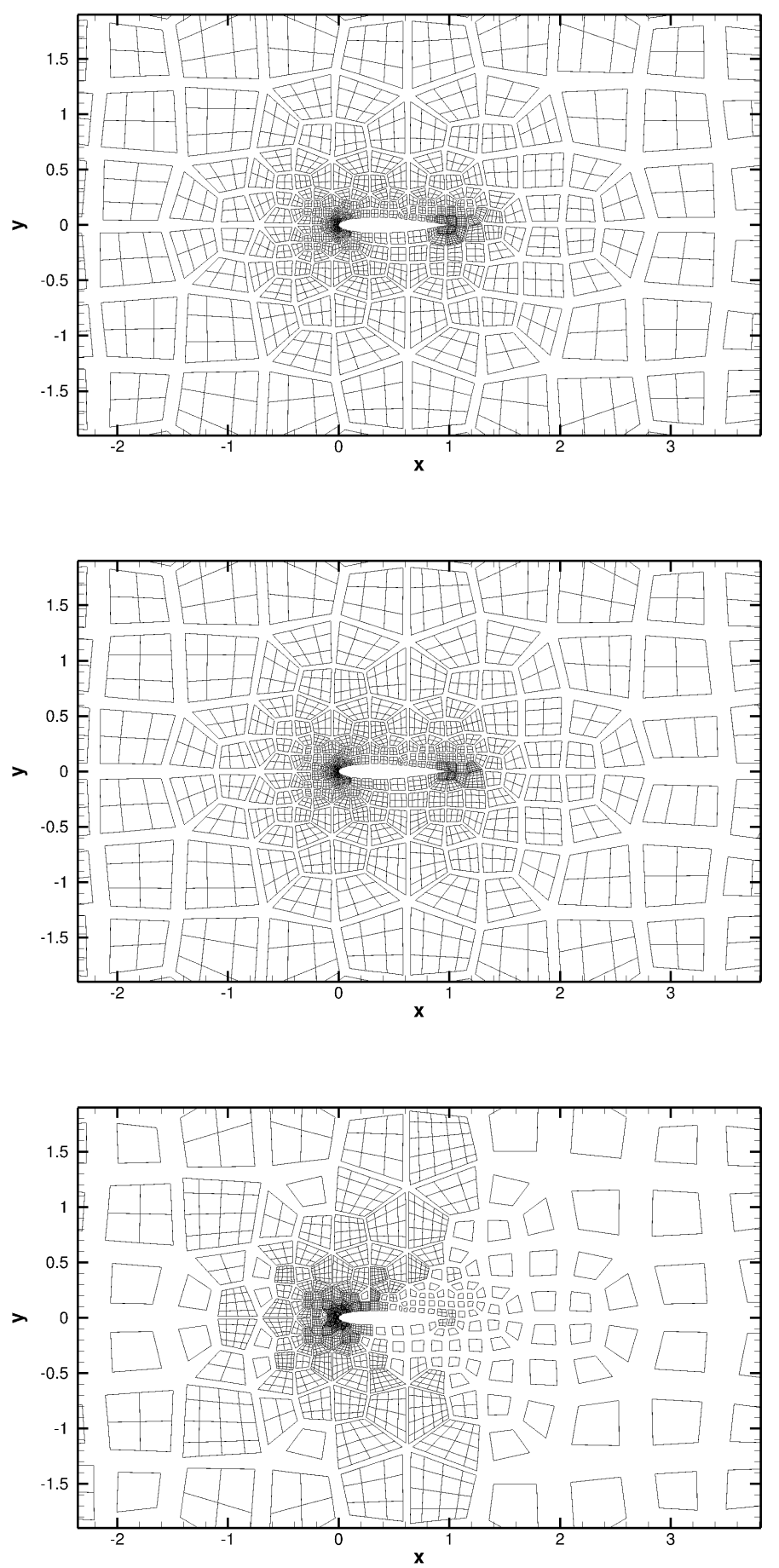

Figure 5.11: Adapted meshes based on different refinement criteria with comparable final number of DoF. Top is anisotropic truncation error based $\left(10^{-4}\right.$ threshold, 20480 DoF $)$. Middle is anisotropic isolated truncation error based $\left(10^{-5}\right.$ threshold, $\left.21444 \mathrm{DoF}\right)$. Bottom is isotropic discretisation error based $\left(10^{-2}\right.$ threshold, $\left.19096 \mathrm{DoF}\right)$.

The results for all simulations can be seen in Table 5.2.

This test case shows that the anisotropic isolated truncation error criteria outperforms 


\begin{tabular}{|c|c|c|c|c|c|c|c|}
\hline Simulation & $\mathrm{TE}$ & ITE & $\mathrm{DE}$ & $\tau_{\max }$ & DoF & $c_{D}$ & $c_{L}$ \\
\hline Uniform $\mathrm{P}=2$ & & & & - & 14652 & $8.03 \cdot 10^{-3}$ & $9.28 \cdot 10^{-4}$ \\
\hline Uniform $\mathrm{P}=3$ & & & & - & 26048 & $5.79 \cdot 10^{-3}$ & $1.06 \cdot 10^{-3}$ \\
\hline Uniform $\mathrm{P}=4$ & & & & - & 40700 & $4.53 \cdot 10^{-3}$ & $8.68 \cdot 10^{-4}$ \\
\hline Uniform $\mathrm{P}=5$ & & & & - & 58608 & $3.73 \cdot 10^{-3}$ & $6.64 \cdot 10^{-4}$ \\
\hline Uniform $\mathrm{P}=6$ & & & & - & 79772 & $3.18 \cdot 10^{-3}$ & $5.08 \cdot 10^{-4}$ \\
\hline Uniform $\mathrm{P}=7$ & & & & - & 104192 & $2.76 \cdot 10^{-3}$ & $3.98 \cdot 10^{-4}$ \\
\hline Uniform $\mathrm{P}=9$ & & & & - & 131686 & $2.45 \cdot 10^{-3}$ & $2.62 \cdot 10^{-4}$ \\
\hline Isotropic TE & $\mathrm{X}$ & & & $10^{-2}$ & 6552 & $6.77 \cdot 10^{-3}$ & $1.47 \cdot 10^{-3}$ \\
\hline Isotropic TE & $\mathrm{X}$ & & & $10^{-3}$ & 10824 & $2.51 \cdot 10^{-3}$ & $7.55 \cdot 10^{-4}$ \\
\hline Isotropic TE & $\mathrm{X}$ & & & $10^{-4}$ & 21444 & $2.38 \cdot 10^{-3}$ & $9.32 \cdot 10^{-4}$ \\
\hline Isotropic TE & $\mathrm{X}$ & & & $10^{-5}$ & 43540 & $2.37 \cdot 10^{-3}$ & $5.19 \cdot 10^{-4}$ \\
\hline Isotropic TE & $\mathrm{X}$ & & & $10^{-6}$ & 66096 & $2.49 \cdot 10^{-3}$ & $3.50 \cdot 10^{-4}$ \\
\hline Isotropic ITE & & $\mathrm{X}$ & & $10^{-2}$ & 6652 & $3.04 \cdot 10^{-3}$ & $2.42 \cdot 10^{-2}$ \\
\hline Isotropic ITE & & $\mathrm{X}$ & & $10^{-3}$ & 13448 & $9.50 \cdot 10^{-4}$ & $1.17 \cdot 10^{-3}$ \\
\hline Isotropic ITE & & $\mathrm{X}$ & & $10^{-4}$ & 25460 & $2.27 \cdot 10^{-3}$ & $5.50 \cdot 10^{-4}$ \\
\hline Isotropic ITE & & $\mathrm{X}$ & & $10^{-5}$ & 42764 & $2.49 \cdot 10^{-3}$ & $4.13 \cdot 10^{-4}$ \\
\hline Isotropic ITE & & $\mathrm{X}$ & & $10^{-5}$ & 62388 & $2.48 \cdot 10^{-3}$ & $4.24 \cdot 10^{-4}$ \\
\hline Isotropic DE & & & $\mathrm{X}$ & $10^{-1}$ & 6532 & $1.51 \cdot 10^{-2}$ & $2.17 \cdot 10^{-3}$ \\
\hline Isotropic DE & & & $\mathrm{X}$ & $10^{-2}$ & 19096 & $8.74 \cdot 10^{-3}$ & $1.35 \cdot 10^{-3}$ \\
\hline Isotropic DE & & & $\mathrm{X}$ & $10^{-3}$ & 67936 & $8.57 \cdot 10^{-4}$ & $3.23 \cdot 10^{-4}$ \\
\hline Isotropic DE & & & $\mathrm{X}$ & $10^{-4}$ & 99160 & $2.67 \cdot 10^{-3}$ & $3.31 \cdot 10^{-4}$ \\
\hline Isotropic DE & & & $\mathrm{X}$ & $10^{-5}$ & 118408 & $2.43 \cdot 10^{-3}$ & $3.21 \cdot 10^{-4}$ \\
\hline Isotropic DE & & & $\mathrm{X}$ & $10^{-6}$ & 125972 & $2.45 \cdot 10^{-3}$ & $3.20 \cdot 10^{-4}$ \\
\hline Anisotropic TE & $\mathrm{X}$ & & & $10^{-2}$ & 6528 & $1.41 \cdot 10^{-2}$ & $8.09 \cdot 10^{-4}$ \\
\hline Anisotropic TE & $\mathrm{X}$ & & & $10^{-3}$ & 10836 & $2.65 \cdot 10^{-3}$ & $1.89 \cdot 10^{-3}$ \\
\hline Anisotropic TE & $\mathrm{X}$ & & & $10^{-4}$ & 20480 & $1.38 \cdot 10^{-3}$ & $1.30 \cdot 10^{-3}$ \\
\hline Anisotropic TE & $\mathrm{X}$ & & & $10^{-5}$ & 39160 & $2.18 \cdot 10^{-3}$ & $5.14 \cdot 10^{-4}$ \\
\hline Anisotropic TE & $\mathrm{X}$ & & & $10^{-6}$ & 61644 & $2.3 \cdot 10^{-4}$ & $3.81 \cdot 10^{-4}$ \\
\hline Anisotropic ITE & & $\mathrm{X}$ & & $10^{-2}$ & 6568 & $1.01 \cdot 10^{-2}$ & $2.81 \cdot 10^{-3}$ \\
\hline Anisotropic ITE & & $\mathrm{X}$ & & $10^{-3}$ & 10824 & $2.21 \cdot 10^{-3}$ & $1.65 \cdot 10^{-3}$ \\
\hline Anisotropic ITE & & $\mathrm{X}$ & & $10^{-4}$ & 21444 & $2.24 \cdot 10^{-3}$ & $4.91 \cdot 10^{-4}$ \\
\hline Anisotropic ITE & & $\mathrm{X}$ & & $10^{-5}$ & 38688 & $2.41 \cdot 10^{-3}$ & $4.41 \cdot 10^{-4}$ \\
\hline Anisotropic ITE & & $\mathrm{X}$ & & $10^{-6}$ & 38688 & $2.40 \cdot 10^{-3}$ & $4.41 \cdot 10^{-4}$ \\
\hline
\end{tabular}

Table 5.2: NACA0012 simulations and DoF.

the other criteria considered for adaptation.

Finally, according to Wang et al. [Wang 2013], who summarised results from various high order solvers for a NACA0012 at $\alpha=2^{\circ}$, the high-order asymptotic convergence cannot be attained when using uniform refinement at the trailing edge of the airfoil. Indeed, the airfoil trailing edge results in a flow discontinuity that limits the solution smoothness and hence the exponential convergence when enriching the polynomial solution space. The truncation error has been proven to scale well for cases in the asymptotic range (see Rubio et al. [Rubio 2014]) while limitations have been observed for problems with singularities. In such cases h-refinement may enhance the solution accuracy as compared to adaptation strategies restricted to p-refinement based on the truncation error, which nonetheless have shown to perform well even for this challenging test case. 

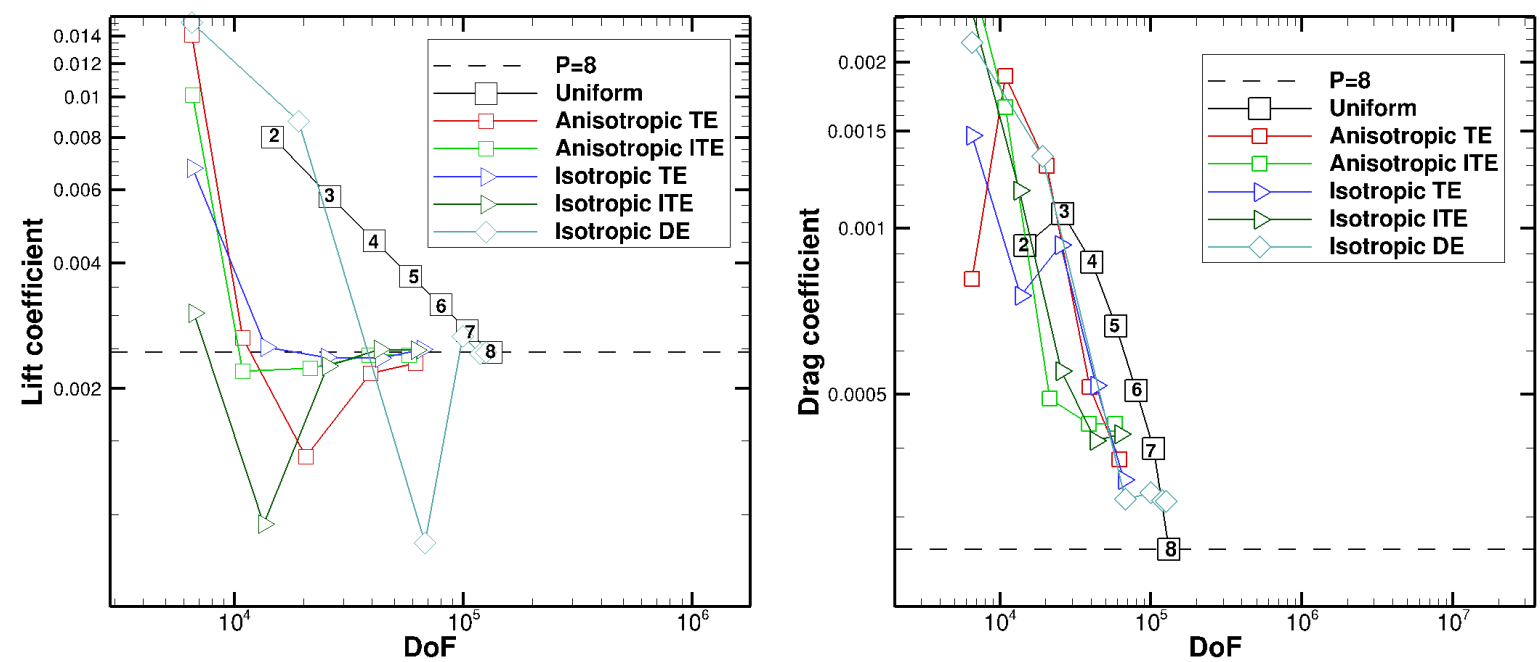

Figure 5.12: Lift and drag coefficients for NACA0012 based on different adaptation algorithms. Uniform refinement, Discretisation error (DE), Truncation error (TE) and Isolated Truncation error (ITE) adaptation strategies are shown.

\subsection{Boundary Layer Test Case}

The previous section proved the favourable properties of the truncation error based adaptation strategies. It was shown that the error estimated agrees well with the exact error for the manufactured solution test case.

We now turn our attention to a more complex application with higher number of degrees of freedom i.e. a boundary layer above a flat plate. To simulate the viscous compressible Navier-Stokes equations, the DGSEM method is modified to account for viscous effects. To this end, an Interior Penalty method as detailed in Arnold et al. [Arnold 2001] is incorporated. For this purpose a boundary layer simulation is performed for Reynolds number per unit length $\mathrm{Re}=500$ and Mach number $\mathrm{M}=0.2$. The governing equations are the viscous compressible Navier-Stokes equations, written in non-dimensional form:

$$
\mathbf{Q}_{t}+\mathbf{F}_{x}^{a}+\mathbf{G}_{y}^{a}=\frac{1}{R e}\left(\mathbf{F}_{x}^{v}+\mathbf{G}_{y}^{v}\right)
$$

where $\mathbf{Q}$ is $(\rho, \rho u, \rho v, \rho e)^{T}, \mathbf{F}_{x}^{a}$ and $\mathbf{G}_{y}^{a}$ are the advective fluxes, defined as,

$$
\mathbf{F}^{a}=\left[\begin{array}{c}
\rho u \\
p+\rho u^{2} \\
\rho u v \\
u(\rho e+p)
\end{array}\right], \mathbf{G}^{a}=\left[\begin{array}{c}
\rho v \\
\rho u v \\
p+\rho v^{2} \\
v(\rho e+p)
\end{array}\right]
$$

The pressure $p$ is defined through the ideal gas equation. On the other hand, the diffusive 
fluxes are defined as

$$
\mathbf{F}^{v}=\left[\begin{array}{c}
0 \\
\tau_{x x} \\
\tau_{x y} \\
u \tau_{x x}+v \tau_{x y}+\frac{\kappa}{(\gamma-1) \operatorname{Pr} M^{2}} T_{x}
\end{array}\right], \mathbf{G}^{v}=\left[\begin{array}{c}
0 \\
\tau_{y x} \\
\tau_{y y} \\
u \tau_{y x}+v \tau_{y y}+\frac{\kappa}{(\gamma-1) \operatorname{Pr} M^{2}} T_{y}
\end{array}\right]
$$

and the Stokes hypothesis

$$
\begin{aligned}
\tau_{x x} & =2 \mu\left(u_{x}-\left(u_{x}+v_{y}\right) / 3\right), \\
\tau_{y y} & =2 \mu\left(v_{y}-\left(u_{x}+v_{y}\right) / 3\right), \\
\tau_{x y} & =\tau_{y x}=\mu\left(v_{x}+u_{y}\right),
\end{aligned}
$$

where $T$ the temperature, $\mu$ is the viscosity, $\gamma$ is the heat capacity ratio, $\kappa$ is the thermal diffusivity and the non-dimensional parameters $R e$ the Reynolds number, $P r$ the Prandtl number and $M$ the Mach number. The Prandtl number and the heat capacity ratio are set to the usual values for air $\operatorname{Pr}=0.72$ and $\gamma=1.4$. The viscosity, $\mu$, and the thermal diffusivity, $\kappa$ are calculated using Sutherland's law.

\begin{tabular}{|l|l|}
\hline Boundary Length & $L_{B}=10$ \\
Singularity & $L_{S}=4$ \\
Elements & $\# E l=50$ \\
Polynomial order & $P=8$ \\
Degrees of Freedom & $D o F=1800$ \\
Mach number & $M=0.2$ \\
Reynolds number & $R e=500$ \\
\hline
\end{tabular}

Table 5.3: Boundary Layer Simulation overview.

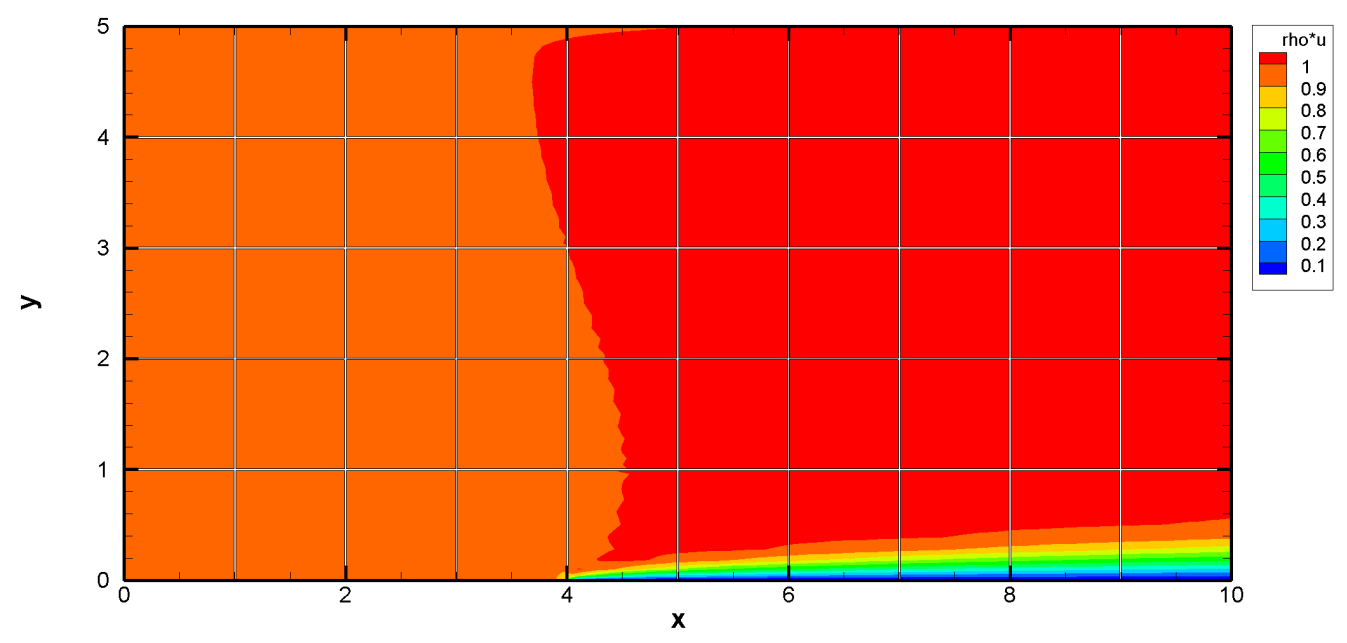

Figure 5.13: Boundary layer simulation $\rho u$ with singularity at $x=4$. 
Uniform boundary conditions are used at the inflow, while at the bottom boundary a symmetric boundary condition is used up to the stagnation point located at $x=4$ and an adiabatic wall assumed further downstream. Pressure exit boundary conditions are applied for the outflow and the far field. A steady solution is calculated with $P_{x}=P_{y}=8$ and converged to a residual of $10^{-10}$. The estimation for the truncation error for $\tau_{8}^{7}$ is shown in Figure 5.14.

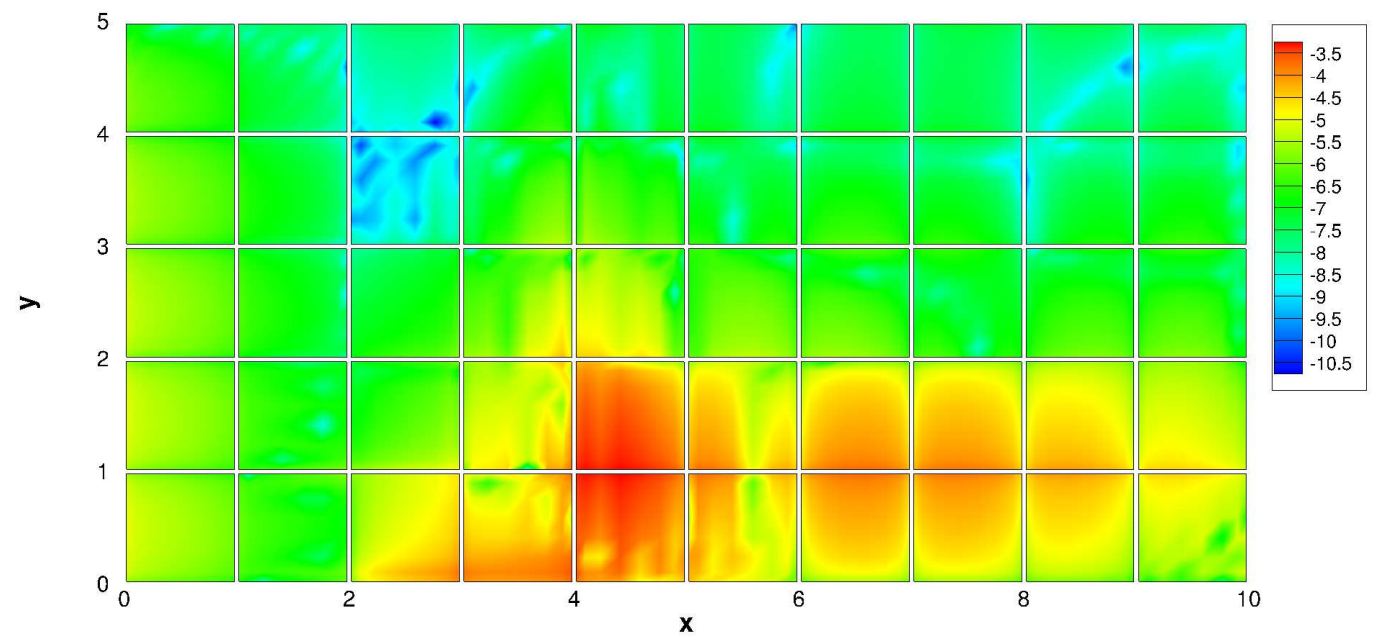

Figure 5.14: Truncation error $\tau_{8}^{7}$ based on the density (logarithmic scale), for boundary layer simulation, singularity at $x=4$.

As expected, the truncation error is large around the singularity at $x=4$ and close to the wall in the downstream region. Certainly, this is the region that contains complex flow features and consequently requires higher resolution e.g. needs to be adapted using a higher polynomial order. Furthermore, the error is slightly higher at the inlet than in the outer region due to the imposed inflow boundary conditions and the interaction with the singularity which could be avoided by extending the distance between the inflow and the begin of the boundary layer.

\subsubsection{Adaptation}

The adaptation process is applied to the boundary layer test case using a mesh of $5 \times 10$ elements and an initial polynomial order of $P_{x}=P_{y}=P=8$. Now, only the quasia priori estimation process without the correction term is detailed since no significant differences are observed when adding the correction. The main differences appear in the computational costs, which will be explained later in section 5.5.

First the effect of varying the truncation error threshold $\left(\tau_{\max }\right)$ is explored. In Figure 5.15 three meshes are shown where the threshold levels are varied: Figure 5.15 (top-left) $\tau_{\max }=10^{-1}$, Figure 5.15 (top-right) $\tau_{\max }=10^{-3}$ and Figure 5.15 (bottom) $\tau_{\max }=10^{-2}$. Comparing these meshes it can be seen that decreasing the threshold level results in finer 
meshes. More interesting is the clear anisotropic refinement enabled by the high order discontinuous Galerkin discretization as shown in Figure 5.15 (bottom). Note, that when selecting $\tau_{\max }=10^{-3}$, Figure 5.15 (top-right), the maximum allowable polynomial $P=10$ is reached and hence the anisotropic refinements is masked.

Having shown the effect of varying the truncation error threshold, the threshold of $\tau_{\max }=10^{-2}$ can be retained, mesh depicted in Figure 5.15 (bottom). This threshold implies that the convergence is stopped when the simulation reaches a residual of $10^{-3}$ (i.e. $F=10$ in section 4.3 ).
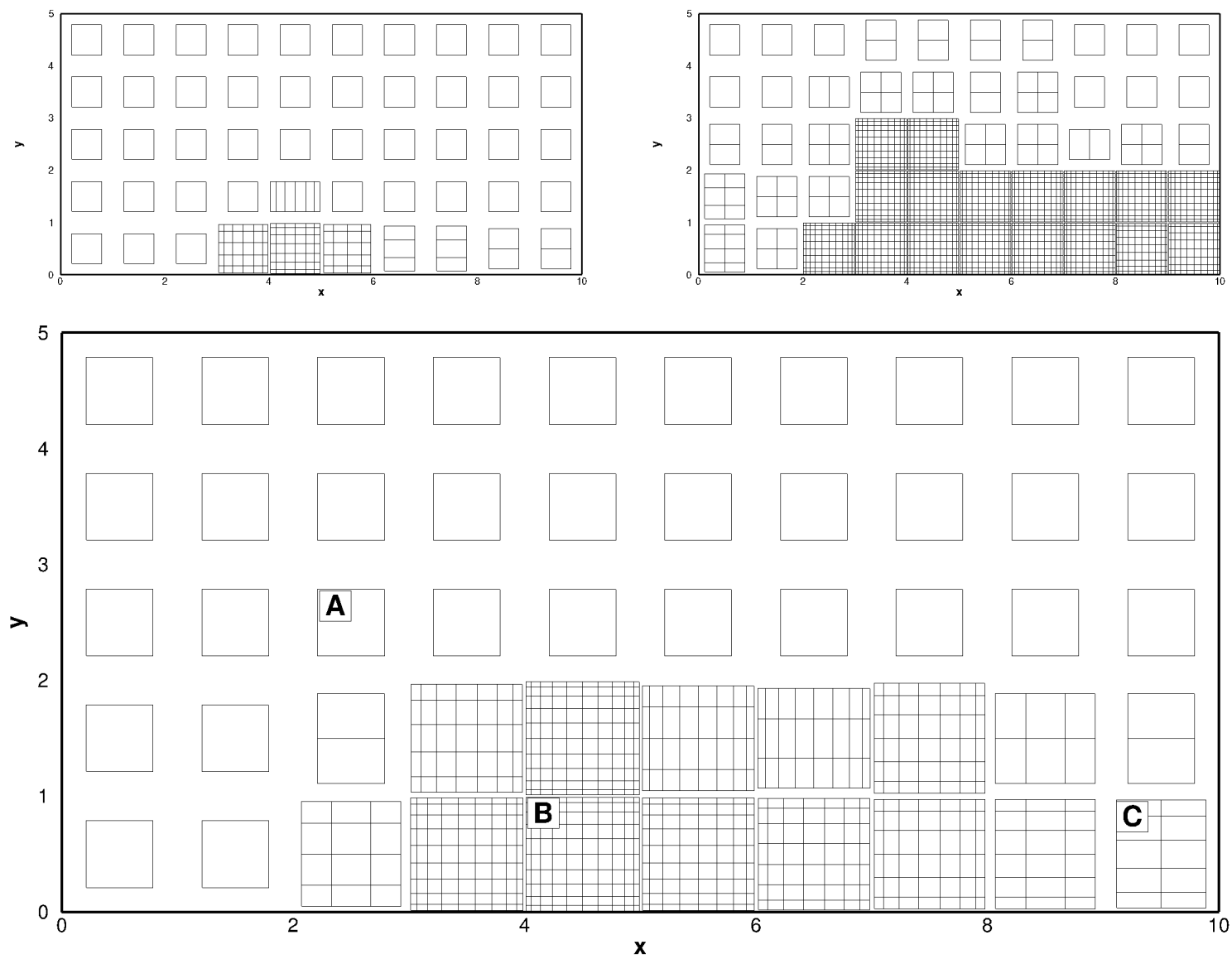

Figure 5.15: Adapted boundary layer meshes based on various truncation error thresholds: $10^{-1}$ (upper-left), $10^{-2}$ (lower-left) and $10^{-3}$ (upper-right). The computation LegendreGauss nodes are shown in each element.

Following Algorithm 4, the estimates are calculated for $N_{x}, N_{y}=1, \ldots, 7$. This estimation is shown in Figure 5.16 for three particular elements A, B and C, depicted in Figure 5.15 (bottom). The first plot Figure 5.16 (top) shows the adaptation for element A, which is located far from the leading edge singularity. In this case the estimates for the different polynomial orders show that a polynomial order of 1 suffices to reach the desired threshold $\tau_{\text {max }}=10^{-2}$. Figure 5.16 (bottom), considers Element $\mathrm{C}$ (located near the outflow and the wall), these error estimates show the anisotropic character expected from a mesh element located near the wall, where large gradients in the wall-normal ( $y$-direction) dominate. In 
this case the polynomial order that fulfills the truncation threshold is $P_{x}=2$ and $P_{y}=6$. Depending on the test case it may happen that the estimates of certain elements do not behave accordingly to the asymptotic range. This is the case for element B (located near the boundary layer singularity or leading point). Figure 5.15 shows that this element has a high error due to its location very close to the leading edge singularity. To resolve this type of elements, the extrapolation procedure as depicted in 5.16 (center) is followed. If the extrapolated value provides a polynomial order that is below the maximum allowable polynomial order then the extrapolated order is selected (this is the case of the red curve or $y$-direction). However, if the extrapolation shows that a polynomial higher than possible (above the allowable maximum) is needed, the maximum polynomial order is set for this element direction ( $\mathrm{P}=10$ in this case). Another possibility would be to recalculate the entire underlying simulation with a higher polynomial order or to refine the area around the singularity with smaller elements (h-refinement).

Finally and for completeness, Figure 5.17 shows the relative error for the drag coefficient, $\left|c_{D}-c_{D(P=10)}\right| /\left|c_{D(P=10)}\right|$ for the boundary layer case. It can be seen that when decreasing the truncation error thresholds (values within the boxes for the red line) the relative error for the drag decreases. In addition, drag-errors issued from non-adapted meshes where a uniform polynomial order is used (blue line and boxes show the polynomial order) are included. When comparing the drag-errors issued from the p-adapted meshes to the errors obtained using uniform polynomial, it can be seen that for the same number of degrees of freedoms, the error is lower if the anisotropic adaptation based on tau-estimates is used. These last results show that, at least for the boundary layer test case, the accuracy of the drag is governed by the truncation error.

\subsubsection{Adaptation Strategy Comparison}

In this part the adaptation strategies are compared for the different input quantities (truncation error, isolated truncation error, discretion error) for the viscous Navier-Stokes simulation. The flat plate boundary layer case was simulated under the same conditions like stated in the section before. The Reynolds number per unit length is $\operatorname{Re}=500$ and the Mach number $\mathrm{M}=0.2$. The length of the plate is $L_{x}=6$ and the leading edge singularity is included in the simulation (and is located at the point $(x, y)=(4,0)$ ). The finest simulation used a uniform polynomial order $P=8$ and was converged until the equation residual fell below $10^{-10}$.

Using the discretisation error based adaptation method results in the meshes in Figure 5.18. For this adaptation the thresholds $10^{-1}, 10^{-2}, 10^{-3}$ are depicted. Besides a high refinement around the singularity, which is common to all the schemes, it can be seen that this approach also refines the far field.

Applying the adaptation based on the truncation error and the isolated truncation error results in the meshes depicted in Figure 5.19. The focus of this comparison lies on the reduction of DoF. Accordingly Algorithm 5 was used for the comparison. Here, the 
same thresholds as before $\left(10^{-1}, 10^{-2}, 10^{-3}\right)$ are used for adaptation. The truncation error shows a higher refinement around the singularity in comparison to the isolated truncation error. This results in fewer overall number of DoF for the isolated truncation error case than for the truncation error case when the same threshold is considered. Both truncation error based adaptions provide meshes with fewer DoF than if the discretisation error (feature based) approach is used.

Finally, in Figure 5.20 the drag coefficient $C_{D}$ is depicted, provided by the adapted meshes and compared to the solutions using uniform polynomials. It should be noted that the maximum polynomial order for all adapted meshes is set to $P^{n e w}=8$, such that this can be considered as the optimal solution.

The approaches based on the truncation error achieve similar drag coefficients with fewer global number of DoF than when using uniform adaptation or the discretisation error ("feature based") strategy. When using the discretisation error approach, the refinement in the far field (see Figure 5.18) results in a large number of DoF that are not required to improve the accuracy in the drag coefficient, which leads to an oversized mesh.

The anisotropic character of the boundary layer solution provides anisotropic meshes that significantly reduce the number of DoF whilst maintaining a good level of accuracy.

In summary, for a similar level of accuracy, i.e. $\left|\frac{C_{D}^{\text {Adapted }}-C_{D}^{P=10}}{C_{D}^{P=10}}\right|<0.1$, the following results are obtained:

- For the discretisation error based adaptation a $25 \%$ reduction of DoF compared to the uniform refinement

- For the truncation error based adaptation a $84 \%$ reduction of DoF compared to the uniform refinement and a $27 \%$ reduction of the anisotropic truncation error compared to the isotropic approach

- For the isolated truncation error based adaptation a 85\% reduction of DoF compared to the uniform refinement and

- A $16 \%$ reduction of the anisotropic isolated truncation error compared to the isotropic approach.

See Table 5.4 for all the values.

For the boundary layer case it can be assumed that the key point of an overall high accuracy is a high resolution in the direction normal to the wall. Figure 5.21 shows the boundary layer profile of $\rho u$ in wall-normal direction, $x=7.5$ and the corresponding node distribution for $\tau_{P}^{N}$ for $N=1,2,3,4$. It can clearly be seen that for lower truncation error more nodes are necessary in the areas where the boundary layer profile shows steeper gradients. 


\begin{tabular}{|l||l|l|l|l|l|l|}
\hline Simulation & $\mathrm{TE}$ & $\mathrm{ITE}$ & $\mathrm{DE}$ & $\tau_{\max }$ & $\mathrm{DoF}$ & $c_{D}$ \\
\hline \hline Uniform P=2 & & & & & 1800 & 0.1783153352 \\
\hline Uniform P=3 & & & & & 3200 & 0.1735958858 \\
\hline Uniform P=4 & & & & & 5000 & 0.1617047791 \\
\hline Uniform P=5 & & & & & 7200 & 0.1592936552 \\
\hline Uniform P=6 & & & & & 9800 & 0.1564082704 \\
\hline Uniform P=7 & & & & & 12800 & 0.154008979 \\
\hline Uniform P=8 & & & & & 16200 & 0.1536315846 \\
\hline Anisotropic TE & $\mathrm{X}$ & & & $10^{-1}$ & 1356 & 0.1512499397 \\
\hline Anisotropic TE & $\mathrm{X}$ & & & $10^{-2}$ & 2464 & 0.1534748992 \\
\hline Anisotropic TE & $\mathrm{X}$ & & & $10^{-3}$ & 4340 & 0.1536272907 \\
\hline Anisotropic TE & $\mathrm{X}$ & & & $10^{-4}$ & 6004 & 0.153636067 \\
\hline Anisotropic ITE & & $\mathrm{X}$ & & $10^{-1}$ & 1260 & 0.154175549 \\
\hline Anisotropic ITE & & $\mathrm{X}$ & & $10^{-2}$ & 2356 & 0.1535148012 \\
\hline Anisotropic ITE & & $\mathrm{X}$ & & $10^{-3}$ & 3760 & 0.1536109168 \\
\hline Anisotropic ITE & & $\mathrm{X}$ & & $10^{-4}$ & 6104 & 0.1536345934 \\
\hline Isotropic TE & $\mathrm{X}$ & & & $10^{-1}$ & 1736 & 0.1512499397 \\
\hline Isotropic TE & $\mathrm{X}$ & & & $10^{-2}$ & 3372 & 0.1533841336 \\
\hline Isotropic TE & $\mathrm{X}$ & & & $10^{-3}$ & 4524 & 0.1536275362 \\
\hline Isotropic TE & $\mathrm{X}$ & & & $10^{-4}$ & 6700 & 0.1536287669 \\
\hline Isotropic ITE & & $\mathrm{X}$ & & $10^{-1}$ & 1844 & 0.154175549 \\
\hline Isotropic ITE & & $\mathrm{X}$ & & $10^{-2}$ & 2800 & 0.1534778027 \\
\hline Isotropic ITE & & $\mathrm{X}$ & & $10^{-3}$ & 4160 & 0.1535983248 \\
\hline Isotropic ITE & & $\mathrm{X}$ & & $10^{-4}$ & 6384 & 0.1536338491 \\
\hline Isotropic DE & & & $\mathrm{X}$ & $10^{-2}$ & 1056 & 0.2069358353 \\
\hline Isotropic DE & & & $\mathrm{X}$ & $10^{-3}$ & 2432 & 0.1597543553 \\
\hline Isotropic DE & & & $\mathrm{X}$ & $10^{-4}$ & 7032 & 0.1543239734 \\
\hline Isotropic DE & & & $\mathrm{X}$ & $10^{-5}$ & 12188 & 0.1535630725 \\
\hline Isotropic DE & & & $\mathrm{X}$ & $10^{-6}$ & 15372 & 0.1536329167 \\
\hline
\end{tabular}

Table 5.4: Boundary Layer simulation results.

\subsubsection{Reynolds Number Variations}

A variation of the Reynolds number influences the adaptation process and varies the results. Using a higher Reynolds number and keeping the other flow and geometry properties constant, leads to sharper gradients in the wall-normal downstream velocity profiles. To resolve these profiles finer meshes (higher polynomial order and more nodes) are necessary. Figure 5.22 shows the adaptations for the boundary layer for three different Reynolds numbers: $R e=100,500,1000$. The displayed results are for the truncation error with a threshold of $\tau_{\max }=10^{-2}$. It can be seen that the profiles related to $R e=1000$ have a higher refinement closer to the wall in comparison to the simulations with low Reynolds numbers. The adaptation results for the isolated truncation error are shown in Figure 5.23. It can be seen that there is generally less refinement at the elements close to the boundary layer, which comes from the fact that only the volume contributions and not the surface fluxes (which tend to be higher for higher Reynolds numbers) are considered to evaluate the error. 


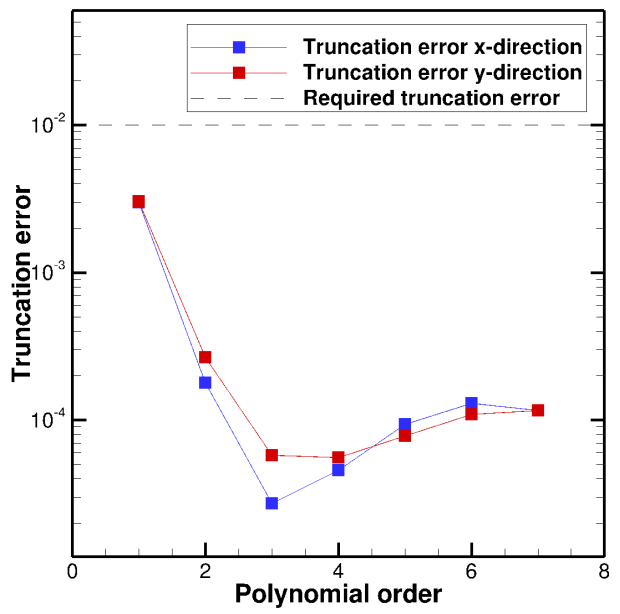

(a) Estimates for element A

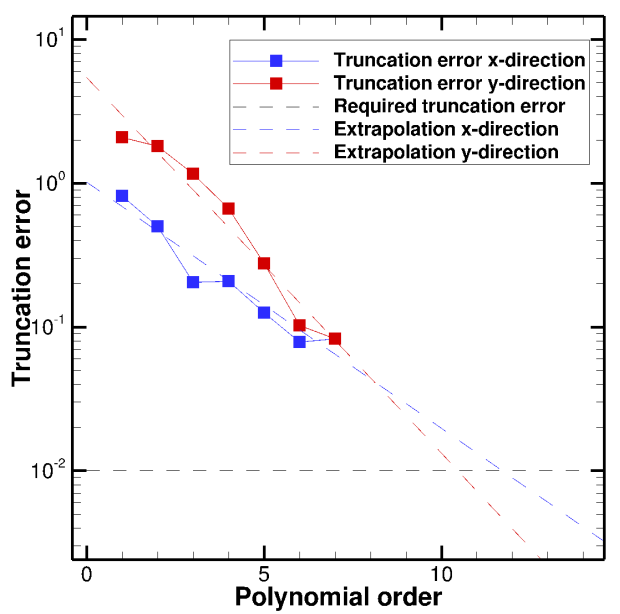

(b) Estimates for element B

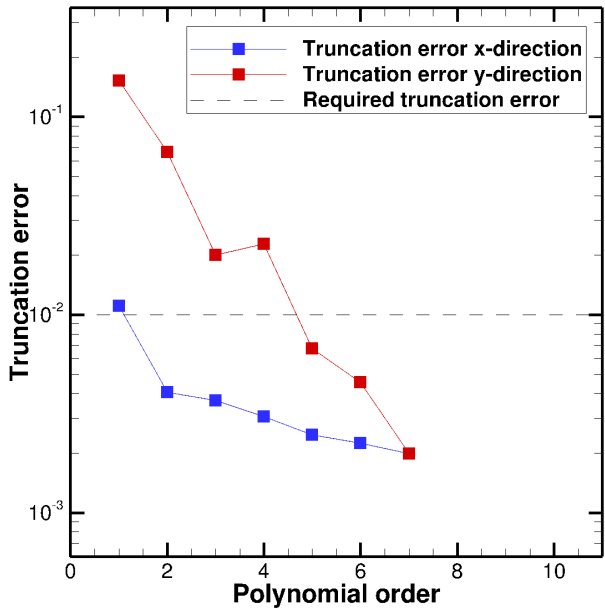

(c) Estimates for element C

Figure 5.16: Error estimation and required error for elements A (top), B (middle) and C (bottom) of Figure 5.15, linear error extrapolation is used on elements B and C. Horizontal dashed line shows the $10^{-3}$ threshold. 


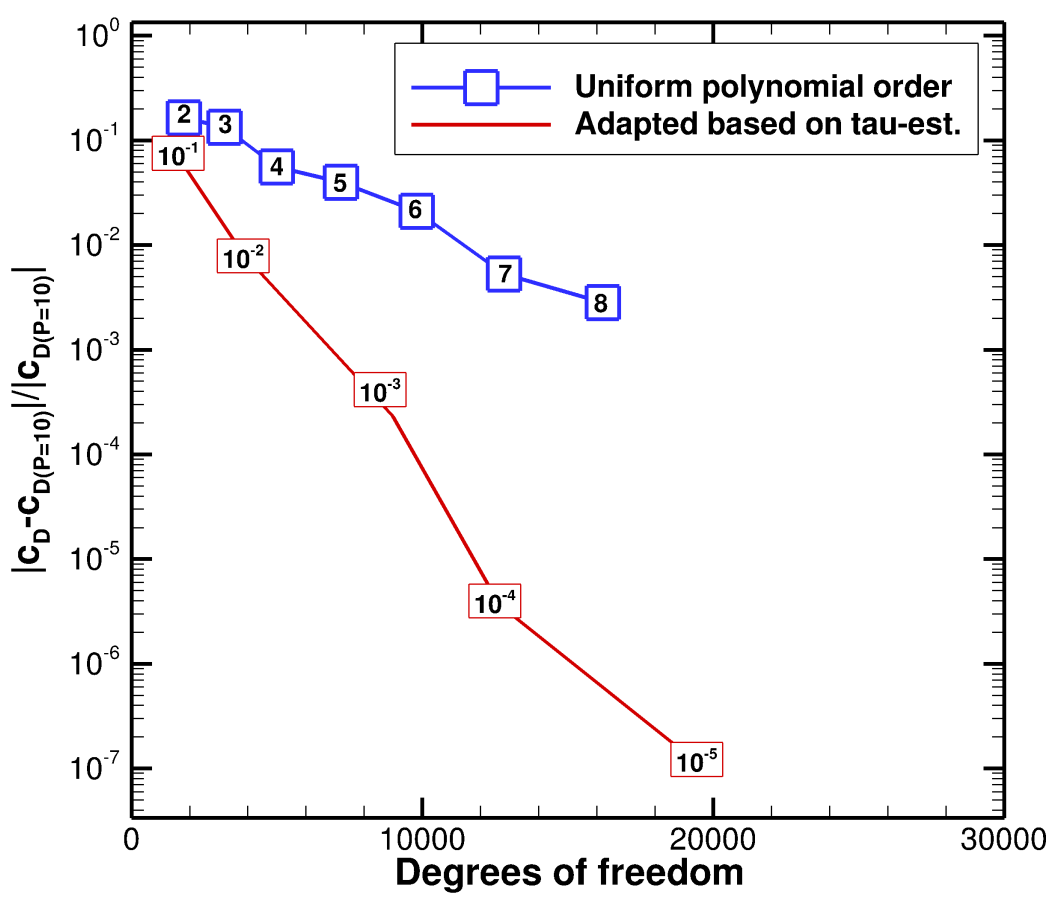

Figure 5.17: $c_{D}$ error based on different simulations on the corresponding DoF; uniform polynomial order solutions (blue with polynomial order in the box) and solutions obtained by the $\tau$-truncation error adaptation process (red with the adaptation criterion $\tau_{\max }$ in the box); Plotted value $\frac{\left|c_{D}-c_{D(P=10)}\right|}{\left|c_{D(P=10)}\right|}$, where $c_{D(P=10)}$ is calculated on a uniformly refined mesh with $P=10$. 

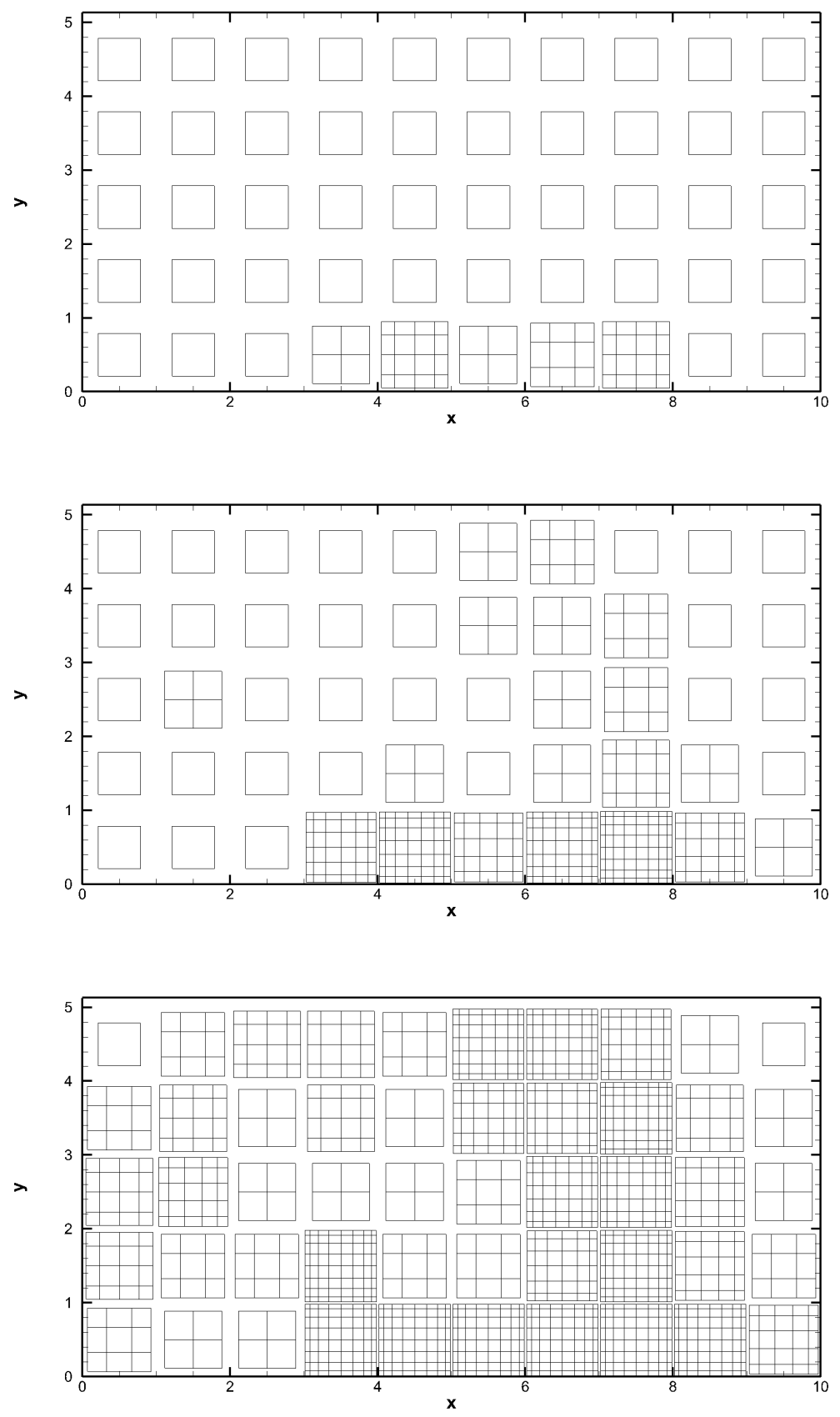

Figure 5.18: Adapted meshes based on discretisation error approach for the adaptation criteria $10^{-1}$ (top), $10^{-2}$ (middle), $10^{-3}$ (bottom). 

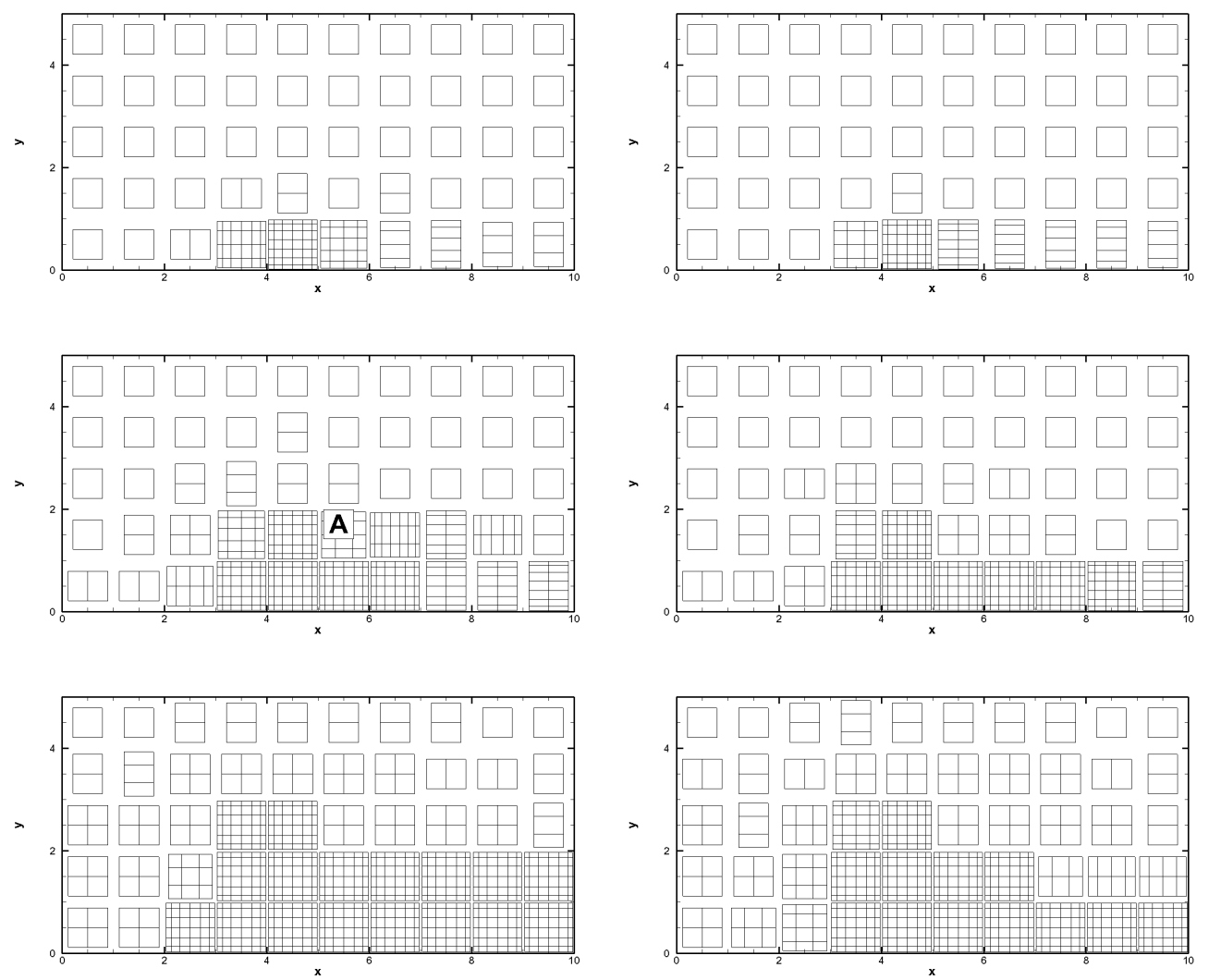

Figure 5.19: Adapted meshes based on the truncation error (left) and the isolated truncation error (right), adaptation criteria $10^{-1}$ (top), 10 ${ }^{-2}$ (middle), $10^{-3}$ (bottom). 


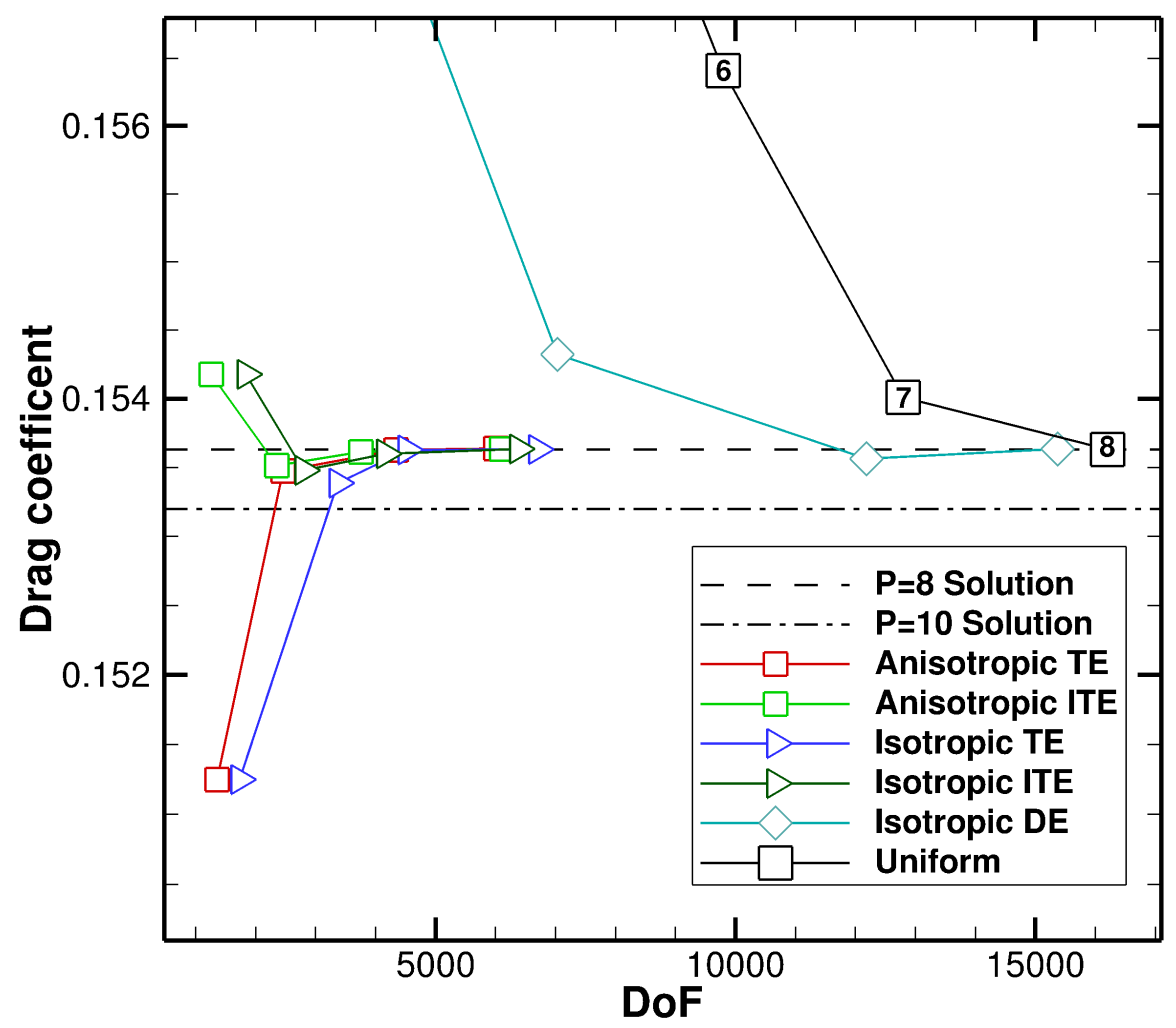

Figure 5.20: Drag value $C_{D}$ and DoF based on different adaptation strategies. Uniform refinement, Discretisation error (DE), Truncation error (TE) and Isolated Truncation error (ITE) strategies are shown. 


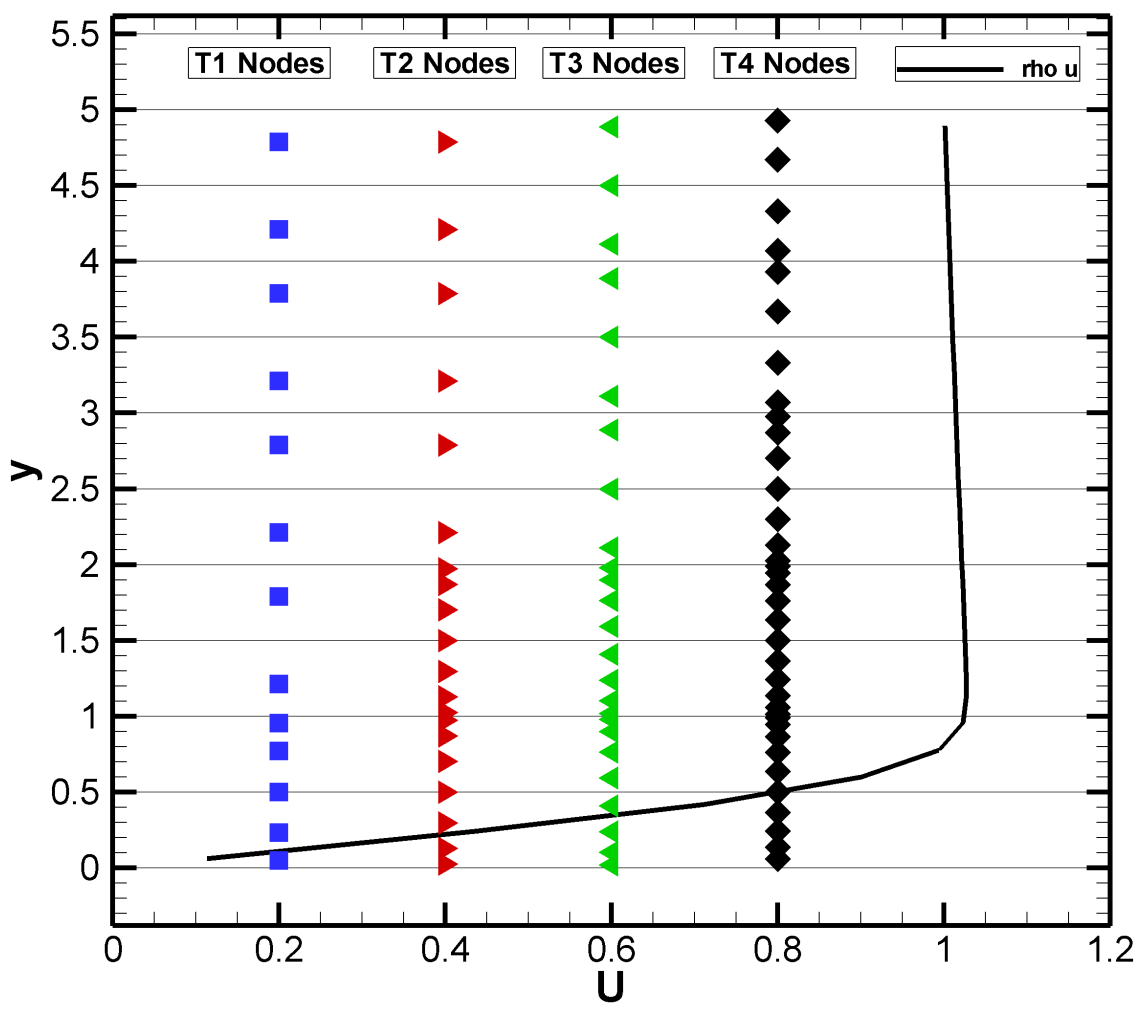

Figure 5.21: Boundary layer profile and node positions for different truncation error thresholds. 

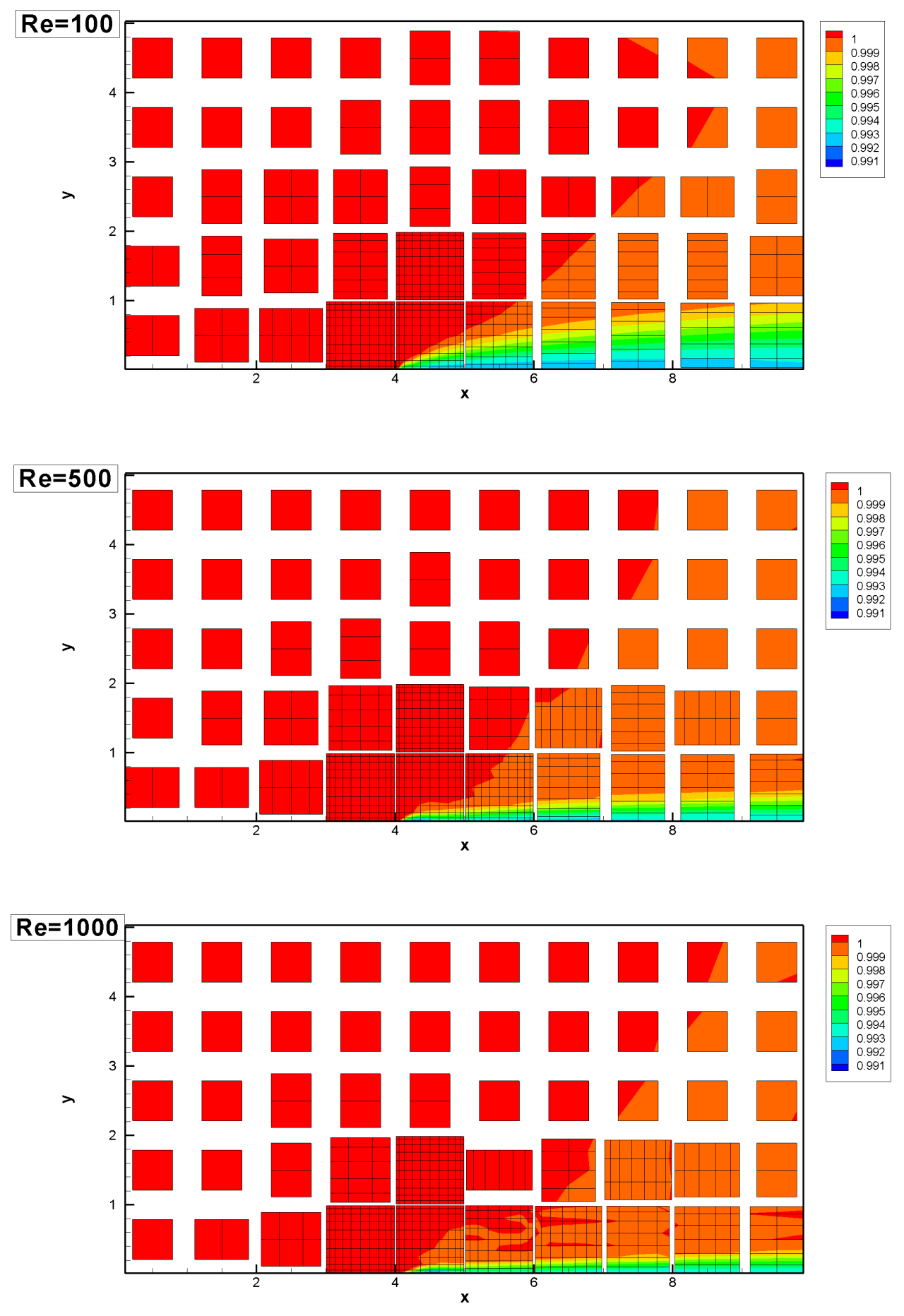

Figure 5.22: Adaptation based on the truncation error and different Reynolds numbers. 

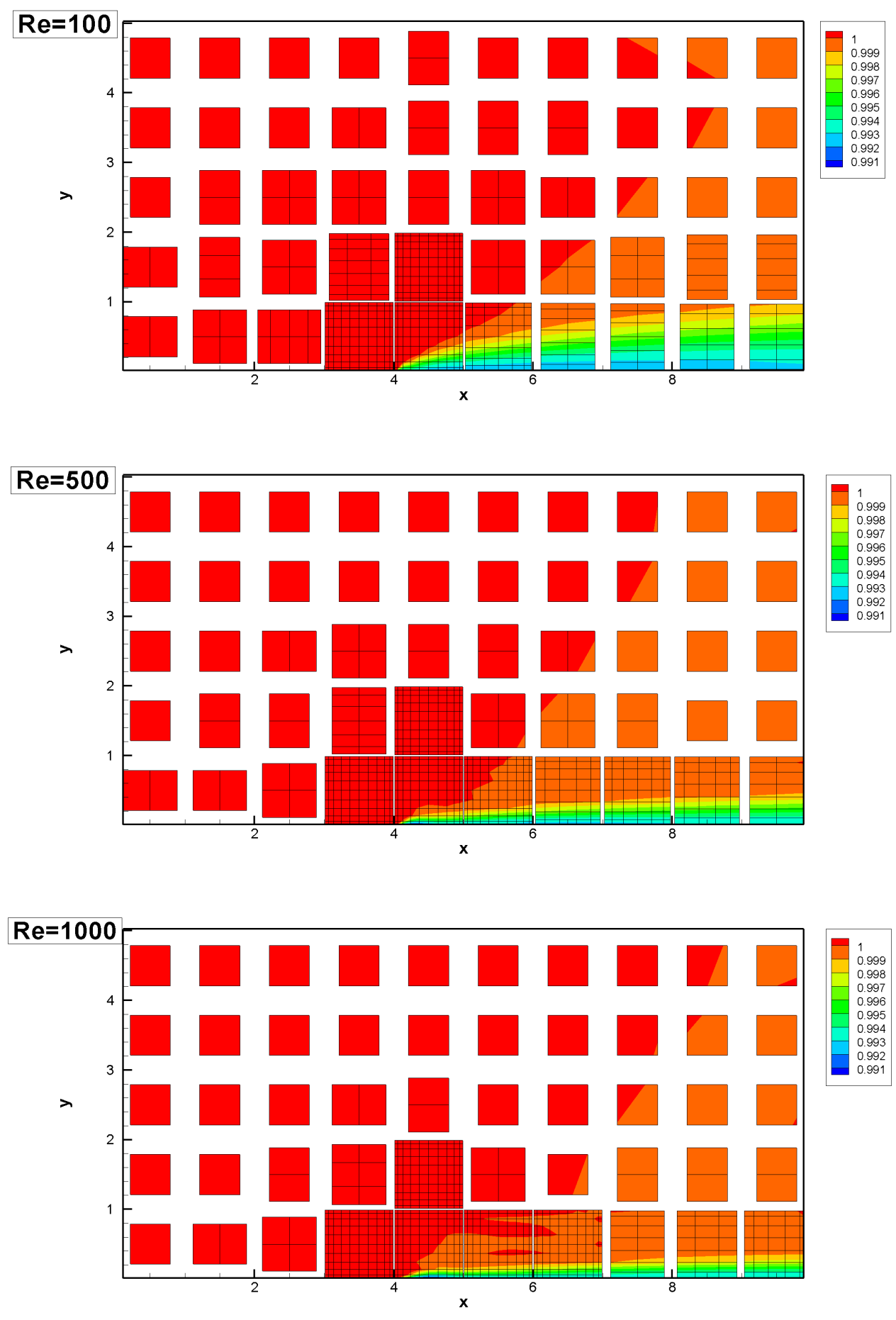

Figure 5.23: Adaptation based on the isolated truncation error and different Reynolds numbers. 


\subsection{Cylinder Test Case}

To further analyse the features of the proposed adaptation algorithms, a Cylinder test case was simulated, using the Navier-Stokes Equations and a Reynolds number of $R e=45$. The previously applied Algorithm 5 will now be used including the proposed two dimensional error extrapolation explained in 4.2.3.

Algorithm 5 showed good results regarding the reduction of degree of freedom for the boundary layer test case. To achieve these results the minimal combination of polynomial orders was chosen that is needed to fulfil the required error threshold. This is equivalent to selecting the minimal local degrees of freedom for the corresponding element. However, the beneficial properties of Algorithm 4 in terms of error extrapolation had not yet been included in the boundary layer test case.

To incorporate this feature, in section 4.2 .3 a two dimensional error extrapolation scheme has already been proposed. This extrapolation enables to enlarge the set of estimates, due to a two dimensional extrapolation, and to select the polynomial order with the minimal degrees of freedoms in the entire set.

To test these features a cylinder test case was simulated using two fine mesh solutions with polynomial order $P=6$ and $P=8$. The properties of the simulation are shown in Table 5.5. For the simulation 254 elements were used and the test case was converged until a residual of Res $=10^{-10}$ was reached. The result of the fine mesh simulation with

\begin{tabular}{|l|l|}
\hline Cylinder diameter & $L_{D}=1$ \\
Elements & $\# E l=264$ \\
Polynomial order & $P=6,8$ \\
Degrees of Freedom & $D o F=7392,9504$ \\
Mach number & $M=0.2$ \\
Reynolds number & $R e=45$ \\
\hline
\end{tabular}

Table 5.5: Cylinder Simulation overview.

polynomial order $P=8$ can be seen in Figure 5.24 for $\rho u$.

To test the functionality of the two dimensional extrapolation, the adaptation results based on the simulation with $P=8$, which does not use extrapolation, see Figure 5.25 (left), are compared to the results based on the $P=6$ results using extrapolation to overcome the missing estimates for $N=6$ and $N=7$ see Figure 5.25, right. The used threshold for the adaptation is $\tau_{\max }=10^{-2}$.

As it can be seen, the results agree very well. Nevertheless minor differences can occur for certain elements, where the extrapolated order is close to the threshold value $\tau_{\max }$, due to the approximate character of the extrapolation. However, an important assumption for this extrapolation is to ensure that the estimates are within the asymptotic range. In the present case this is fulfilled for most of the elements. Nevertheless it is likely that the asymptotic range for low order combinations e.g. $\tau_{8}^{(1,1)}$ may not be reached. Accordingly, for this extrapolation the low order estimates were excluded. 


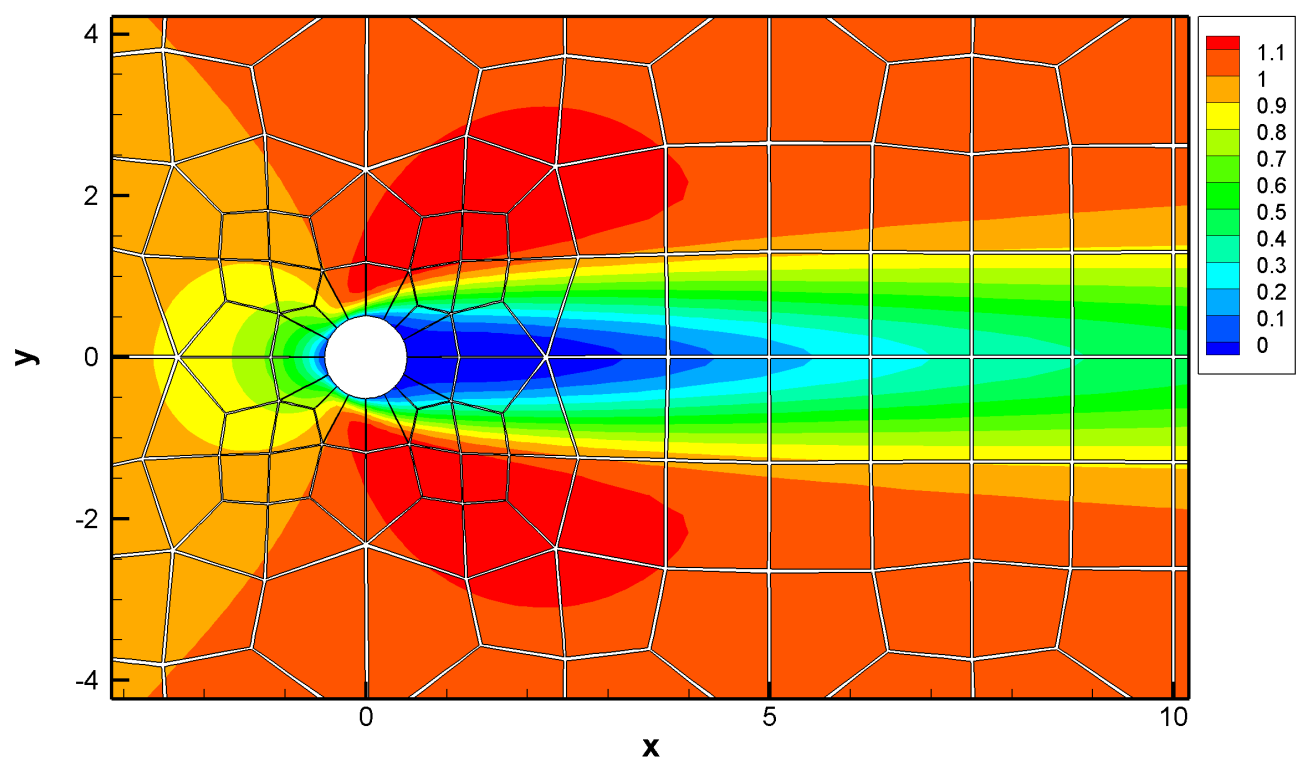

Figure 5.24: Cylinder simulation, $\rho u$ for $P=8$.
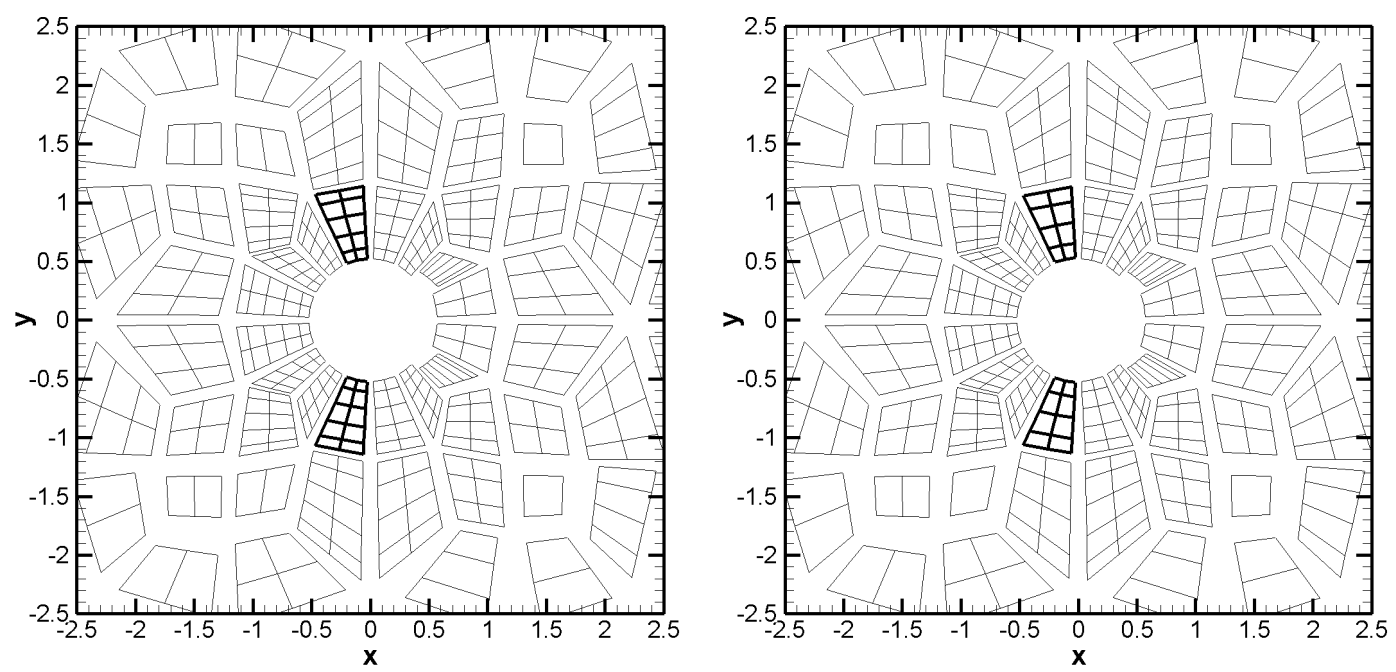

Figure 5.25: Adaptation results based on a converged solution with $P=6$ with extrapolation (left) and based on a solution with $P=8$ with no extrapolation, $\tau_{\max }=10^{-1}$ was used as the adaptation criterion.

Finally applying the Algorithm 5 to the fine solution with $P=8$, using the truncation error as an adaptation criteria and furthermore the two dimensional error extrapolation, leads to the results shown in Figure 5.26. The results for the isolated truncation error can be seen in Figure 5.27. Finally, when the asymptotic range is ensured, the Algorithm 5 with incorporated two dimensional least mean square extrapolation is an efficient approach 
to reduce the degrees of freedom and corresponding cost of the foregoing simulation (in this case: reduction from $P 8$ with 9504 DoF to $P 6$ with 7392). 

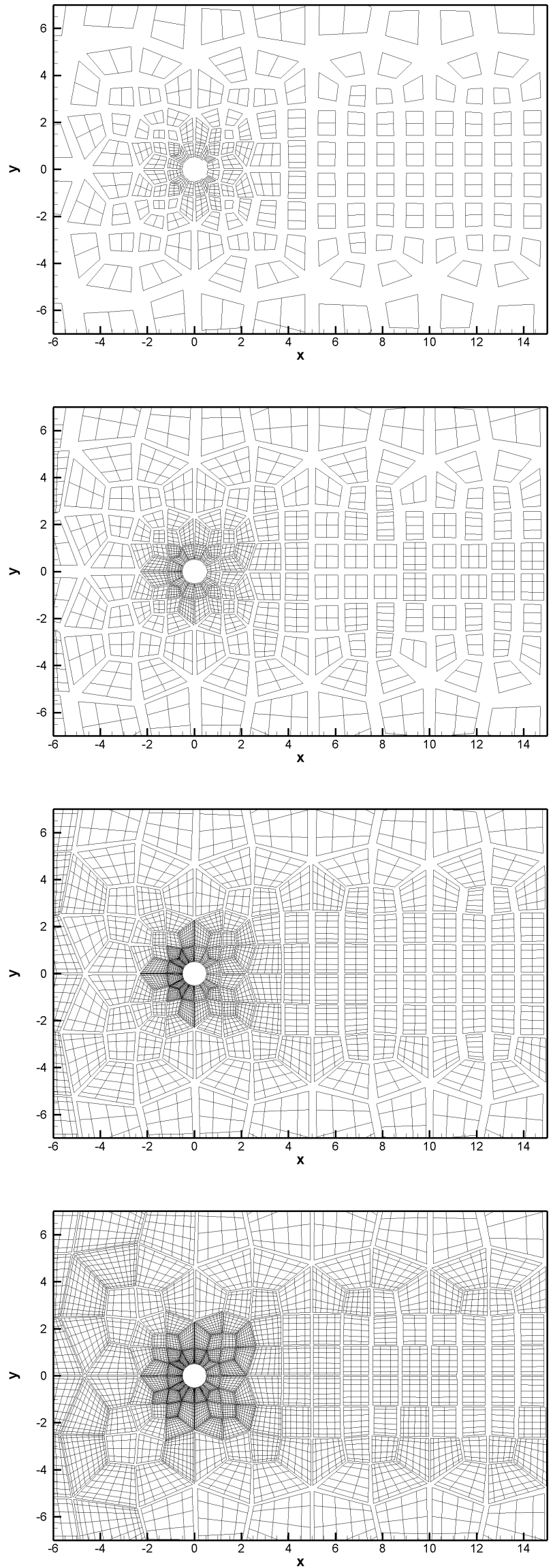

Figure 5.26: Adaptation based on the truncation error and different Reynolds numbers. 

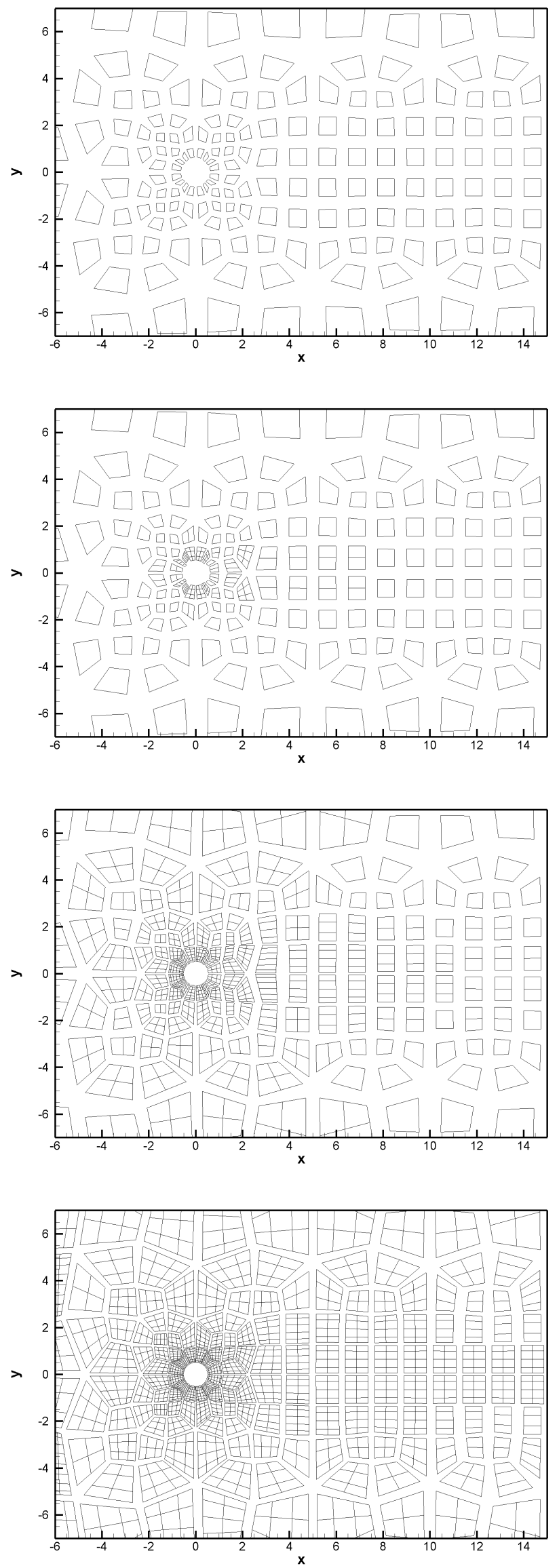

Figure 5.27: Adaptation based on the isolated truncation error and different Reynolds numbers. 


\subsection{Computational Cost}

In this section, the computational cost of the different methods is compared for the boundary layer test case. A truncation error threshold of $\tau_{\max }<10^{-2}$ is defined as the objective. As previously explained, the a posteriori method converges the solution until the maximum residual ( $L_{\infty}$ norm) is below $10^{-10}$ (i.e. pre-adaptation step), then a new p-adapted mesh is computed following Algorithm 2. The solution is subsequently interpolated from the original to the new p-adapted mesh and converged again (i.e. post-adaptation step). In this section, the a posteriori method is tested for a polynomial $P=8$. For the quasi- $a$ priori method results are included following the two approaches with and without correction. If no correction is applied, the solution is converged until a tolerance of $10^{-3}$ is reached (pre-adaptation step), using this solution the truncation error is estimated and a new p-adapted mesh is obtained and converged until $10^{-10}$ (post-adaptation step). Obviously this solution is computationally more efficient than the a posteriori method, since the initial solution is relaxed to a lower tolerance and the rest of the algorithm is equivalent. Finally, the tolerance is set to $10^{-1.5}$, which clearly shortens the computational time, and the correction term is applied afterwards, this is the quasi- $a$ priori corrected method. However, it must be born in mind that the calculation of the correction and the accurate estimation of the truncation error is expensive (i.e. requires the solution of a linear system). The method can, nonetheless, be computationally efficient when considering the overall computational cost, depending on the time required in each step and the time needed to compute the correction factor. In our cases, the Jacobian, $\left.\frac{\partial \mathcal{R}^{P}}{\partial u^{P}}\right|_{u^{P}}$, is stored in sparse format and the solution of the linear system, required by the correction step, is provided by a GMRES iterative solver with block Jacobi preconditioning. Our implementation relies on the PETSc libraries, Balay et al. [Balay 2015], to solve the linear problem in the quasi-a priori corrected method. As a final step, the transfer operator, $\left.\frac{\partial \mathcal{R}^{N}}{\partial u^{N}}\right|_{u^{N}} I_{P}^{N}$, for the iteration error from the fine to the coarse mesh is calculated.

For reference, the adaptation strategies are compared to the computational time of a non-adapted solution with a constant polynomial order $P=10$. This polynomial order is chosen such that it equals the maximum polynomial order that is used in the adaptation algorithm to obtain the required truncation error threshold of $\tau_{\max }<10^{-2}$.

Table 5.6 summarizes the run times of the a posteriori and the two quasi-a priori approaches (with and without correction). These computational costs are relative to the calculation with a homogeneous polynomial $P=10$. It can be seen that the a posteriori already achieves a remarkable speedup of 2.26 times which is exceeded by the speedup of the quasi-a priori method without correction (speedup of 6.59). The most important time gains are provided by the quasi- $a$ priori corrected method, which provides a speedup of 7.61 .

Table 5.7 details the relative amount of time spent by the methods in each part of the algorithm (i.e. pre-adaptation, adaptation and post-adaptation), which are non- 


\begin{tabular}{|l||c|c|}
\hline & Runtime (\% w.r.t. $P=10$ ) & Speedup (w.r.t. $P=10$ ) \\
\hline 0) homogeneous $P=10$ & 100 & 0 \\
I) a posteriori $P=8$ & 44.13 & 2.26 \\
II) quasi- a priori & 15.18 & 6.59 \\
III) quasi- a priori corrected & 13.14 & 7.61 \\
\hline
\end{tabular}

Table 5.6: Runtime and speedup of the a posteriori and quasi-a priori adaptation approaches, non-dimensionalized with respect to a homogeneous polynomial $P=10$ (no adaptation), time convergence until $\|\mathcal{R}(\tilde{u})\|_{L_{\infty}}<10^{-10}$.

dimensionalized with respect to total time of the simulation with homogeneous polynomial $P=10$ (no adaptation). The main differences between the three adaptation strategies can be seen for the pre-adaptation times. Indeed, the quasi- $a$ priori approach requires longer pre-adaptation simulations than the quasi-a priori corrected method $(9.1 / 2.8 \sim 3.3$ times longer). Even though the time to calculate the correction in the adaptation part of the quasi-a priori corrected method is not negligible (3.6), the overall time remains lower (13.14\% of the reference computation as shown in Table 5.6) making this adaptation strategy the most efficient. In addition, it can be seen that the post-adaptation times are similar in the two quasi-a priori approaches. Finally, it should be noted note that the three anisotropic p-adaptation strategies lead to identical adapted meshes as depicted in the previous section Figure 5.15 (bottom).

\begin{tabular}{|l|ccc|}
\hline & Pre adaptation $\%$ & Adaptation $\%$ & Post adaptation \% \\
\hline I) a posteriori $P=8$ & 38.618 & 0.0166 & 5.492 \\
II) quasi- a priori & 9.104 & 0.0182 & 6.059 \\
III) quasi- a priori corrected & 2.830 & 3.5895 & 6.724 \\
\hline
\end{tabular}

Table 5.7: Computational cost of a posteriori and quasi-a priori adaptation algorithms non-dimensionalized with respect to a homogeneous simulation with polynomial $P=10$ (no adaptation).

It can be concluded that the three adaptation strategies reduce the computational cost significantly when compared to a simulation with the same accuracy and with the polynomial order fixed everywhere in the domain. In addition, the two quasi- $a$ priori approaches show significant time reductions, and in particular the quasi- a priori corrected algorithm enables significant speedups.

The advantages of the adaptation process have been summarized in Figure 5.28, where the accuracy and computational cost are depicted for all the computed cases. These include the uniform polynomial meshes (non-adapted) and the adapted meshes using the three adaptation strategies. The figure also gives the degrees of freedom (DoF) used for each simulation. The outperforming results of the adaptation strategies over the uniform polynomial are clear. Furthermore, for the same accuracy the quasi- $a$ priori methods (with and without correction) show cost improvements over the a posteriori technique. Finally, the quasi- $a$ priori corrected method shows the best performance among all the 
proposed techniques.

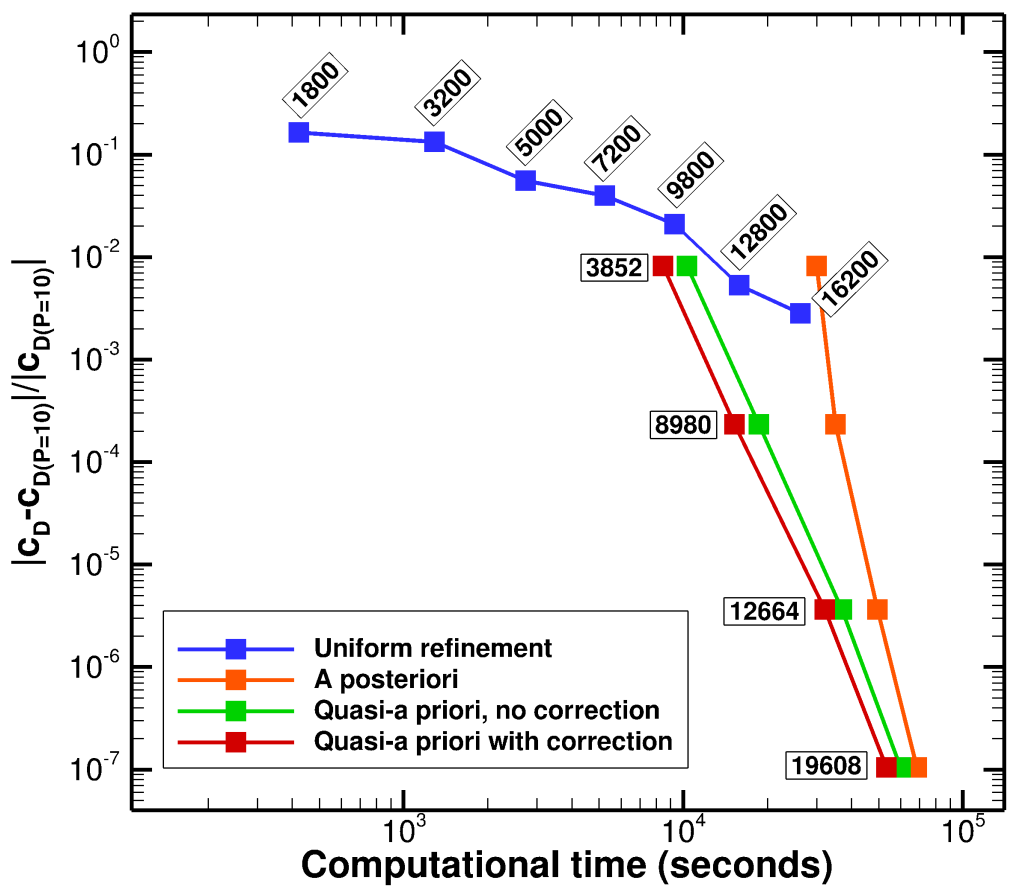

Figure 5.28: Relative error in drag coefficient $\frac{\left|c_{D}-c_{D(P=10)}\right|}{\left|c_{D(P=10)}\right|}$ and computational time (in seconds). The reference drag $c_{D(P=10)}$ is calculated on a uniformly refined mesh with $P=10$. The blue line shows uniform polynomial order (non-adapted meshes). Adapted simulations include: a posteriori approach (orange), quasi- a priori approach without correction (green) and quasi- a priori corrected (red). The number of DoF for each simulation is shown in the boxes. 


\section{Conclusions and Outlook}

\subsection{Conclusions}

Different novel isotropic and anisotropic p-adaptation strategies have been presented in this work. The truncation error estimation has been successfully used to select the elements and directions that require adaptation. Indeed, the direct relation of the truncation error to the numerical error makes it an excellent criterion for mesh refinement.

Algorithms based on an a posteriori estimation and quasi-a priori approach have been presented and have shown to provide faster converged solutions than when a uniformly high polynomial order is selected. The quasi- $a$ priori techniques enable an accurate and reliable adaptation method based on not fully time-converged solutions. It has been shown that the incorporation of a correction term to the non-converged solution significantly improves the error estimate by cancelling out the first order iteration error.

Furthermore different error criteria have been used to perform the local p-adaptation and were compared in terms of accuracy, efficiency and degrees of freedom. The regions for adaptation (refinement or coarsening) are selected via three different procedures. A discretisation error based adaptation, that quantifies the decay of the energy associated with the different modes is compared to two variants of truncation error approaches. The first truncation error approach relies on the estimation of the truncation error based on all terms appearing in the discrete discontinuous Galerkin variational formulation, whilst the second (the isolated truncation error) only takes the volume terms into account.

These adaptation strategies are compared with each other for an inviscid symmetric NACA0012 airfoil and a viscous flat plate boundary layer. The results show that truncation error based strategies require fewer degrees of freedom (approximately $80 \%$ less than uniform refinement), to attain similar accuracy, than the discretisation error (approximately $40 \%$ less than uniform refinement) approach (i.e. discretisation error based method).

Finally, anisotropic approaches, that lead to adaptation results with different polynomial orders in different coordinate directions, can furthermore reduce the degrees of freedom and result in a refinement solution that properly reflects the flow features. Anisotropic adaptation results based on the truncation error outperform isotropic versions (approximately $15 \%$ less DoF than isotropic versions) specially when considering flows with preferential directions such as boundary layers.

Remarkable savings in computational cost are achieved based on the proposed anisotropic 
adaptation algorithms. In particular, the calculations using the quasi- $a$ priori approach with correction term enables a speedup of 7.6 when compared to the non-adapted solution.

\subsection{Outlook}

The presented algorithm and adaptation strategies show very good properties in terms of accuracy, computational cost and degrees of freedom. They enable highly accurate results while keeping the computational cost and the degrees of freedom low.

The truncation error adaptation method still has considerable potential for further advancements, that may lead to even more effective schemes and help to make Spectral Element Method more applicable for large scale industrial simulations.

Valuable insights have been given in this work in terms of anisotropic p-refinement. Further improvement can be made when h-refinement strategies are incorporated in the proposed methods. In particular, in areas where the asymptotic range of convergence is not achieved, an adaptation method that uses h-refinement (or a combination of h- and p-refinement) may lead to significant improvements of the results.

To the current state, automatic mesh adaptation for high order discontinuous Galerkin methods is predominantly done with adjoint based approaches. Methods based on adjoints achieve very accurate results. Nevertheless they also result in very high computational costs. Further studies have to be done to compare these type of methods to the proposed approaches based on the truncation error. Also a combination of both techniques has been proposed for low order methods [Ponsin 2015] and needs to be investigated for high order methods in the future. 


\section{Validation and Scaling}

\section{Contents}

A.1 Comparison of Compressible and Incompressible Solver . . . . . . . . . . . 97

A.2 Numerical Experiments . . . . . . . . . . . . . . . . . . . . . . 98

A.2.1 Laminar Boundary Layer on a Flat Plate . . . . . . . . . . . . . 99

A.2.2 Flow over a NACA0012 and NACA0015 Profile . . . . . . . . . 100

The following section deals with the validation and scaling of the presented solver. In the first part the limitations of the compressible solver will be studied when running it in incompressible scenarios. For this purpose the compressible code has been compared to another solver that uses the incompressible formulation. Furthermore the scalability of the Discontinuous Galerkin Spectral Element method is shown. The comparison and scaling test encompass two test-cases of the 2nd international workshop on high order methods May 27 - 28, 2013 hold in Cologne, Germany: "C1.3 Flow over the NACA0012 airfoil" and "C1.4 Flat plate Boundary Layer", see [Wang 2013] for a summary of the test cases.

\section{A.1 Comparison of Compressible and Incompressible Solver}

The introduced compressible solver will be compared to the incompressible solver of Ferrer [Ferrer 2012a]. Both high order DG formulations share the favourable characteristics of minimising spatial numerical errors and hence enable comparisons of flow formulations. In addition, both codes use the non-dimensional form of the NS equations. The purpose of this chapter is to provide a qualitative and quantitative comparison to assess the limits of accuracy of compressible solvers when computing low Reynolds number and low Mach number flows.

In particular, drag coefficients and boundary layer profiles (e.g. displacement thickness) are compared with theoretical estimates when available (e.g. Blasius solution).

Compressible Solver The previously introduced nodal, explicit 2D high order DG code based on the DGSEM formulation [Kopriva 2009] has been used to provide results for compressible flows. To increase the flexibility of the solver a mortar element method is used to couple the element faces if hanging nodes or elements with varying polynomial orders are used in the computational mesh. 
Temporal advancement is provided by a 3rd Order Runge Kutta scheme. The code uses OpenMP parallelization with shared memory.

Incompressible Solver Flow solutions of the 3D incompressible NS equations are obtained from the unsteady high order $h / p$ DG - Fourier solver developed by Ferrer and detailed in [Ferrer 2011], [Ferrer 2012b] and [Ferrer 2012a]. This high order solver provides highly accurate solutions on static and moving domains composed of mixed triangularquadrilateral meshes and can cope with curved boundary elements. A mixed implicitexplicit second order stiffly stable method is used to discretise the NS equations in time whilst spatial discretization is provided by the DG - Symmetric Interior Penalty Galerkin formulation with modal basis functions in the $x-y$ plane. Spatial discretization in the $z$-direction is provided by a purely spectral method that uses Fourier series and allows computation of spanwise periodic three-dimensional flows. The code is parallelized for distributed memory clusters using a combination of MPI and OpenMP paradigms.

The solver has been widely validated for a variety of flows, including bluff body flows, airfoil and blade aerodynamics under static and rotating conditions [Ferrer 2012b] [Ferrer 2012a].

In this paper all computations performed using the incompressible solver are limited to $2 \mathrm{D}$.

\section{A.2 Numerical Experiments}

Two test cases will be presented: Flow over the NACA0012 airfoil and Flat Plate Boundary Layer. In addition to the flow conditions specified by the workshop, lower Reynolds numbers and various low Mach numbers are included to study the differences between compressible and incompressible formulations. A summary of the various cases is provided in Table A.1. The calculations presented in this work have been performed on a 64

\begin{tabular}{|l||c|c|c|}
\hline Case & Reynolds number & Mach & AOA \\
\hline \hline Boundary Layer Compressible solver & $60,600,3600$ & $0.05,0.1,0.3$ & - \\
Boundary Layer Incompressible solver & 60,600 & - & - \\
\hline NACA0012 Compressible solver & 5000 & 0.5 & $1^{\circ}$ \\
\hline NACA0015 Compressible solver & 100 & $0.2,0.3$ & $0^{\circ}$ \\
NACA0015 Incompressible solver & 100 & - & $0^{\circ}$ \\
\hline
\end{tabular}

Table A.1: Summary of test cases detailing Reynolds number, Mach number and Angle of Attack (AOA).

Beowulf cluster based on 8 quad-core Intel Xeon processor with nominal speed of 2.40 GHz. Taubench is running in 10.265 seconds in one core. The authors follow the workshop requirement to calculate the converged solutions. Both, compressible and incompressible solution are started from a uniform free stream and are advanced in time until a steady 
state solution is reached.

\section{A.2.1 Laminar Boundary Layer on a Flat Plate}

For the Flat Plate Boundary Layer Simulation a domain of $L_{X}=10$ and $L_{Y}=5$ is used while the plate has a length of $L_{P}=6$ and is preceded by a symmetry region of length $L_{H}=4$. The current unstructured grid for the compressible solver is shown in Figure A.1 (a). The grid for the compressible solver was generated by the in house software SpecMesh2D and Gambit [Inc. 1998] was used to generate the meshes for the incompressible solver, see Figure A.1 (b).

Figure A.1 (a) shows streamwise velocity contours obtained by the compressible solver on a quadrilateral grid and A.1 (b) shows the streamwise velocity contours obtained using the incompressible solver with a tetrahedral grid. The polynomial order for the compressible solver was set to $\mathrm{k}=8$ in all elements in $\mathrm{x}$ and $\mathrm{y}$ direction, whilst $\mathrm{k}=11$ was used for the incompressible calculations.

The calculations of the compressible solver were accomplished for different Mach numbers and compared to the incompressible and the Blasius solution, see Schlichting [Schlichting 2000]. Table A.2 summarizes the $c_{d}$ values computed with both solvers together with Blasius analytical estimates: $c_{d}=\frac{1.328}{\sqrt{R e_{L_{p}}}}$. The polynomial order for the compressible solver was set to $k=8$ in all elements in $x$ and $y$ direction, whilst $k=11$ was used for the incompressible calculations. Furthermore, the error

$$
\text { Error }_{c_{d}}=\left|\frac{c_{d_{\text {Compressible }}}-c_{d_{\text {Incompressible }}}}{c_{d_{\text {Incompressible }}}}\right| \times 100
$$

is displayed in the table. In addition, the boundary layer thicknesses $\delta_{1}^{R e_{L}}=\int_{0}^{\infty}\left(1-\frac{U}{U_{\infty}}\right) d y$ are compared.

When comparing the drag coefficient at the lowest Reynolds number (i.e. $R e_{L_{p}}=60$ ), the table shows that incompressible and compressible solutions using the lowest Mach number provide similar results, $1.4 \%$ error. However, the time step needed to achieve converged solutions using the compressible solver are 100-times smaller (i.e. longer computation). At $R e_{L_{p}}=600$ all computations show similar errors when compared to the incompressible results. The discrepancies between computed results and boundary layer drag coefficient obtained from the Blasius solution may be explained by the singularity at the plate leading edge, which is not taken into account by the Blasius theory, Schlichting [Schlichting 2000]. Comparing the boundary layer thickness, it can be seen that the incompressible solver provides more accurate results than the compressible solver. Furthermore, it can be recognized that the compressible solution with the lowest Mach number is the most accurate one of the compressible results.

Figure A.3 shows the drag error for different polynomial orders and the respective work units for two grids and their respective degrees of freedom. 


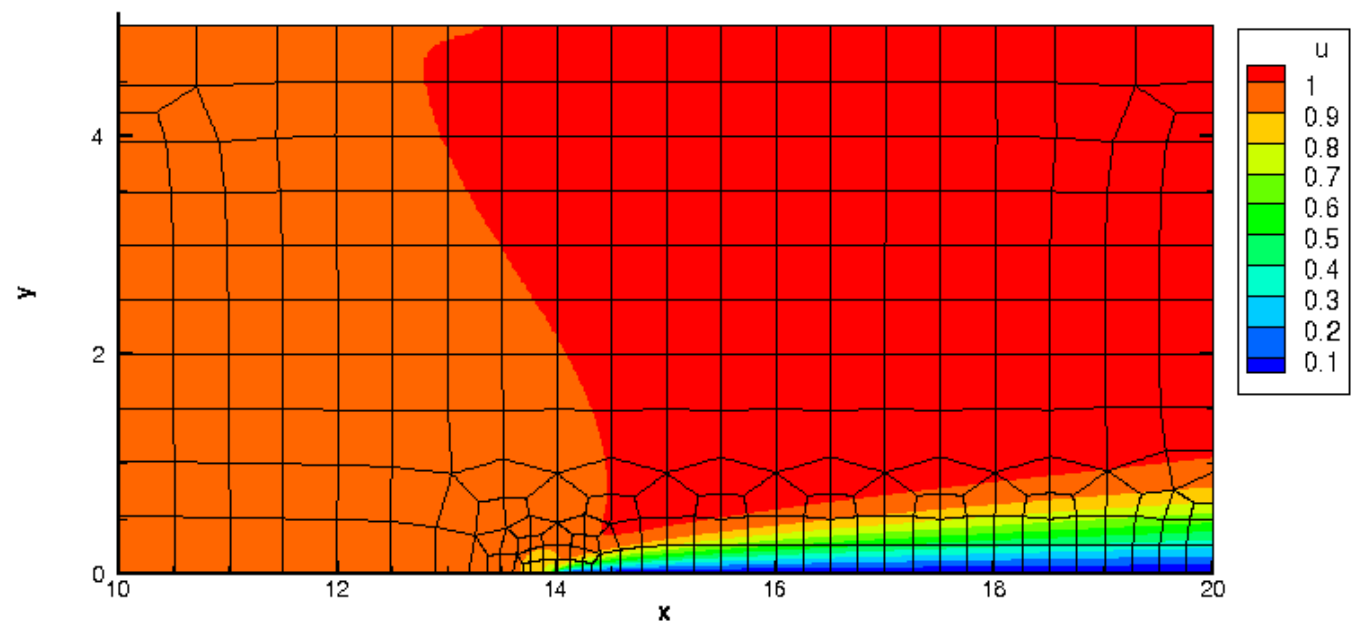

(a) Flowfield of streamwise velocity using the compressible solver; quadrilateral grid; polynomial order $\mathrm{k}=8$.

u

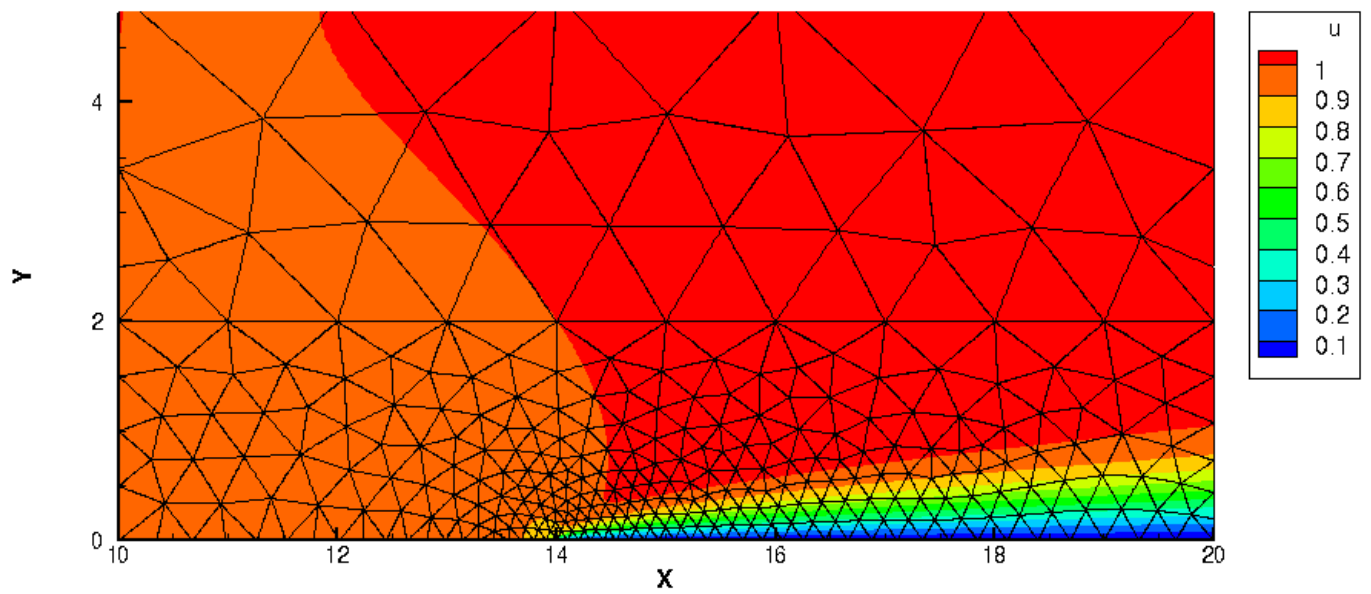

(b) Flowfield of streamwise velocity using the incompressible solver; tetrahedral grid; polynomial order $\mathrm{k}=11$.

Figure A.1: Comparison of compressible and incompressible solvers on a Boundary Layer; streamwise-velocity for $R e_{L_{p}}=600$.

\section{A.2.2 Flow over a NACA0012 and NACA0015 Profile}

Figure A.4 shows examples of the grids used for compressible and incompressible formulations for a NACA0015. The left triangular mesh is used by the incompressible solver whilst the quadrilateral mesh, on the right, is used by the compressible code. The calculations for the NACA0012 are performed in similar grids.

A.2.2.0.1 NACA0012 Figure A.5 shows the result of the NACA0012 obtained by the compressible solver at $R e_{c}=5000, \mathrm{Ma}=0.5$ and $\alpha=1^{\circ}$, as defined in the workshop. 


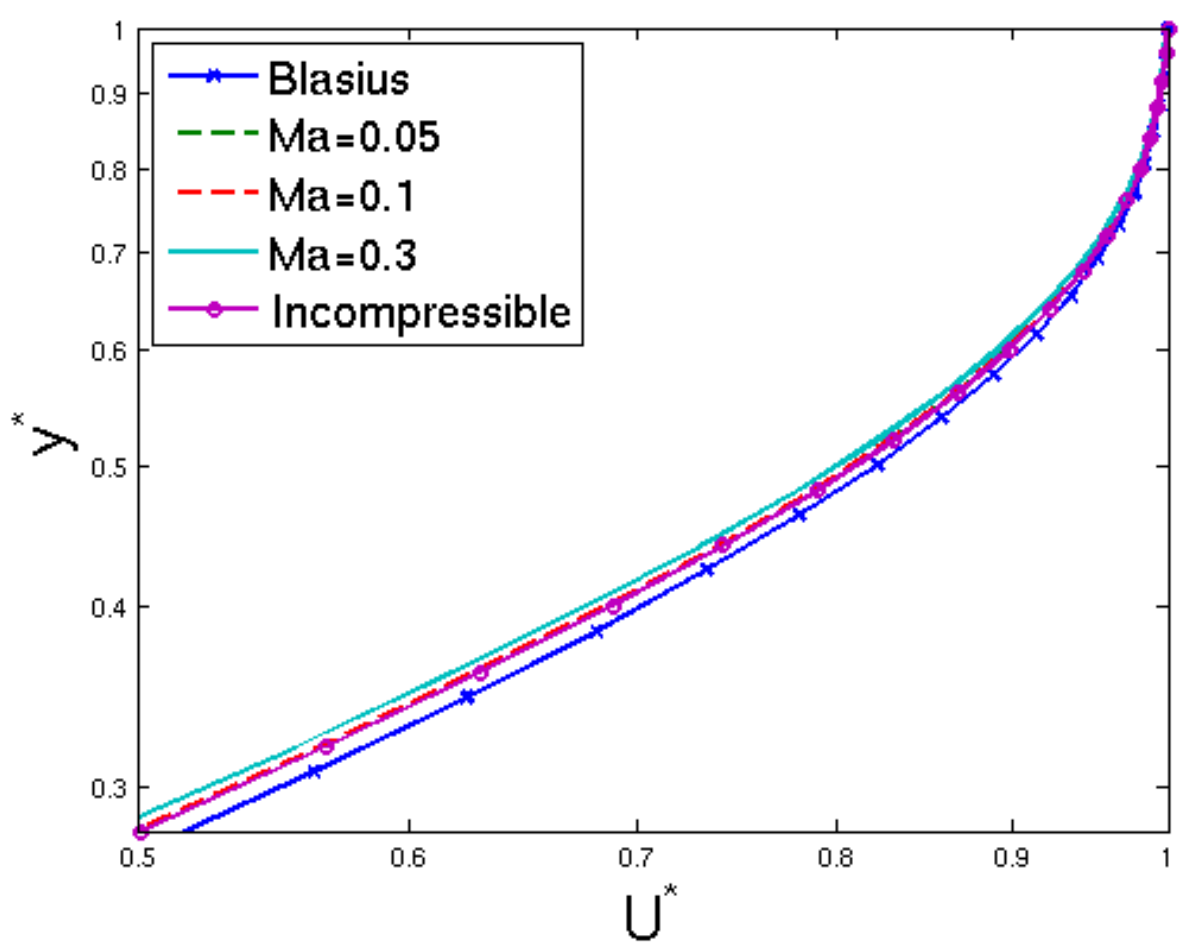

Figure A.2: Velocity profiles at a distance of $L=5.2$ downstream of the leading edge at a Reynolds number of $\operatorname{Re}_{L}=520$.

\begin{tabular}{|l||l|l|l|l|l|l|}
\hline \multicolumn{1}{|c||}{} & \multicolumn{3}{c|}{$\operatorname{Re}_{L_{p}}=60$} & \multicolumn{5}{c|}{$\operatorname{Re}_{L_{p}}=600$} \\
\hline & $c_{d}$ & error $_{c_{d}}[\%]$ & $c_{d}$ & error $_{c_{d}}[\%]$ & $\delta_{1}$ & error $_{\delta_{1}}[\%]$ \\
\hline Incompressible & 0.22266 & - & 0.06039 & - & 0.3097 & - \\
\hline $\mathrm{Ma}=0.05$ & 0.21934 & 1.49 & 0.05967 & 1.20 & 0.3105 & 0.28 \\
\hline $\mathrm{Ma}=0.1$ & 0.23031 & 3.43 & 0.05956 & 1.38 & 0.3113 & 0.51 \\
\hline $\mathrm{Ma}=0.3$ & 0.26267 & 17.97 & 0.05978 & 1.01 & 0.3164 & 2.18 \\
\hline Blasius & 0.17144 & 23.00 & 0.05421 & 10.23 & 0.3022 & 2.42 \\
\hline
\end{tabular}

Table A.2: Viscous drag-coefficients $c_{D_{v}}$ at $\operatorname{Re} R e_{L_{p}}=60$ and $R e_{L_{p}}=600$ for compressible, incompressible and Blasius solution. In addition, results for the displacement thickness $\delta_{1}^{R_{e_{L}}=520}$ are summarized.

As expected, p-refinement, Figure A.5 (a) shows an exponential scaling for higher degrees of freedom in terms of accuracy and work units. When performing h-refinement, as shown in Figure A.5 (b) , for a polynomial order $\mathrm{k}=6$, a slope of 7.25 can be seen, confirming that the order of accuracy $h^{p+1}$, where $\mathrm{h}$ defines the mesh size and $\mathrm{k}$ the polynomial order, is achieved.

Additional comparisons between the compressible and the incompressible solvers have been performed for the NACA0015 airfoil at $R e_{c}=100$ and zero Angle of Attack $\alpha=$ $0^{\circ}$ for two Mach numbers $(\mathrm{Ma}=0.2,0.3)$. Compressible solutions have been obtained using a polynomial order $\mathrm{k}=8$, whilst incompressible results are obtained with $\mathrm{k}=6$. 


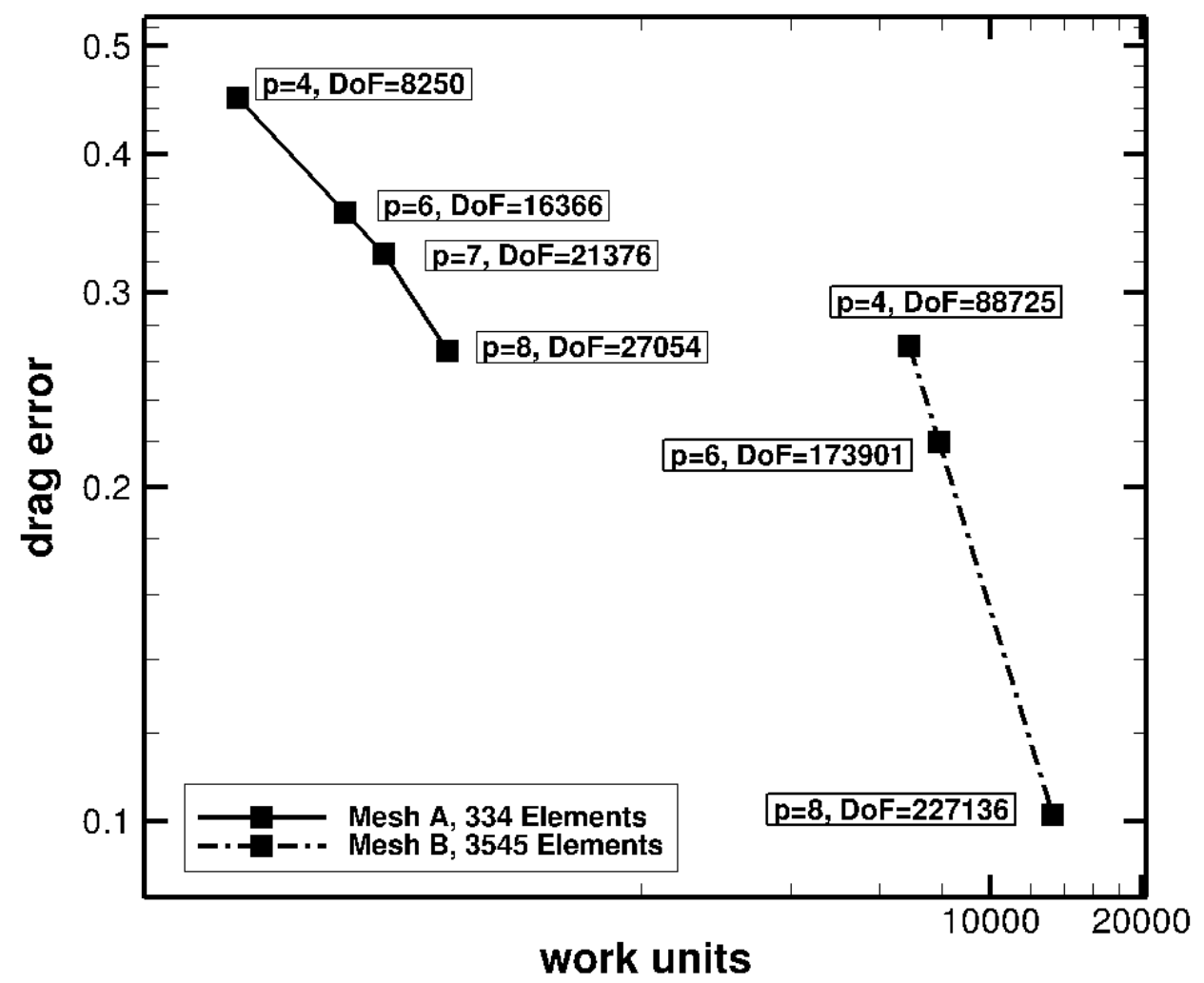

Figure A.3: Drag error for the flat plate at $R e_{L_{p}}=3600$.

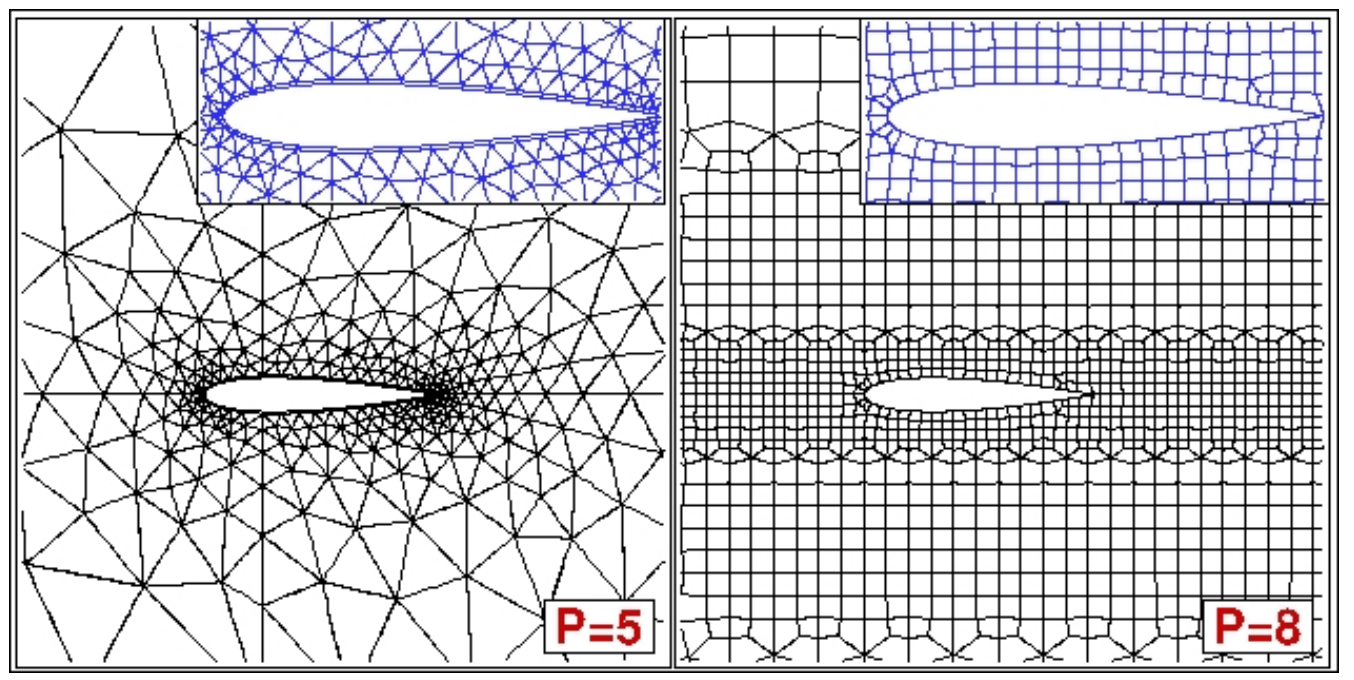

Figure A.4: NACA0015 airfoil meshes using triangles for the incompressible and quadrilaterals for the compressible solver.

These results are summarized in Table A.3. The table shows that even when low Mach numbers are considered, there is a considerable difference between the compressible and 
incompressible results for both viscous and pressure components of the drag coefficient. In addition, these differences increase when increasing the Mach number. For completeness, a comparison of the pressure and skin friction coefficients is included in Figure A.6. It can be seen that both codes agree very well overall. Minor differences are seen near the leading edge for the skin friction plot, which may be due to lack of local spatial resolution. This difference is still under investigation.

\begin{tabular}{|l||c|c|c|c|c|c|}
\hline & $c_{d}$ & error $_{c_{d}}[\%]$ & $c_{d}$ & error $_{D_{p}}[\%]$ & error $_{D_{v}}[\%]$ & error $_{c_{D}}[\%]$ \\
\hline \hline Incompressible & 0.2044 & 0.3402 & 0.5446 & - & - & - \\
\hline $\mathrm{Ma}=0.2$ & 0.2104 & 0.3538 & 0.5642 & 2.90 & 4.00 & 3.59 \\
\hline $\mathrm{Ma}=0.3$ & 0.2192 & 0.3691 & 0.5883 & 7.22 & 8.51 & 8.02 \\
\hline
\end{tabular}

Table A.3: NACA0015 at $R e_{c}=100$ and $\alpha=0^{\circ}$. Drag-coefficients $c_{D}=c_{D_{p}}+c_{D_{v}}$, including pressure $c_{D_{p}}$ and viscous $c_{D_{v}}$ components, for the compressible and incompressible solvers.

It was shown that the compressible results approach the incompressible solutions as the Reynolds number increases. For low Reynolds number, the Mach number is required to decrease in order for both solvers to provide similar results in lift and drag coefficients. In addition, it was shown that a compressible solver, when running under incompressible flow conditions (i.e. using low Mach numbers without corrections or preconditioning), does not provide the same aerodynamic quantities as the incompressible solver. 


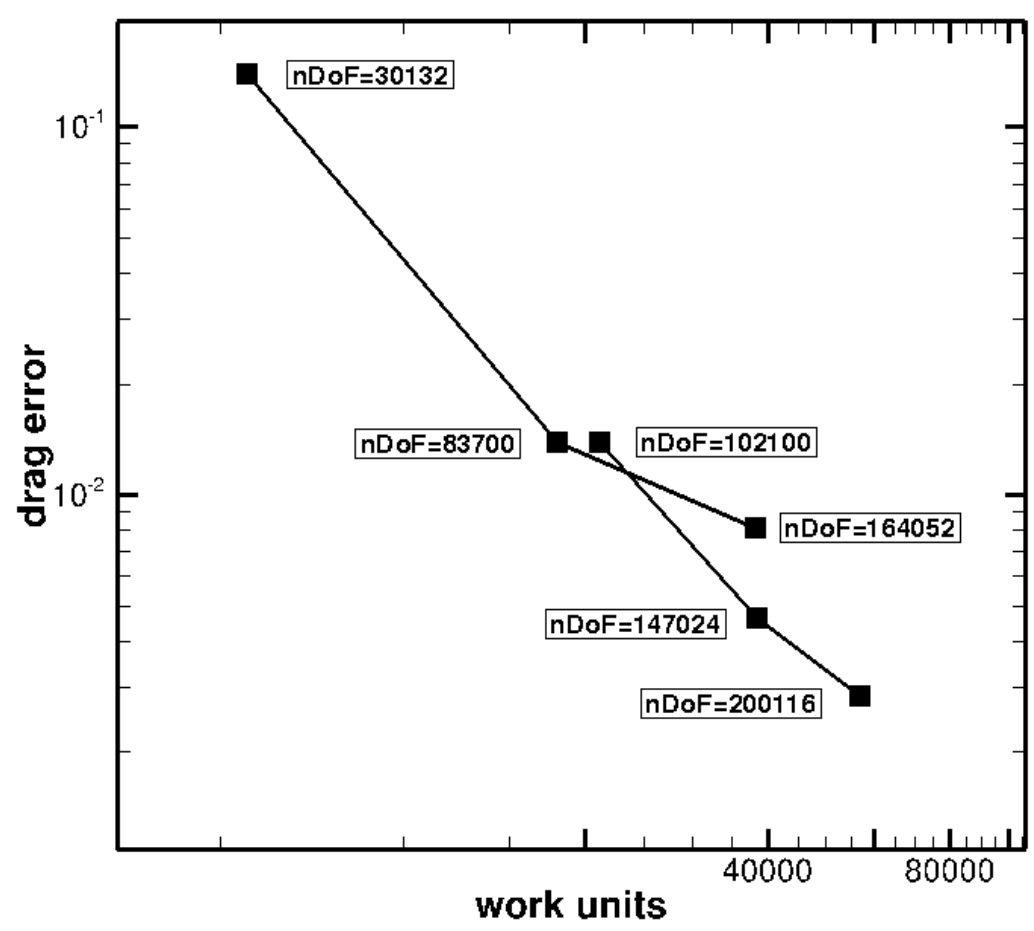

(a) p-refinement

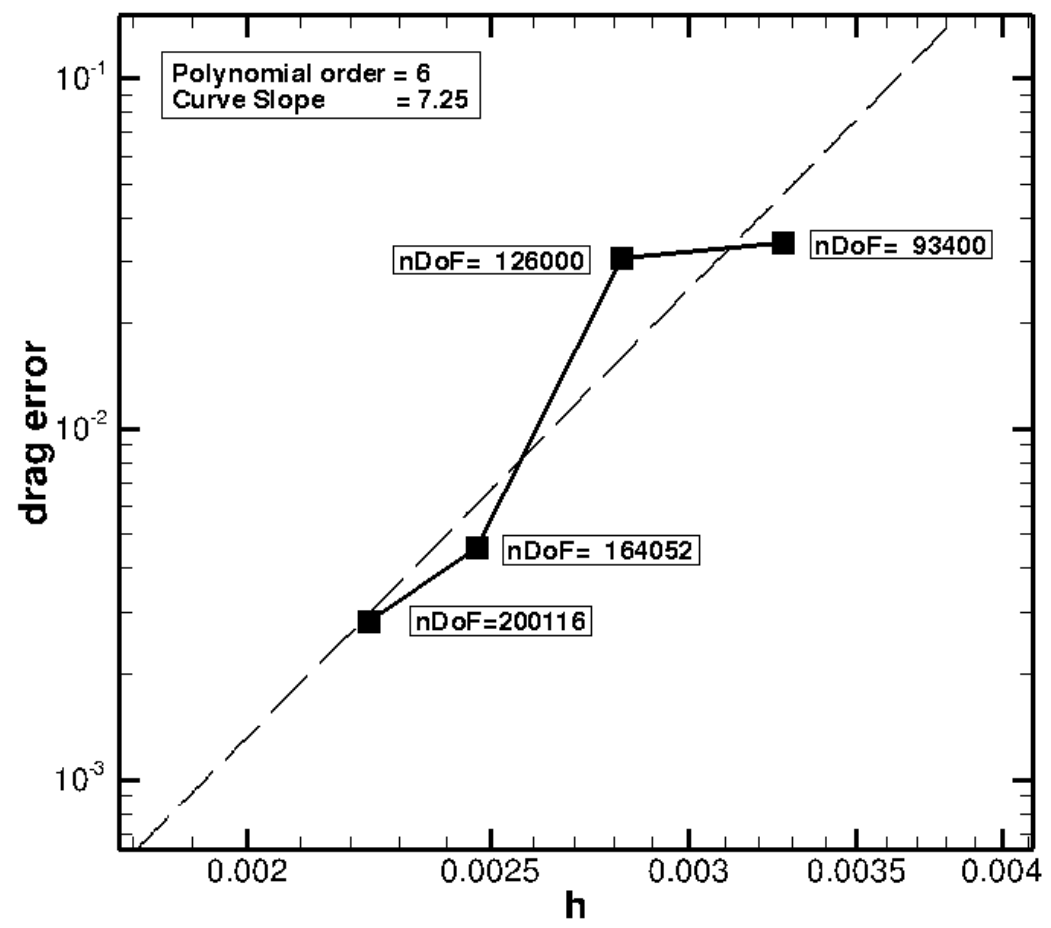

(b) h-refinement

Figure A.5: h/p refinement for NACA0012. 

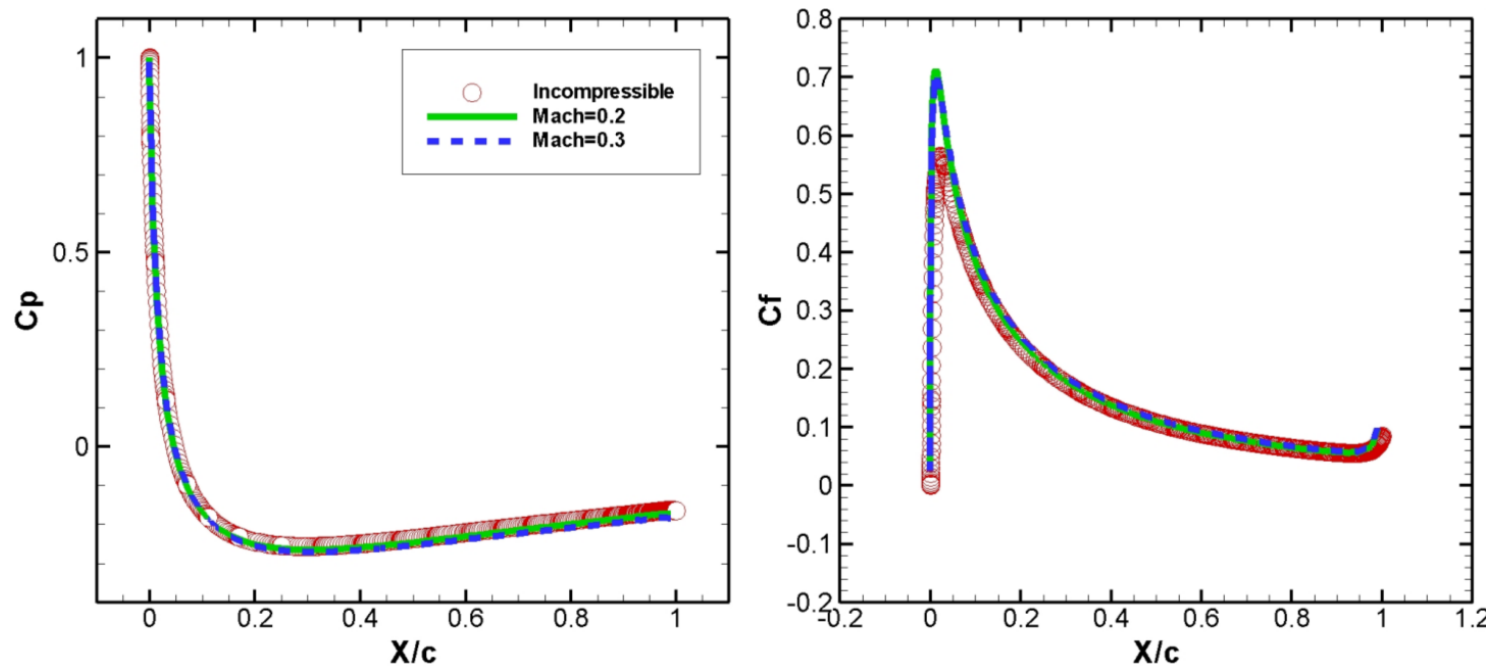

Figure A.6: Pressure (Cp) and skin friction (Cf) coefficients for a NACA0015 at $\mathrm{Re}=100$ using compressible and incompressible solvers. 



\section{Bibliography}

[Ainsworth 2007] M. Ainsworth. A posteriori error estimation for discontinuous Galerkin finite element approximation. SIAM Journal on Numerical Analysis, vol. 45, no. 4, pages 1777-1798, 2007. (Cited on page 2.)

[Arnold 2001] D.N. Arnold, F. Brezzi, B. Cockburn and L.D. Marini. Unified analysis of discontinuous Galerkin methods for elliptic problems. SIAM Journal of Numerical Analysis, vol. 39, no. 5, pages 1749-1779, 2001. (Cited on page 72.)

[Babuska 1981] I. Babuska. Dorr, M.R.: Error Estimates for the Combined $h$ and $p$ Versions of the Finite Element Method. Numerische Mathematik, vol. 37, pages 257-278, 1981. (Cited on page 49.)

[Balasubramanian 2009] R. Balasubramanian and J. C. Newman. Adjoint-based error estimation and grid adaptation for functional outputs: Application to two-dimensional, inviscid, incompressible flows. Computers \& Fluids, vol. 38, no. 2, pages 320 - 332, 2009. (Cited on page 3.)

[Balay 2015] S. Balay, S. Abhyankar, M. F. Adams, J. Brown, P. Brune, K. Buschelman, L. Dalcin, V. Eijkhout, W. D. Gropp, D. Kaushik, M. Knepley, L. C. McInnes, K. Rupp, B. F. Smith, S. Zampini and H. Zhang. PETSc Users Manual. Rapport technique ANL-95/11 - Revision 3.6, Argonne National Laboratory, 2015. (Cited on page 92.)

[Barosan 2006] I. Barosan, P.D. Anderson and H.E.H. Meijer. Application of mortar elements to diffuse-interface methods. Computers and Fluids, vol. 35, no. 10, pages 1384-1399, 2006. (Cited on page 49.)

[Bassi 1997] F. Bassi and S. Rebay. A high-order accurate discontinuous Finite Element method for the numerical solution of the compressible Navier-Stokes equations. Journal of Computational Physics, vol. 131(2), 1997. (Cited on page 1.)

[Bassi 2006] F. Bassi, A. Crivellini, D. A. Di Pietro and S. Rebay. An artificial compressibility flux for the discontinuous Galerkin solution of the incompressible NavierStokes equations. Journal of Computational Physics, vol. 218, no. 2, pages $794-$ 815, 2006. (Cited on page 1.)

[Baumann 1997] C. E. Baumann. An hp-adaptive discontinuous finite element method for computational fluid dynamics. PhD thesis, University of Texas at Austin, 1997. (Cited on page 1.) 
[Berger 1984] M. J. Berger and J. Oliger. Adaptive mesh refinement for hyperbolic partial differential equations. Journal of Computational Physics, vol. 53, no. 3, pages 484 - 512, 1984. (Cited on page 49.)

[Berger 1987] M. J. Berger. Adaptive finite difference methods in fluid dynamics. Courant Institute of Mathematical Sciences New York University, 1987. (Cited on page 4.)

[Bernert 1997] K. Bernert. $\tau$-Extrapolation-Theoretical foundation, numerical experiment, and application to Navier-Stokes equations. SIAM Journal on Scientific Computing, vol. 18, no. 2, pages 460-478, 1997. (Cited on pages 3 and 4.)

[Boyd 1989] J. P. Boyd. Chebyshev and fourier spectral methods. Springer, 1989. (Cited on pages 9,11 and 13.)

[Brandt 2011] A. Brandt and O. E. Livne. Multigrid Techniques. Society for Industrial and Applied Mathematics, 2011. (Cited on page 3.)

[Canuto 2006] C. Canuto, M. Y. Hussaini, A. Quarteroni and T. A. Zang. Spectral Methods. Fundamentals in Single Domains. Springer, Berlin, 2006. (Cited on pages 9, $14,17,18,19$ and 25.)

[Canuto 2010] C. G. Canuto, Y. Hussaini, A. Quarteroni and T. A. Zang. Spectral methods: Fundamentals in single domains. Scientific Computation. Springer, 2010. (Cited on pages 27, 29, 34 and 47.)

[Cockburn 1998] B. Cockburn and C. W. Shu. The local discontinuous Galerkin method for time dependent convection-diffusion systems. SIAM Journal of Numerical Analysis, vol. 35, pages 2440-2463, 1998. (Cited on page 1.)

[Cockburn 2009] B. Cockburn, G. Kanschat and D. Schötzau. An Equal-Order DG Method for the Incompressible Navier-Stokes Equations. Journal of Scientific Computing, vol. 40, no. 1-3, pages 188-210, 2009. (Cited on page 1.)

[Derlaga 2015] J. M. Derlaga, T. Phillips, C. J. Roy and J. Borggaard. Adjoint and truncation error based adaptation for finite volume schemes with error estimates. In 53rd AIAA Aerospace Sciences Meeting, AIAA SciTech. American Institute of Aeronautics and Astronautics, January 2015. (Cited on page 3.)

[Dwight 2008] R. P. Dwight. Heuristic a Posteriori Estimation of Error Due to Dissipation in Finite Volume Schemes and Application to Mesh Adaptation. Journal of Computational Physics, vol. 227, no. 5, pages 2845-2863, 2008. (Cited on page 2.)

[Ferrer 2011] E. Ferrer and R.H.J. Willden. A high order discontinuous Galerkin Finite Element solver for the incompressible Navier-Stokes equations. Computers \& Fluids, vol. 46, no. 1, pages 224-230, 2011. (Cited on pages 1 and 98.) 
[Ferrer 2012a] E. Ferrer. A high order Discontinuous Galerkin-Fourier incompressible 3D Navier-Stokes solver with rotating sliding meshes for simulating cross-flow turbines. PhD thesis, University of Oxford, 2012. (Cited on pages 2, 97 and 98.)

[Ferrer 2012b] E. Ferrer and R.H.J. Willden. A high order discontinuous Galerkin Fourier incompressible 3D Navier-Stokes solver with rotating sliding meshes. Journal of Computational Physics, vol. 231, no. 21, pages 7037-7056, 2012. (Cited on pages 1 and 98.)

[Ferrer 2014] E. Ferrer, D. Moxey, R.H.J. Willden and S. Sherwin. Stability of projection methods for incompressible flows using high order pressure-velocity pairs of same degree: Continuous and Discontinuous Galerkin formulations. Communications in Computational Physics, vol. 16, no. 3, pages 817-840, 2014. (Cited on page 1.)

[Fraysse 2012a] F. Fraysse, J. de Vicente and E. Valero. The estimation of truncation error by $\tau$-estimation revisited. Journal of Computational Physics, vol. 231, no. 9, pages 3457-3482, 2012. (Cited on pages 3, 4 and 39.)

[Fraysse 2012b] F. Fraysse, E. Valero and J. Ponsin. Comparison of Mesh Adaptation Using the Adjoint Methodology and Truncation Error Estimates. AIAA Journal, 2012. (Cited on page 3.)

[Fraysse 2013] F. Fraysse, E. Valero and G. Rubio. Quasi-a priori truncation error estimation and higher order extrapolation for non-linear partial differential equations. Journal of Computational Physics, vol. 253, no. 0, pages 389 - 404, 2013. (Cited on pages 4 and 5 .)

[Fraysse 2014] F. Fraysse, G. Rubio, J. de Vicente and E. Valero. Quasi-a priori mesh adaptation and extrapolation to higher order using tau-estimation. Aerospace Science and Technology, vol. 38, no. 0, pages $76-87,2014$. (Cited on page 3.)

[Fulton 2003] S. R. Fulton. On the accuracy of multigrid truncation error estimates. Electronic Transactions on Numerical Analysis, vol. 15, pages 29-37, 2003. (Cited on page 4.)

[Gao 2011] H. Gao and Z. J. Wang. A residual-based procedure for hp-adaptation on 2D hybrid meshes. AIAA Paper, vol. 492, 2011. (Cited on page 3.)

[Georgoulis 2003] E. H. Georgoulis. Discontinuous Galerkin methods on shape-regular and anisotropic meshes. PhD thesis, University of Oxford D. Phil. Thesis, 2003. (Cited on page 34 .)

[Hartmann 2006] R. Hartmann. Error estimation and adjoint-based adaptation in aerodynamics. In P. Wesseling, E. Onate and J. Périaux, editeurs, Proceedings of the ECCOMAS CFD 2006, September 5-8, Egmond aan Zee, The Netherlands, 2006. (Cited on page 3.) 
[Henderson 1994] R. Henderson. Unstructured spectral element methods: Parallel algorithms and simulations (Ph. D. Thesis). 1994. (Cited on page 49.)

[Hesthaven 2008] J. S. Hesthaven and T. Warburton. Nodal discontinuous Galerkin methods: algorithms, analysis, and applications. Springer Science \& Business Media, 2008. (Cited on pages 27, 34 and 47.)

[Hindenlang 2012] F. Hindenlang, G. J. Gassner, C. Altmann, A. Beck, M. Staudenmaier and C.-D. Munz. Explicit discontinuous Galerkin methods for unsteady problems. Computers \& Fluids, vol. 61, pages 86 - 93, 2012. (Cited on pages 56 and 57.)

[Inc. 1998] Fluent Ansys Inc. Gambit Users guide. Lebanon NH, 1998. (Cited on page 99.)

[Karniadakis 1999] G. Karniadakis and S. J. Sherwin. Spectral/hp Element Methods for CFD. Numerical mathematics and scientific computation. Oxford University Press, 1999. (Cited on page 2.)

[Karniadakis 2013] G. Karniadakis and S. Sherwin. Spectral/hp element methods for computational fluid dynamics. Oxford University Press, 2013. (Cited on page 27.)

[Kompenhans 2016] M. Kompenhans, G. Rubio, E. Ferrer and E. Valero. Adaptation strategies for high order discontinuous Galerkin methods based on Tau-estimation. Journal of Computational Physics, vol. 306, pages 216 - 236, 2016. (Cited on page 44.)

[Kopriva 2009] D. A. Kopriva. Implementing Spectral Methods for Partial Differential Equations: Algorithms for Scientists and Engineers. Springer, 1st édition, 2009. (Cited on pages 1, 9, 14, 16, 27, 29, 30 and 97.)

[Lancaster 1986] P. Lancaster and K. Salkauskas. Curve and surface fitting: an introduction. Computational mathematics and applications. Academic Press, 1986. (Cited on page 53.)

[Landmann 2008] B. Landmann, M. Kessler, S. Wagner and E. Krämer. A parallel highorder discontinuous Galerkin code for laminar and turbulent flows. Computers \& Fluids, vol. 37, no. 4, pages $427-438,2008$. (Cited on page 1.)

[Lawson 1995] C. Lawson and R. Hanson. Solving least squares problems. Society for Industrial and Applied Mathematics, 1995. (Cited on page 53.)

[Mavriplis 1990] C. Mavriplis. A Posteriori Error Estimators for Adaptive Spectral Element Techniques. In Pieter Wesseling, editeur, Proceedings of the Eighth GAMMConference on Numerical Methods in Fluid Mechanics, volume 29 of Notes on $\mathrm{Nu}$ merical Fluid Mechanics (NNFM), pages 333-342. Vieweg+Teubner Verlag, 1990. (Cited on page 49.) 
[Mavriplis 1994] C. Mavriplis. Adaptive mesh strategies for the spectral element method. Computer Methods in Applied Mechanics and Engineering, vol. 116, no. 14, pages 77 - 86, 1994. (Cited on page 2.)

[Nguyen 2007] N. C. Nguyen, P. O. Persson and J. Peraire. RANS solutions using high order discontinuous Galerkin methods. 45th AIAA Aerospace Science Meeting and Exhibit, Reno, Nevada, 2007. (Cited on page 1.)

[Oliver 2007] T. A. Oliver and D. L. Darmofal. An Unsteady Adaptation Algorithm for Discontinuous Galerkin Discretizations of the RANS Equations. 18th AIAA Computational Fluid Dynamics Conference, Reno, Nevada, 2007. (Cited on page 1.)

[Peiro 2005] J. Peiro and S. J. Sherwin. Finite Difference, Finite Element and Finite Volume Methods for Partial Differential Equations. In Sidney Yip, editeur, Handbook of Materials Modeling, pages 2415-2446. Springer Netherlands, 2005. (Cited on page 1.)

[Persson 2006] P. Persson and J. Peraire. Sub-cell shock capturing for discontinuous Galerkin methods. AIAA Aerospace Sciences Meeting and Exhibit, vol. 44th, 2006. (Cited on page 48.)

[Phillips 2011] T. Phillips and C. Roy. Residual methods for discretization error estimation. 20th AIAA Computational Fluid Dynamics Conference, 2011. (Cited on page 3.)

[Phillips 2014] T. Phillips. Residual-based Discretization Error Estimation for Computational Fluid Dynamics Residual-based Discretization Error Estimation for Computational Fluid Dynamics. PhD thesis, Virginia Polytechnic Institute and State University, 2014. (Cited on page 3.)

[Ponsin 2015] J. Ponsin, F. Fraysse, M. Gomez and M. Cordero-Gracia. An adjointtruncation error based approach for goal-oriented mesh adaptation. Aerospace Science and Technology, vol. 41, no. 0, pages 229 - 240, 2015. (Cited on page 96.)

[Reed 1973] H. Reed and T. R. Hill. Triangular mesh methods for the neutron transport equation. Rapport technique LA-UR-73-479, Los Alamos Scientific Laboratory, 1973. (Cited on pages 1 and 27.)

[Riviere 2008] B. Riviere. Discontinuous Galerkin methods for solving elliptic and parabolic equations: theory and implementation. Society for Industrial and Applied Mathematics, Philadelphia, PA, USA, 2008. (Cited on page 1.)

[Roache 1998] P. J. Roache. Verification and validation in computational science and engineering. Hermosa, Albuquerque, 1998. (Cited on page 61.) 
[Roy 2002] C. J. Roy, T. M. Smith and C. C. Ober. Verification of a compressible CFD code using the method of manufactured solutions. In 32nd AIAA Fluid Dynamics Conference, 2002. (Cited on page 60.)

[Roy 2009] C. J. Roy. Strategies for driving mesh adaptation in CFD, AIAA 2009-1302, 47th AIAA Aerospace Sciences Meeting, Orlando, Florida, January 5-8, 2009. 2009. (Cited on page 2.)

[Roy 2010] C. J. Roy. Review of discretization error estimators in scientific computing. AIAA Paper, vol. 126, page 2010, 2010. (Cited on pages 3 and 36.)

[Rubio 2013] G. Rubio, F. Fraysse, J. de Vicente and E. Valero. The estimation of truncation error by $\tau$-estimation for Chebyshev spectral collocation method. Journal of Scientific Computing, vol. 57, no. 1, pages 146-173, 2013. (Cited on pages 4 and 39.)

[Rubio 2014] G. Rubio, F. Fraysse, D. A. Kopriva and E. Valero. Quasi-a priori truncation error estimation in the DGSEM. Journal of Scientific Computing, pages 1-31, 2014. (Cited on pages 4, 5, 34, 39, 40, 41, 44, 47 and 71.)

[Rubio 2015] G. Rubio. Truncation error estimation in the Discontinuous Galerkin Spectral Element Method. PhD thesis, Technical University Madrid, 2015. (Cited on pages $9,14,17,18,31,32,36,37,57$ and 58 .)

[Schlichting 2000] H. Schlichting and K. Gersten. Boundary-layer theory. Springer - Originally published by McGraw Hill - New York - 1979, 2000. (Cited on page 99.)

[Shahbazi 2007] K. Shahbazi, P. F. Fischer and C. R. Ethier. A high-order discontinuous Galerkin method for the unsteady incompressible Navier-Stokes equations. Journal of Computational Physics, vol. 222, no. 1, pages 391 -407, 2007. (Cited on page 1.)

[Shih 2009] T. I. P. Shih and B. R. Williams. Development and evaluation of an a posteriori method for estimating and correcting grid-induced errors in solutions of the Navier-Stokes equations. AIAA Paper, vol. 1499, page 2009, 2009. (Cited on page 3.)

[Syrakos 2006] A. Syrakos and A. Goulas. Finite volume adaptive solutions using SIMPLE as smoother. International Journal for Numerical Methods in Fluids, vol. 52, no. 11, pages 1215-1245, 2006. (Cited on page 4.)

[Syrakos 2012] A. Syrakos, G. Efthimiou, J. G. Bartzis and A. Goulas. Numerical experiments on the efficiency of local grid refinement based on truncation error estimates. Journal of Computational Physics, vol. 231, no. 20, pages 6725 - 6753, 2012. (Cited on page 3.) 
[Toro 1997] E. F. Toro. Riemann solvers and numerical methods for fluid dynamics: a practical introduction. Springer, Berlin, New York, 1997. (Cited on page 29.)

[van der Vegt 2002] J. J. W. van der Vegt and H. van der Ven. Space time discontinuous galerkin finite element method with dynamic grid motion for inviscid compressible flows: I. general formulation. Journal of Computational Physics, vol. 182, no. 2, pages $546-585,2002$. (Cited on page 2.)

[Wang 2013] Z.J. Wang, K. Fidkowski, R. Abgrall, F. Bassi, D. Caraeni, A. Cary, H. Deconinck, R. Hartmann, K. Hillewaert, H.T. Huynh, N. Kroll, G. May, P.O Persson, B. van Leer and M. Visbal. High-order CFD methods: current status and perspective. International Journal for Numerical Methods in Fluids, vol. 72, no. 8, pages 811-845, 2013. (Cited on pages 1, 71 and 97.)

[Zhang 2005] M. Zhang and C.-W. Shu. An analysis of and a comparison between the discontinuous Galerkin and the spectral finite volume methods. Computers \& Fluids, vol. 34, pages 581-592, 2005. (Cited on page 36.) 
